\title{
Diversity relations of
}

plants and soil mierobes

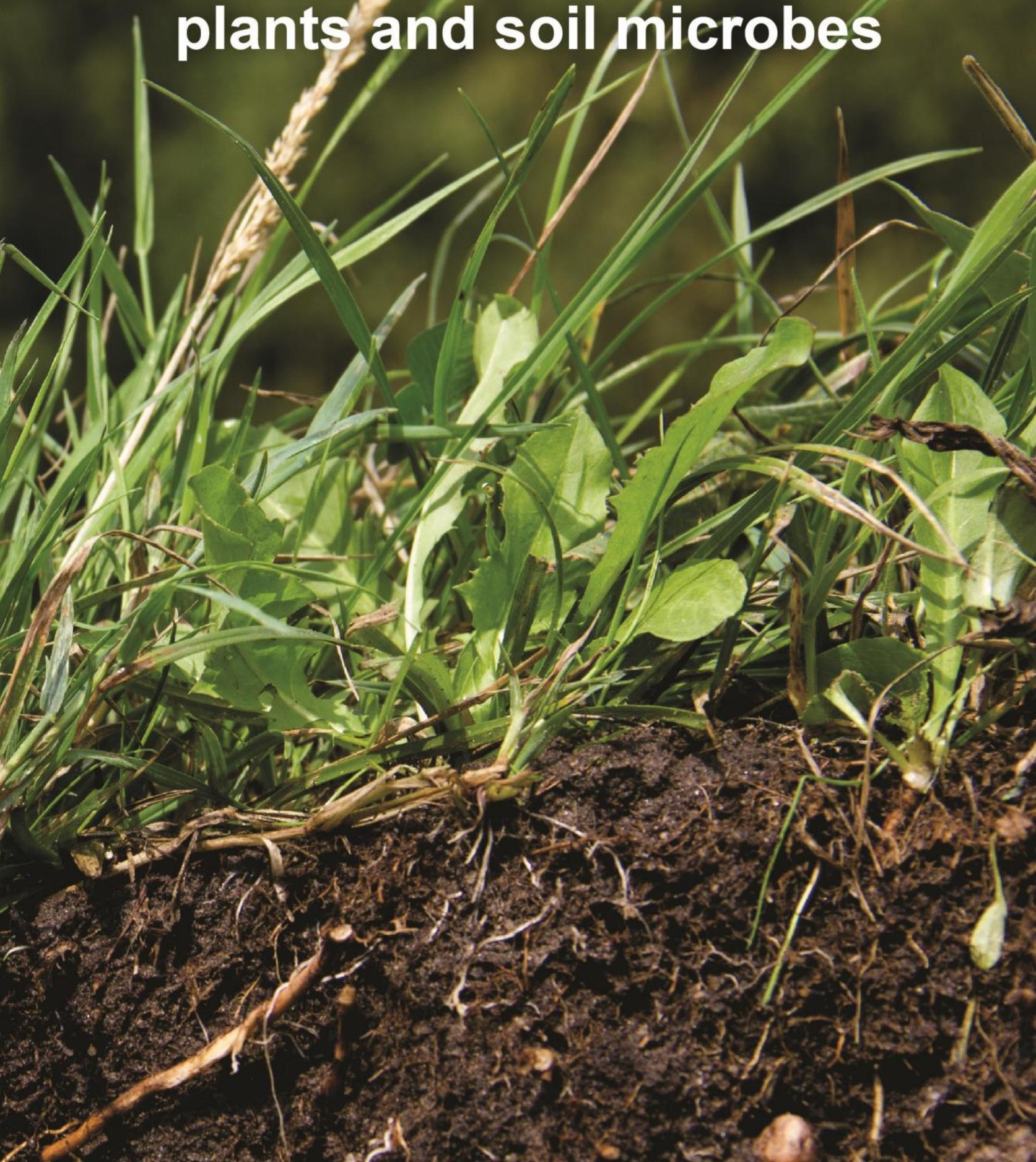





\section{Diversity relations of \\ plants and soil microbes}

Sigrid Dassen 


\section{Thesis committee}

\section{Promotors}

Prof. Dr G.B. De Deyn

Personal chair at the Soil Biology Group

Wageningen University \& Research

Prof. Dr W.H. van der Putten

Special Professor Functional Biodiversity

Wageningen University \& Research and Netherlands Institute of Ecology (NIOO-KNAW),

Wageningen

\section{Co-promotor}

Prof. Dr G.A. Kowalchuk

Professor of Ecology and Biodiversity

Utrecht University

\section{Other members}

Prof. Dr J. Falcao Salles, University of Groningen

Dr E. Morriën, University of Amsterdam

Prof. Dr L. Poorter, Wageningen University \& Research

Dr J. Weedon, VU Amsterdam

This research was conducted under the auspices of the C.T. de Wit Graduate School for

Production Ecology \& Resource Conservation (PE\&RC). 


\title{
Diversity relations of
}

\section{plants and soil microbes}

\author{
Sigrid Dassen
}

\section{Thesis}

submitted in fulfilment of the requirements for the degree of doctor at Wageningen University

by the authority of the Rector Magnificus,

Prof. Dr A.P.J. Mol, in the presence of the

Thesis Committee appointed by the Academic Board

to be defended in public

on Monday 29 October 2018

at 4 p.m. in the Aula. 
Sigrid Dassen

Diversity relations of plants and soil microbes

166 pages

PhD thesis, Wageningen University, Wageningen, the Netherlands (2018)

With references, with summary in English and Dutch

ISBN: 978-94-6343-341-9

DOI: https://doi.org/10.18174/458498 




\section{Table of contents}

Chapter $1 \quad$ General introduction

Chapter 2 Differential responses of soil bacteria, fungi, archaea and protists to plant species richness and plant functional group identity

Chapter 3 Plant traits drive root-associated fungal community composition more than plant species richness

Chapter 4 Plant trait effects on richness and abundance in the soil food web 73

Chapter 5 Mycorrhizal networks in restoration grasslands suppress seedling 97 biomass

Chapter $6 \quad$ General discussion

References

Summary / Samenvatting

Acknowledgements / Dankwoord

About the author

List of publications 


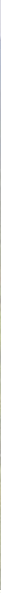

The Jena Main Experiment. 
Chapter 1

General introduction 


\section{Chapter 1}

\section{Plant diversity and ecosystem functions}

Humans depend on natural resources for survival and well-being (Millennium Ecosystem Assessment 2005; Cardinale et al. 2012). Yet, at the same time we put tremendous pressure on our environment by means of agricultural intensification (eutrophication, changed hydrology, intensified land management), climate change, and industrial pollution. As a consequence, plant species richness is declining worldwide and across all biomes (Sala et al. 2000; Grime 2002; Kier et al. 2005; Georg Niedrist et al. 2009). Preservation of plant species richness is essential for ecosystem functioning (Meyer et al. 2016; Isbell et al. 2017), as well as for maintenance of species richness and abundance of vertebrates, invertebrates, and microbes (Hooper et al. 2005). Moreover, plant cover protects the soil from erosion, improves water infiltration, increases soil carbon storage, and reduces loss of nutrients. Numerous studies have shown that, under the same environmental conditions, soils with relatively high plant species richness are better in fulfilling these functions than soils with relatively low numbers of plant species (Meyer et al. 2016; Isbell et al. 2017).

Results from early research on plant biodiversity effects on ecosystem functions pointed toward a positive relation between plant species richness and plant biomass production (Naeem et al. 1994; Tilman et al. 1996, 2001; Hector 1999). To date, these findings generate debates, and new biodiversity experiments have been initiated to unravel the underlying mechanisms (Loreau et al. 2001; Díaz et al. 2003; Ebeling et al. 2014). The main proposed mechanisms underlying increased productivity with increased plant diversity are selection effects (the chance to include highly productive species) and complementarity (facilitation between species or niche differences leading to more efficient use of space and resources). Presence of legume species for example, has a strong complementarity effect because they obtain nitrogen from the atmosphere whereas nonlegumes depend on soil mineral nitrogen (Loreau \& Hector 2001; Hooper \& Dukes 2004). Improved biodiversity experiments were designed such that complementarity and sampling effects could be disentangled (Loreau \& Hector 2001; Roscher et al. 2004). This can be achieved by incorporating a plant functional group (legumes, grasses, forbs) approach such that plant functional groups are more equally represented at each level of species richness (Roscher et al. 2004). Apart from the role of resource use complementarity by plants of different functional types a key finding of the more recent biodiversity studies is that soil biota are an important factor in the plant diversity - productivity relationship (Maron et al. 2011; Schnitzer et al. 2011). 


\section{Belowground interactions}

Microbes and nematodes are the most abundant and diverse organisms in fertile soils (Bardgett \& van der Putten 2014). Archaea, bacteria, fungi, and protists recycle organic residues back into plant available nutrients. Furthermore, microbes and nematodes occupying higher trophic positions in the soil food web can also alter the mineralisation rate by selective feeding on microbes (Hättenschwiler et al. 2005). Belowground interactions between plants and soil biota are crucial for ecosystem functioning (Bardgett \& van der Putten 2014), and loss of microbial and nematode richness can have negative impacts on functioning and stability (Griffiths et al. 2000; Wagg et al. 2014). Microbial richness is influenced by abiotic factors like soil pH (Fierer \& Jackson 2006; Prober et al. 2015) and soil type (Marschner et al. 2001; Ulrich \& Becker 2006; Ramirez et al. 2014). Moreover, multiple studies have shown that the richness and abundance of nematodes is influenced by plant species identity (De Deyn et al. 2004; Quist et al. 2016) and plant diversity (Eisenhauer et al. 2011; Cortois et al. 2017).

Soil biodiversity is typically heterogeneously distributed in space even at local scale due to the impact of soil abiotic and biotic factors such as plant effects (Ettema \& Wardle 2002; Vos et al. 2013; O'Brien et al. 2016). Especially for microbial communities, it is important to notice whether plant effects are found in bulk soil, the tiny soil layer just around the roots (rhizosphere), on plant roots (the rhizoplane), or insider the roots (the endorhizosphere) (Philippot, Raaijmakers et al. 2013; van der Heijden \& Schlaeppi 2015), as plant effects can be different between these compartments (Kowalchuk et al. 2002). Nevertheless, plant identity effects on microbial community richness and composition have been found in bulk soil (Osanai et al. 2013; Burns et al. 2015), rhizosphere soil (Kowalchuk et al. 2002; Garbeva et al. 2008) and for root-associated microbes (Becklin et al. 2012).

Plant and soil communities are engaged in dynamic interactions that can result in positive, neutral and negative feedback effects. This ongoing process is called plant-soil feedback (Bever et al. 1997a) and includes biotic and abiotic changes induced by plants and soil organisms (Bartelt-Ryser et al. 2005; Ehrenfeld et al. 2005). During growth, plants accumulate a specific soil biotic community. This conditioned soil is left as a legacy to the next plant of the same (direct plant-soil feedback) or different species (indirect plant-soil feedback). The soil biota can influence the soil and the succeeding plants in different ways, 


\section{Chapter 1}

for instance via the action of pathogens, mutualists or soil nutrient levels. The net effect of these (a)biotic interactions determines whether the effect on plant performance is negative, neutral, or positive (Van der Putten et al. 2013). Pot experiments with single plant species have demonstrated that plant-soil feedbacks are plant species-specific (Bezemer et al. 2006; Cortois et al. 2016) and plant age-specific (Kardol et al. 2013; Dudenhöffer et al. 2018). However, in natural systems, multiple plant species grow together, thereby leading to complex plant-soil feedback interactions (Kulmatiski et al. 2008; Jing et al. 2015; Lekberg et al. 2018), which may impact the dynamics of plant community composition (Bever et al. 1997; Van der Putten et al. 2013). Increasing plant species richness can positively influence plant productivity by increasing mutualistic interactions of mycorrhizal fungi (Van der Heijden et al. 1998; Wagg et al. 2011) and by reducing pathogenic soil biota (Maron et al. 2011; Schnitzer et al. 2011; Hendriks et al. 2013). However, the impacts and consequences of declining plant species richness on soil biota are still not well understood (Spehn et al. 2000; Bardgett 2002; Hunt \& Wall 2002; Bardgett \& van der Putten 2014). Because the strength and direction of the feedback can change with successional time (Kardol et al. 2006), especially long term biodiversity experiments are crucial in order to more fully understand plant-soil biota diversity effects.

\section{Species diversity and trait relations of plants and soil microbes}

In plant-soil biota interaction studies, it is often observed that plant species identity effects are stronger than species richness effects, and it is suggested that plant traits play a key role in ultimately determining ecosystem properties (Díaz \& Cabido 2001; Wardle et al. 2003). Also in plant-soil feedback studies, plant traits can be used to predict plant species responses (Baxendale et al. 2014). In addition, several plant-soil feedback studies have shown that the association between plant traits and specific microbial taxa plays an important role in determining the direction (positive, neutral, negative) and strength of the feedback (Kardol et al. 2015; Bergmann et al. 2016; Cortois et al. 2016) For example, the interaction between litter decomposability and plant-soil feedback becomes increasingly positive when mycorrhizal fungi are present, but this is not the case in the presence of pathogens (Ke et al. 2015). Other studies have reported a negative correlation between specific root length (root length per unit dry weight) and mycorrhizal colonisation (Bergmann et al. 2016; Cortois et al. 2016), or have shown that plants with thin roots (high specific root length) and poor mycorrhizal colonisation suffer most from negative plant-soil feedback (Cortois et al. 2016). However, various studies show contrasting results on the predictability of soil microbial communities by plant traits (Legay et al. 2014; Leff et al. 
2018). Because trait-based studies are relatively new and different types of plant traits (morphological, chemical, developmental, above or belowground) are used among the various studies, much remains to be learned about the predictability of soil biotic diversity and community composition by plant traits.

Loss of diversity in soil biota reduces plant diversity (Wagg et al. 2014), but how declining plant diversity affects soil biota might depend on the identity and function of the soil biota (Sugiyama et al. 2008; Millard \& Singh 2010). Morphological analysis and molecular tools have become instrumental for identifying microbes and nematodes, and grouping them to potential functions. The majority of soil bacteria and fungi are saprophytes, which recycle dead organic matter into mineral nutrients (Hättenschwiler et al. 2005). By regulating the amount and rate of mineralisation, saprophytes indirectly influence plant performance (Wardle 2004). In return, plant species richness has a positive influence on saprophyte abundance in experimental grasslands (Chung et al. 2007). Also plant traits are known to affect soil bacteria. For instance, plants with high nitrogen content have a positive effect on bacterial abundance (Orwin et al. 2010; de Vries et al. 2012) and alter bacterial community composition (Moreau et al. 2015; Sayer et al. 2017).

Multiple taxa of bacteria and fungi have been identified as symbiotic mutualists of plants. These organisms and their host plants mutually benefit from symbiosis during at least part of their life cycles. For example, nitrogen-fixing bacteria, such as rhizobia, live in root nodules of legumes. Rhizobia fix atmospheric nitrogen and transform this to plant-available ammonium, while the plant exchanges carbon in return. Soil inoculated with rhizobia increases legume biomass, which subsequently can alter plant community composition (Van der Heijden et al. 2006). Legumes often harbour multiple rhizobial species at the same time (De Meyer et al. 2011), but it is still not known whether plant species richness affects rhizobial richness in soil or in legume nodules. The most well-known fungal symbiotic mutualists with plants are mycorrhizal fungi. The most prevailing mycorrhizae in grasslands involve plant colonization by arbuscular mycorrhizal fungi (AMF). This fungal group develops hyphae into plant roots, where it colonises plant cells in which it exchanges phosphate for plant-derived sugars (Smith \& Read 2008a). Mycorrhizal colonisation influences plant performance and plant-plant competition, and can thereby change plant community composition (Van der Heijden et al. 1998). Plant identity and species richness can alter AMF abundance and richness. Although most AMF interactions are positive, there are also studies in which a neutral or negative impacts on plant performance have been observed (Klironomos 2003). Beside plant identity and species richness, also plant 


\section{Chapter 1}

functional group identity (König et al. 2010) and plant traits (Bergmann et al. 2016; Cortois et al. 2016) affect the richness and abundance of AMF.

In addition to AMF, also other fungi (Clay 1999; Aguilar-Trigueros \& Rillig 2016; Geisen et al. 2017) and bacteria (Compant et al. 2005; Schulz \& Boyle 2005; Bulgarelli et al. 2013) can live inside plant roots; these microbes are referred to as root endophytes (Schulz \& Boyle 2005). Endophytes are classified as organisms that do not cause diseases like pathogenic organisms, but rather have mutualistic or neutral interactions with their host. However, it has been argued that neutral interactions between endophytes and their host might not exist, and it is proposed that there is always a degree of antagonism (Schulz \& Boyle 2005). Most plant roots host multiple endophyte species at the same time (Vandenkoornhuyse, Baldauf et al. 2002), and plant identity and plant functional groups host specific endophyte communities (Weishampel \& Bedford 2006; Mandyam et al. 2012). However, it still remains unknown whether root endophyte richness and community composition are influenced by plant species richness.

Plant pathogens or antagonists are present among fungi, bacteria, protist and nematodes (here they are named plant-feeding nematodes), but there have not yet been plant pathogenic archaea identified (Cavicchioli et al. 2003). Such antagonists feed on living plants and are widely known from studies on agricultural crops (e.g. potato cyst nematode, phytophthora, Xanthomonas species) and natural grasslands (Chakraborty 2001). Pathogen outbreaks in natural grasslands are often less well visible than in agriculture because most grasslands consist of plant mixtures, which reduce pathogen spread (Dignam et al. 2016). Moreover, in natural grasslands, antagonist pressure is expected to be an important driver of plant community diversity (Bakker et al. 2013). It has been proposed, but rarely tested, that species rich plant communities have more antagonistic species, but in low densities (Kulmatiski et al. 2012; Bever et al. 2015; Mommer et al. 2018).

In soil, a number of nematode species and other soil (micro)fauna do not feed on live or dead plant material or residues; these therefore occupy higher trophic positions in the soil food web. Protists for example, prey on bacteria, fungi, other protists, as well as on nematodes (Geisen 2016a). Protists that are non-plant pathogenic may positively influence plant growth by selectively feeding on other soil organisms, thereby releasing mineral nutrients from the soil food web (Bonkowski 2004; Geisen 2016). Plant species identity affects protist community composition (Turner et al. 2013), and plant functional group diversity may increase protist abundance (Ledeganck et al. 2003). However, it remains to 
be determined how plant species richness affects protist species richness and community composition. All nematodes except plant feeders, occupy higher trophic positions in the soil food web. Nematodes are classified by their feeding preference: bacterivores, fungal feeders, omnivores and predatory nematodes (Yeates et al. 1993). These higher trophic groups of nematodes influence plant performance by enhancing nutrient cycling. Plant species identity, species richness and functional group richness all can influence community composition and richness of nematodes at higher trophic positions in the soil food web (De Deyn et al. 2004; Eisenhauer et al. 2011; Cortois et al. 2017).

\section{Detection and characterisation of soil biota}

High-throughput molecular tools are rapidly being developed to facilitate to the analysis of soil microbial community composition. Although previously developed molecular fingerprinting tools proved highly useful for the comparison of microbial community structure across samples, high-throughput sequencing methods have more recently become the norm. Such approaches allow for microbial communities to be interrogated in a rapid and cost-effective manner, with a much greater depth of covered, and databases with taxonomic information continue to grow and become more accessible. Even though these modern tools offer great opportunities to map the enormous number of microbial taxa in soil, a large part of the soil microbial community still remains unidentified. In addition, tools continue to be developed that can link functions to microbial taxa, based on their identity (Arndt et al. 2012; Nguyen et al. 2016) or the presence of particular genes (Enault et al. 2005; Hunter et al. 2014; Priebe et al. 2015). Such tools help to identify functional changes in soil communities; for example, to determine the degree of microbial functional overlap (Louca et al. 2018).

\section{Aims and chapters}

The overall aim of this thesis is to investigate the impact of plant community composition on richness and abundance of soil microbes and nematodes. The following research questions are addressed: (a) what is the impact of plant community changes on soil communities compared to abiotic factors, (b) what aspects of plant species and community diversity affect microbial communities, (c) which microbial groups are most sensitive to plant community compositional changes, and (d) how do changes in soil microbial communities affect plant performance and community composition? 


\section{Chapter 1}

In Chapter 2, I test how species richness and community composition of soil bacteria, fungi, archaea and protists may be related to plant species richness and to plant functional groups. It is generally assumed that plant and belowground diversity are positively related (Scherber et al. 2010), however, experiments to date have mainly focussed on plant species richness effects on particular microbial groups, for example, bacteria, fungi or AMF. Soil microbial community composition also responds to abiotic environmental factors, which complicates the search for general patterns of plant species richness on soil microbial richness and community composition among different experiments. In this chapter, also soil abiotic factors are included to help identify how strong plant induced effects on soil microbial communities are compared to soil abiotic effects.

In Chapter 3, I study root-associated fungal community composition and analyse how these communities are affected by plant trait diversity and specific plant traits. Rootassociated fungi can live on the root surface or inside plant roots (van der Heijden \& Schlaeppi 2015; Sasse et al. 2018), and are a subset of the fungal community that is present in the bulk soil (Kristin \& Miranda 2013; Goldmann et al. 2016). Plants also influence the composition of these fungal communities (Saks et al. 2014; Goldmann et al. 2016). However, there have been multiple studies on root-associated fungi in grasslands, predominantly focussing on mycorrhizal colonisation. Besides effects of plant community composition, also rooting depth will be considered in order to account for the vertical gradient of soil fertility (Fierer et al. 2003; Delgado-Baquerizo et al. 2017) and decreasing fungal richness in bulk soil (Fierer et al. 2003; Sanaullah et al. 2016). In this chapter, I will study how these factors might affect root-associated fungi assembly.

In Chapter 4, I examine how plant traits alter the richness and community composition of bacteria, fungi and nematodes from the green (living plant-based) and brown (dead plantbased) pathways in the soil food web. These pathways reflect the energy channels in which organic matter flows from the primary producer (the plant) to consumer groups (Krumins et al. 2013; Bradford 2016). Furthermore, I analyse whether plant traits and diversity effects differ between lower and higher trophic positions in the soil food web. Organisms from the green pathway interact more directly with plants, whereas organisms from the brown pathway may also feed on detritus that has already been pre-treated by other soil biota (Moore et al. 2004; Krumins et al. 2013). In addition, plant species richness effects diminished with trophic position in the food web (Scherber et al. 2010). Therefore, soil biota from the green and brown pathways and higher trophic positions may respond differently to changes in plant community composition. 
In Chapter 5, I report on a field experiment in which I test the effect of mycorrhizal networks on seedling performance in restored grasslands. Arbuscular mycorrhizal fungi (AMF) form hyphal networks that may connect multiple plants of the same (conspecific), as well as different (heterospecific) species (Francis \& Read 1995; Van der Heijden \& Horton 2009; Simard et al. 2012). Nutrients (Meding \& Zasoski 2008; Mikkelsen et al. 2008) and signalling compounds (Achatz \& Rillig 2014; Johnson \& Gilbert 2015) can then be transferred via these networks. Although experiments with individual plants generally show that AMF colonisation is beneficial for plant establishment and growth, experiments with seedlings in plant communities demonstrate that the presence of AMF does not necessarily improve seedling performance (Bennett et al. 2017) (Moora \& Zobel 1996). Mycorrhizal network effects on seedling performance have been rarely tested in field experiments. Because AMF are thought to contribute to positive plant-soil feedbacks, I additionally tested whether seedlings with positive plant-soil feedback are differentially affected by mycorrhizal networks compared to seedlings with negative plant-soil feedback.

\section{$2 \mathrm{PSR}+\mathrm{FG}$}

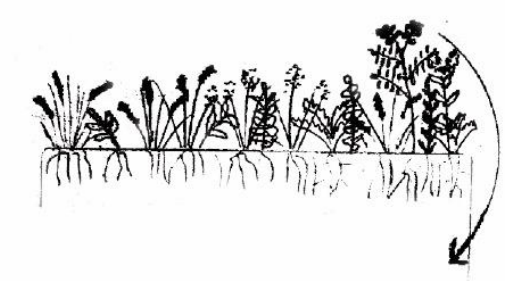

4 Traits + FD + FG + PSR

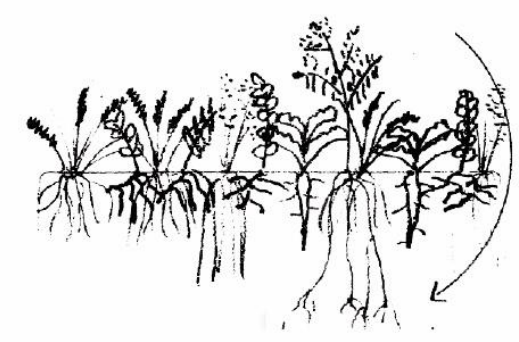

\section{Traits + FD + FG + PSR}

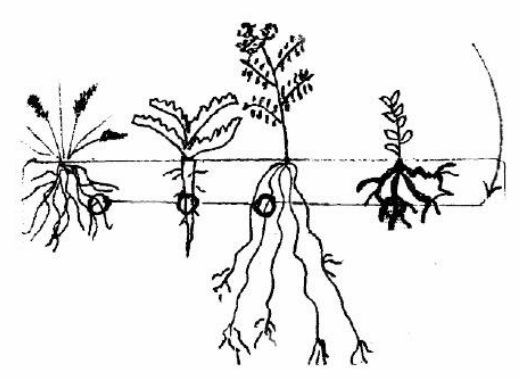

5 FG + PSF

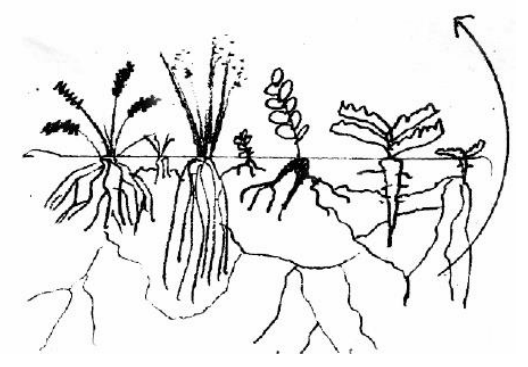

Figure 1 The research presented in the different chapters of this thesis. Numbers coincide with the given chapters. $\mathrm{PSR}=$ plant species richness, $\mathrm{FG}=$ functional groups, Traits $=$ plant specific traits, FD = functional diversity, $\mathrm{PSF}$ $=$ Plant-soil feedback. 


\section{Approach}

\section{The Jena Experiments}

The first three research chapters are based on soil and root samples taken from The Jena Experiments. The first experiment is the long-term biodiversity experiment in Jena, Germany, and one of the longer running examples of such experiments to date. The experiment was set up in 2002 to investigate the role of plant biodiversity in element cycling and in trophic interactions (Roscher et al. 2004). From a pool of 60 common European grassland species, 20x20 meter plots were established in which plant communities varying from 1 to 60 plant species were sown and maintained within a replicated experimental design of 90 plots in total. The experimental design enables to examine plant functional group and functional group diversity effects in a statistically sound approach. In 2010, an additional experiment was set up on the same field site in which, beside plant species richness, also trait diversity was manipulated experimentally, although legume species were excluded (Ebeling et al. 2014). In this Trait-Based Experiment the established plant communities have different levels of trait complementarity, with functional diversity ranging from plant communities with similar plant traits to plant communities with high trait complementarity. The plots are $3 \times 3$ meter and in total 138 plots were established. The design allows for testing the importance of selection effects (the change that a highly productive species is present in the community) and complementarity effects (increased plant performance in plant mixtures compared to what is expected from individual plants) on ecosystem processes. An advantage of the Jena Experiments is the huge amount of data collection and sharing among members, and an increasing number of datasets that have become publically available. This allows members of the research community to explore ecosystem processes in more detail without the need to perform comprehensive samplings and analyses.

\section{Veluwe restoration sites}

The final research chapter is based on experiments conducted on former agricultural fields at the Veluwe (The Netherlands), representing a gradient in stage of secondary succession. The most recently abandoned field in the succession gradient was withdrawn from 
agricultural practices 11 years ago, whereas the oldest field succession in my succession series was abandoned 28 years ago. The fields are grazed by natural herbivores (hares, rabbits, and deer) and introduced livestock (cows, horses and sheep) at low stocking density. This chronosequence has been used for a range of studies examining plant-soil interactions, for example to study how succession relates to plant-soil feedback (Kardol et al. 2006; van de Voorde et al. 2011), soil food web dynamics (Morriën et al. 2017), and fungal functional community composition (Hannula et al. 2017). These studies have shown that soil community composition and functioning change with increasing time since abandonment. These fields therefore represent a suitable location to study experimentally how mycorrhizal network affects seedlings, and how the success may depend on shifts in fungal community composition and functions. 


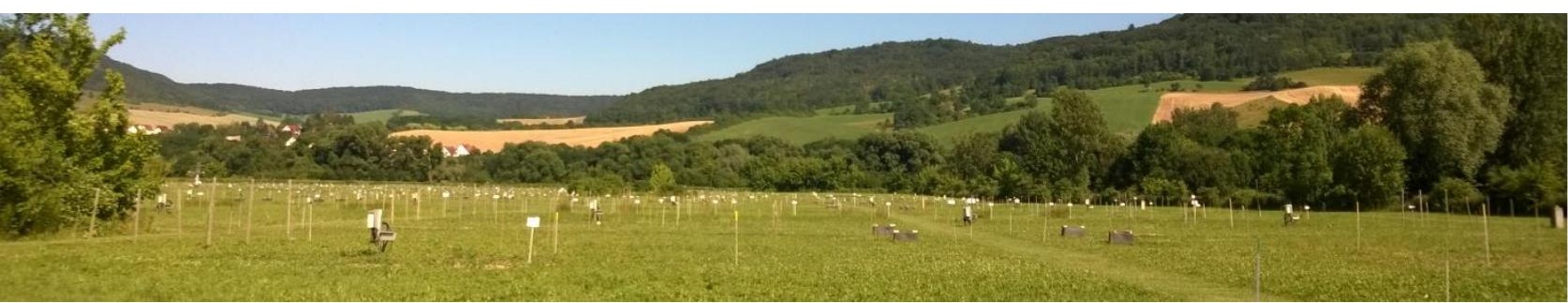

The Jena Main Experiment at high summer. 
Chapter 2

\title{
Differential responses of soil bacteria, fungi, archaea and protists to plant species richness and plant functional group
}

\author{
identity \\ Sigrid Dassen \\ Roeland Cortois \\ Henk Martens \\ Mattias de Hollander \\ George A. Kowalchuk \\ Wim H. van der Putten \\ Gerlinde B. De Deyn
}

Published in Molecular Ecology (2017), 26:4085-98. 


\section{Chapter 2}

\section{Summary}

Plants are known to influence belowground microbial community structure along their roots, but the impacts of plant species richness and plant functional group (FG) identity on microbial communities in the bulk soil are still not well understood. Here, we used 454pyrosequencing to analyse the soil microbial community composition in a long-term biodiversity experiment at Jena, Germany. We examined responses of bacteria, fungi, archaea, and protists to plant species richness (communities varying from 1-60 sown species) and plant FG identity (grasses, legumes, small herbs, tall herbs) in bulk soil. We hypothesised that plant species richness and FG identity would alter microbial community composition and have a positive impact on microbial species richness. Plant species richness had a marginal positive effect on the richness of fungi, but we observed no such effect on bacteria, archaea, and protists. Plant species richness also did not have a large impact on microbial community composition. Rather, abiotic soil properties partially explained the community composition of bacteria, fungi, arbuscular mycorrhizal fungi (AMF), archaea, and protists. Plant FG richness did not impact microbial community composition, however plant FG identity was more effective. Bacterial richness was highest in legume plots and lowest in small herb plots, and AMF and archaeal community composition in legume plant communities was distinct from that in communities composed of other plant FGs. We conclude that soil microbial community composition in bulk soil is influenced more by changes in plant FG composition and abiotic soil properties, than by changes in plant species richness per se. 


\section{Introduction}

Soil microbes are known to be critical to terrestrial ecosystem functioning and are key determinants of plant community composition and diversity (van der Heijden et al. 2008; Bardgett \& van der Putten 2014). The majority of plant-soil microbe studies have focussed on the interface between plant roots and soil, the rhizosphere (Buée, Reich et al. 2009; Philippot, Raaijmakers et al. 2013; Mellado-Vázquez et al. 2016). However, less is known about the contribution of plant communities in driving the composition and diversity of microbes in bulk soil (Vos et al. 2013). Here, we examine how plant diversity and soil abiotic properties influence bulk soil microbial community richness and composition.

Increasing plant species richness has generally been shown to lead to increased soil microbial biomass (Zak et al. 2003; Eisenhauer et al. 2010; Scherber et al. 2010) and activity (Eisenhauer et al. 2010). One explanation is that increased plant species richness diversifies the resource pool so that the biomass of a larger variety of soil microbes may be sustained (Milcu et al. 2006; Sauheitl et al. 2010). Another possibility is that increasing plant species richness enhances the amount of primary production, which in turn may increase the biomass of soil fungi and bacteria (De Deyn et al. 2011). Plant community diversity effects on ecosystems might be due to plant species richness per se or to richness of their traits, or functions (Díaz \& Cabido 2001). It has been proposed that variation in plant traits can, at least partially, explain variation in soil microbial community composition (Bardgett et al. 2014; Legay et al. 2014; Reich 2014; Cornwell et al. 2008).

Grasses, legumes, tall herbs and small herbs represent typical plant functional groups that may fulfil different roles in ecosystems because of their distinct traits (Hector 1999; Roscher et al. 2004). Several plant traits that are typical for a particular plant FG have been shown to be related to microbial community composition and functioning (Baxendale et al. 2014; Legay et al. 2014; Cantarel et al. 2015; Cortois et al. 2016). For example, specific root length (SRL) is different between small and tall herbs and SRL is related to arbuscular mycorrhizal fungi root colonisation (Cortois et al. 2016). The C:N ratio of legumes is typically lower than in other plant FGs (Abbas et al. 2013), and C:N ratio is a good predictor of litter decomposition (Chen, Mommet et al. 2017). Few studies have examined the effects of plant species richness and plant FG identity in long-term experiments in the field, and most have been limited to a subset of the soil microbial community (Waldrop et al. 2006; König et al. 2010; Schlatter et al. 2015). 


\section{Chapter 2}

When examining plant impacts on soil-borne microbial communities, it is relevant to not only examine the species richness of individual communities, i.e. $\alpha$-diversity, but also the identity and abundance of each species in the community, i.e. $\beta$-diversity, which can be expressed in (dis)similarity between microbial communities (Whittaker 1972; Anderson et al. 2011), or in community evenness (Simpson 1949). To date, few studies have attempted to relate plant species richness to microbial species richness in bulk soil, and their outcomes have been variable. While some studies have reported no correlation between species richness of plants and species richness of bacteria (Sugiyama et al. 2008; Schlatter et al. 2015) or fungi (Waldrop et al. 2006), Sugiyama (2008) found a positive correlation between plant species richness and fungal richness in bulk soil. Differences in outcomes across studies may be due to the use of different methodologies (fingerprinting, cloning and sequencing, or pyrosequencing), which can vary in depth and phylogenetic resolution (Bent et al. 2007), or differences in the soil types and plant communities examined. Potential impacts of plant diversity may also be masked by differences in other environmental variables, such as $\mathrm{pH}$, soil structure and soil moisture, which may vary across and between experimental field sites.

Although higher plant diversity may impact the heterogeneity of associated soil habitats, levels of plant and microbial species richness differ several orders of magnitude. Moreover, it is not clear if higher plant species richness should dictate higher soil microbial species richness (Vos et al. 2013). Indeed, the major observable changes in microbial communities related to differences in plant community structure and diversity originate from shifts in the relative abundances of particular microbial species, as opposed to changes in absolute microbial species richness (Waldrop et al. 2006; Schlatter et al. 2015). Soil analyses of grassland biodiversity experiments also have shown that plant functional groups can differ in their effects on the abundances of fungi and bacteria. For example, the presence of legumes generally decreased the biomass of soil fungi (Bartelt-Ryser et al. 2005; Lange et al. 2014), small herbs increased total soil microbial biomass, and tall herbs and grasses did not change microbial biomass (Strecker et al. 2015). Although such studies show that plant functional group identity can impact the abundance of soil microbes, they do not provide insight into influences on soil microbial community structure. Changes in plant community composition may have different effects on bacterial than on fungal community composition (Sugiyama et al. 2008). It has been suggested that fungal communities are linked more tightly to standing vegetation because of associations with the living plant and saprotroph 
activity (Millard \& Singh 2010), or because fungi are more responsive to soil nutrient status than bacteria (Lauber et al. 2008).

The analysis of specific microbial groups may also help to understand the impacts of plant community composition on soil microbes. Examples of interesting specific microbial groups are arbuscular mycorrhizal fungi (AMF), rhizobia, archaea and protists. Although AMF are generally thought to be rather nonspecific with respect to host range (Klironomos 2000; Jansa et al. 2008), results across gradients in plant composition would suggest at least a degree of host specificity in the field (Hedlund et al. 2003; König et al. 2010; Hiiesalu et al. 2014). Furthermore, it has been shown that also plant species identity can influence both the abundance (De Deyn et al. 2011) and identity of AMF species (Vandenkoornhuyse, Husband et al. 2002; Scheublin et al. 2004). Both findings indicate that plant community diversity and plant FG composition both can influence AMF community composition in soil.

Given the stricter host specificity of rhizobia compared to AMF, it is not surprising that the number of rhizobial species in soil has been shown to increase with increasing legume biomass (Van der Heijden et al. 2006), as legumes often harbour multiple nitrogen-fixing symbiont species at the same time (De Meyer et al. 2011). Nevertheless, the main drivers of rhizobial intraspecific and interspecific diversity have generally been shown to be abiotic soil properties such as soil nitrogen and phosphate levels (Palmer \& Young 2000), pH (Rodríguez-Echeverría et al. 2014; Van Cauwenberghe et al. 2015), and land management (Palmer \& Young 2000). Archaea have been shown to be important across various terrestrial ecosystems (Prosser 2012; Offre et al. 2013), but no studies have been reported to date that examine how their richness and community structure are impacted by plant functional group identity or diversity. In contrast, soil protist communities, which are highly species diverse and integral to soil functioning (Geisen 2016), have been reported to be responsive to plant identity (Turner et al. 2013), and to plant FG identity and richness (Ledeganck et al. 2003; Glaser et al. 2015). Clearly it may be hard to find general patterns of plant species richness and composition on soil microbial community diversity, but it is worthwhile to investigated whether or not we can find them.

The aim of the present study was to investigate how the diversity and composition of microbial communities responds to plant community diversity. These relationships were examined within the context of the large-scale plant biodiversity experiment in Jena, Germany, in which plant communities varying from 1 to 60 plant species were sown and 


\section{Chapter 2}

maintained within a replicated experimental design. We used 454-pyrosequencing of small subunit ribosomal RNA markers to determine the community structure and diversity of bacteria, archaea, fungi and protists in bulk soil samples collected eight years into this field experiment. Community patterns were examined in the light of plant species diversity, plant functional group composition and abiotic factors within the experimental field sites. We hypothesized that sown plant diversity and plant functional group diversity would have a positive effect on soil microbial richness of bacteria, rhizobia, archaea, fungi, AMF and protists. However, given the intrinsically high diversity within soil communities (Torsvik \& Øvreås 2002), we expected any such effects to be minor. In addition, we hypothesized that microbial community composition would be impacted by plant functional group, and that this effect would be stronger for fungi than bacteria.

\section{Methods}

\section{Study site, experimental design and management}

The study site used for sampling is the biodiversity long-term biodiversity grassland experiment at Jena, Germany, established in 2002. A total of 60 European grassland species (grasses, legumes, small herbs and tall herbs) were sown in plots of $20 \times 20 \mathrm{~m}$ at sowing diversity levels of $1,2,4,8,16$, and 60 plant species, and functional group richness was varied from 1 to 4 (Roscher et al. 2004). The experimental field site has 4 blocks, with block 1 situated closest to the river 'Saale' and block 4 furthest away from the river, thereby covering a soil texture gradient with different proportions of sand and silt and a $\mathrm{pH}$ gradient from 7.59 to 8.15 , with decreasing acidity with increasing distance from the river. Each block is a replication of the functional group gradient and plant species richness gradient, whereby plant identities within each plant community at each level of plant species and functional group richness vary among the blocks in order to avoid sampling effects (Roscher et al. 2004). Plots are mown twice a year and hand-weeded three times per year to remove non-sown plant species; target plant species that disappear from the plots are not re-sown. Data on plant cover was obtained by the Jena Consortium (Marquard et al. 2013). Data on total soil organic carbon (TOC) was obtained by Maike Habekost and Gerd Gleixner (doi:10.1594/PANGAEA.848944). Data for soil texture were collected by Anke Hildebrand and Jussi Baade (doi: 10.1007/s11104-014-2373-5 and doi:10.1111/j.13652486.2008.01697.x) (Steinbeiss et al. 2008; Fischer et al. 2015). An overview of the soil characteristics across the experimental site is provided (Table S1). 


\section{Soil sampling}

In September 2010, soil samples were collected across 82 experimental plots: 16 monocultures, 16 2-species mixtures, 16 4-species mixtures, 16 8-species mixtures, 14 16species mixtures, and 4 60-species mixtures. Per plot, five $2.5 \mathrm{~cm}$ diameter soil cores were taken to a depth of $15 \mathrm{~cm}$ deep at random vegetation-bearing sampling points. These five soil samples were pooled and sieved using a $2 \mathrm{~mm}$ mesh to remove plant roots and other large organic material. A subsample from each pooled sample was taken to determine soil $\mathrm{pH}$, using standard methods, and the remainder frozen and kept at $-20{ }^{\circ} \mathrm{C}$ until DNA extraction.

\section{DNA extraction and amplification}

DNA was extracted from $0.25 \mathrm{~g}$ soil with the PowerSoil ${ }^{\circledR}$ DNA Isolation Kit (MoBio, Carlsbad, CA, USA). We amplified 16S rRNA gene fragments from bacteria and archaea using the primer combination 515f/806r (Bates et al. 2011). The amplification mixture contained $0.1 \mathrm{pmol}^{-1} \mathrm{l}^{-1}$ of each primer, $1 \mu \mathrm{l}$ DNA, $200 \mu \mathrm{M}$ dNTPs, 0.056U FastStart ${ }^{\mathrm{TM}}$ High Fidelity PCR System (ROCHE), and 10x PCR buffer, in a total reaction volume of 25 $\mu 1$. The PCR amplification of $16 \mathrm{~S}$ had an initial annealing temperature of $95{ }^{\circ} \mathrm{C}(5 \mathrm{~min})$, then 30 cycles with denaturation at $95{ }^{\circ} \mathrm{C}(30 \mathrm{sec})$, annealing at $53{ }^{\circ} \mathrm{C}(1 \mathrm{~min})$, extension at $72{ }^{\circ} \mathrm{C}(1 \mathrm{~min})$, and final extension $72{ }^{\circ} \mathrm{C}(10 \mathrm{~min})$. We amplified 18S rRNA gene fragments from fungi and protists with primer FR 1 and the modified version of FF390 5'CGWTAACGAACGAGACCT-3' designed to also include the Glomeromycota (Kuramae et al. 2013). The amplification mixture contained $0.1 \mathrm{pmol} \mu \mathrm{l}^{-1}$ of each primer, $1 \mu \mathrm{l}$ DNA, $200 \mu \mathrm{M}$ dNTPs, 0.056U FastStart ${ }^{\mathrm{TM}}$ High Fidelity PCR System (ROCHE), 10x PCR buffer, $2.5 \mu \mathrm{M} \mathrm{MgCl}_{2}$, and $0.5 \mathrm{mg} \mathrm{ml}^{-1}$ bovine serum albumin. The thermalcycling program consisted of an initial annealing temperature at $95{ }^{\circ} \mathrm{C}(5 \mathrm{~min})$, followed by 30 cycles with denaturation at $95{ }^{\circ} \mathrm{C}(30 \mathrm{sec})$, annealing at $58{ }^{\circ} \mathrm{C}(30 \mathrm{sec})$, extension at $72{ }^{\circ} \mathrm{C}(1 \mathrm{~min})$, and final extention at $72{ }^{\circ} \mathrm{C}(10 \mathrm{~min})$. The primers included unique tags for the 82 plots from which the samples were taken. Amplicons were purified prior to sequencing with QIAquick PCR Purification Kit (Qiagen, Hilden, Germany) protocol, according to the manufacturer's 


\section{Chapter 2}

protocol. After quantification of PCR products, equal amounts of each sample were combined on a 96 well sequence plate, one full plate for each set of primers. The samples were subjected to Roche 454 automated sequencer and GS FLX system using titanium chemistry (Macrogen Seoul, Korea).

\section{Sequence processing}

Sequences were analysed using a Snakemake workflow (Köster \& Rahmann 2012) that follows the SOP for 454 data in Mother version 1.33.2 (Schloss et al. 2009). Flowgrams were denoised and quality filtered by using the shhh.flows command (Quince et al. 2011), which includes de-multiplexing and trimming of the flowgrams. To further reduce sequence errors the pre.cluster command was used to merge sequences that were within 2 mismatches of each other. Chimeric sequences were removed using chimera.uchime command (Edgar et al. 2011). Clustering of reads into OTUs was performed at a $97 \%$ identity threshold using the dist.seqs command and average neighbour clustering. A 97\% threshold does not allow clear species or genus distinctions of the reads, however it does provide robust diversity comparisons that avoid artificial inflation of diversity due to methodological issues such as PCR errors (Wintzingerode et al. 1997; Behnke et al. 2011). Sequences were aligned and classified with SINA (Pruesse et al. 2012) against the SILVA 119 database (Quast et al. 2013). The initial attempt to classify sequences with the RDP database (Wang et al. 2007) resulted in a large proportion of OTUs classified as bacteria but without further taxonomic depth. For each OTU a consensus taxonomy was determined based on the lowest taxonomic ancestor, using the classify.otu command. Taxonomic classification and OTU clustering data are combined into the BIOM format (McDonald et al. 2012) for further downstream statistical analysis.

\section{Data analysis}

OTUs that had two reads or fewer were removed from the dataset. Rarefaction was performed using R (version 3.0.3) (R Development Core Team) with package 'vegan' 2.2-1 (Oksanen et al. 2015), using the Rarefy command. Rarefaction curves were made to identify samples with insufficient sampling depth (Fig. S1). For bacterial and for fungal samples the cut off was set at 4000 reads per sample, resulting in 69 and 67 sequence libraries for bacteria and fungi, respectively. For archaea, the cut off was set at 750 reads per sample resulting in 77 sequence libraries, and for protists the cut off was set at 100 (66 
sequence libraries). For $\mathrm{N}$-fixers a threshold of 15 reads per sample was set (48 sequence libraries), and for AMF the cut off was at 100 reads per sample (59 sequence libraries). Prior to further analyses, the observed number of reads was rarefied to the cut off made in the previous step. Bar-plots of the relative OTU abundances of bacteria plus archaea and fungi plus protists in relation to plant species richness were made in Excel (Microsoft, 2010).

Hand weeding was necessary to maintain the plant species diversity treatments. We tested how the periodically removed plant species may have influenced the observed relationships between microbial, plant species, and plant functional group diversities. We found that the effect of actual plant species numbers (both sown and those periodically removed by hand weeding) on microbial species richness was not significant for none of the six microbial groups examined. Because removal of weed species can be seen as a soil disturbance factor, weed cover percentage per plot was included in the models described below.

To test for a correlation between plant sown diversity and OTU richness or microbial community evenness (inverse Simpson's index) a linear mixed effect model was used with block included as a random factor and $\mathrm{pH}$, TOC, soil texture, and weed cover percentage as co-variables. Sown plant diversity was fit in the models and presented in figures as a logarithm with base 2 . This was done to have a more equal distribution of step size between the levels of sown plant diversity. The model was made in $\mathrm{R}$ with the package 'nlme' (Pinheiro et al. 2015) and anova was used to produce test statistics. Corresponding figures were made in R with the package 'ggplot2' (Wickham 2009). To further investigate the positive correlation between fungal OTU richness and plant sown diversity the partitioning of OTU turnover and nestedness were calculated as described by Baselga (2010). Multiple site dissimilarity measure was used to obtain values for the partitioning of nestedness and turnover. Differences in plant sown diversity between plots was calculated with Euclidean distance. Subsequently pairwise dissimilarity measure was used to tests for a correlation between nestedness or turnover and plant sown diversity via a mantel test (1000 permutations).

To test whether plant sown diversity and abiotic soil properties influence microbial community composition, distance based redundancy analysis (db-RDA) was performed with forward selection of the explanatory plant and soil variables. Community distances were calculated with the Bray-Curtis measure and explanatory variables were included into the model if $\mathrm{P}_{\text {adj }}$ was $<0.05$. If the microbial community composition could not be 


\section{Chapter 2}

explained by any of the variables in the model, nonmetric multidimensional scaling (NMDS) was used to show the sample distribution of the microbial communities in the different plots. All plots with mixtures of plant functional groups were excluded when testing for differences in microbial diversity between the plant functional groups across the plant species richness gradient.

To test for a correlation between plant functional group richness and OTU richness a linear mixed effect model was used with block included as a random factor and $\mathrm{pH}$, TOC, soil texture, and weed cover percentage as co-variables. The model was made in $\mathrm{R}$ with the package 'nlme' (Pinheiro et al. 2015) and anova was used to produce test statistics. To test the responses of OTU richness to plant functional group identity, Poisson generalised linear model (GLM) was used with sown plant diversity and plant functional group identity and their interaction as predicting factors, while using $\mathrm{pH}, \mathrm{SOM}$, soil texture, and weed cover percentage as co-variables; block effect was not included because the functional groups were not evenly distributed over the blocks. The interaction between plant sown diversity and plant functional group was removed from the model if the reduced model was a better predictor for microbial OTU richness. Post-hoc Tukey's test was used to identify which plant functional groups were different from one another. To test for plant functional group identity effects on microbial community composition, db-RDA with forward selection was used as described above. The NMDS, db-RDA analyses and corresponding plots were performed in CANOCO (version 5.0), and the linear models and graphs were made in $\mathrm{R}$ with package 'ggplot2'(Wickham 2009).

\section{Results}

\section{Diversity of soil bacteria and fungi}

Amplification of 16S rRNA gene fragments yielded in total 4025 bacterial, 23 archaeal and 826 unclassified OTUs, respectively, at a 97\% similarity threshold. Amplification of eukaryotic 18S rRNA fragments yielded 431 fungal, 174 protist, 9 plant, and 374 unclassified OTUs, respectively. All unclassified OTUs and OTUs of plant origin were excluded from further analyses. We could classify $84 \%$ of the bacterial and $71 \%$ of the fungal OTUs to at least an order level of taxonomic resolution. For the bacteria, 39\% of OTUs could be identified to the genus level, whereas only $11 \%$ of the fungi could be identified to at least the genus level. 
The most dominant taxonomic group of bacteria was the Chloroflexi, based on the relative abundance of the sequence reads (Fig. 1a). The most diverse bacterial groups were Proteobacteria and Planctomycetes with 1045 and 655 OTUs, respectively (Fig 1a). A total of 19 putative rhizobial OTUs were recovered across the experimental fields (Table S2). The most dominant taxonomic group of eukaryotes was Ascomycota, which was also the most diverse fungal group with 177 OTUs (Fig. 1b). In total 19 AMF OTUs (phylum Glomeromycota) were recovered across all plant communities (Fig. 1b). Of the main protist supergroups, Rhizaria were well represented in our data, even though the FR1 primer used has some fungal specifity. Although protists represent a relatively small proportion $(\approx 2 \%)$ of the total eukaryotic community, their diversity was considerable, with 174 detected OTUs.

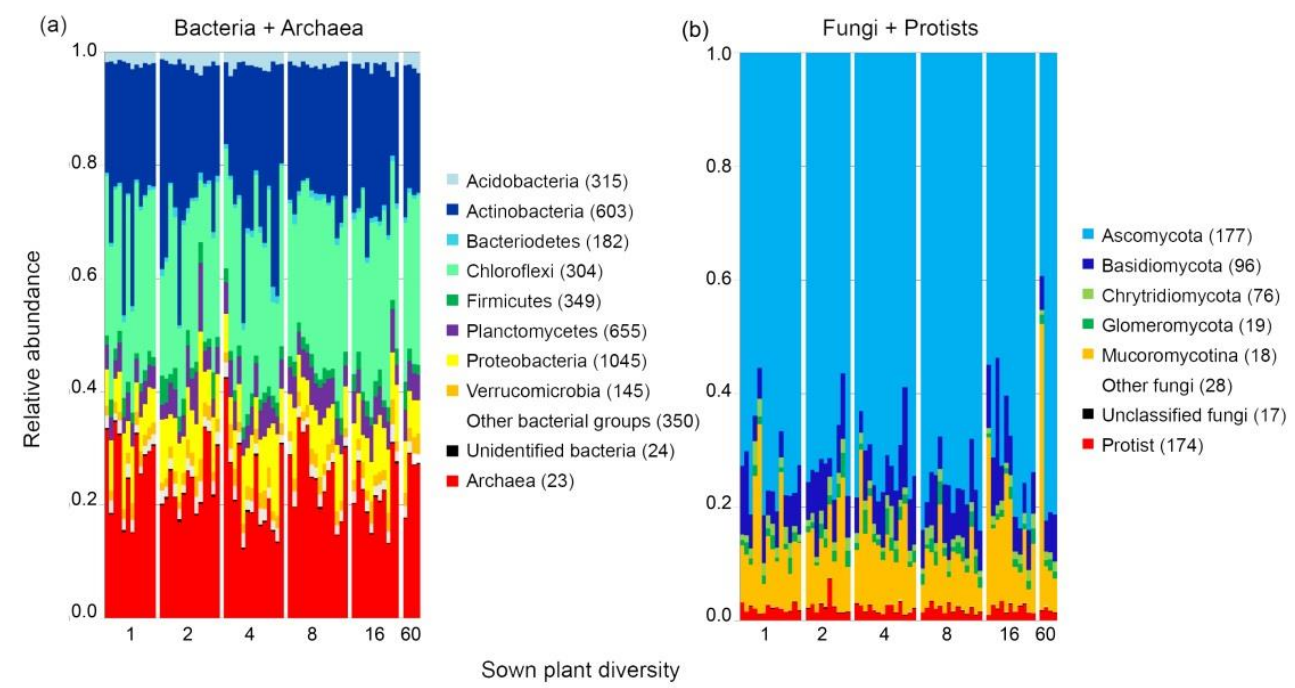

Figure 1 Relative composition of the (a) prokaryotic and (b) eukaryotic communities in relation to sown diversity. Each stacked bar represents the observed microbial taxonomic groups in a plot as a fraction of all observed taxa. Plots were grouped according to level of sown diversity $\left(1,2,4,8,16\right.$, and 60 plant species.plot $\left.{ }^{-1}\right)$ that was artificially maintained by hand-weeding. In the legend, taxonomic groups are followed by a number between brackets, which indicates the total number of different OTUs that could be classified to that group.

\section{Sown plant diversity effects on microbial richness and community composition}

The mean number of bacterial OTUs per plot did not increase significantly with increasing sown plant diversities $\left(\mathrm{F}_{1,58}=1.793, P=0.186\right)$ (Table S3, Fig. 2a). Fungi, however, showed a trend of increasing OTU richness with increasing sown plant diversity $\left(\mathrm{F}_{1,56}=\right.$ 3.960, $P=0.052$ ) (Table S3, Fig. 2d). There was also no correlation observed between sown plant richness and the OTU richness of rhizobia $\left(\mathrm{F}_{1,37}=0.517, P=0.477\right), \operatorname{AMF}\left(\mathrm{F}_{1,48}\right.$ 
$=0.081, P=0.777)$, archaea $\left(\mathrm{F}_{1,66}=0.524, P=0.472\right)$, or protists $\left(\mathrm{F}_{1,55}=0.275, P=0.602\right)$

(Table S3, Fig. 2b, e, c, f).

Neither of the abiotic soil properties measured, nor the disturbance of the soil expressed in weed cover, showed a significant correlation with microbial richness parameters (Table S3). To investigate the trend of increased fungal OTU richness and sown plant diversity further, the partitioning of nestedness and turnover was calculated. Turnover $(\mathrm{r}=0.036, P=$ $0.324)$ and nestedness ( $r=0.035, P=0.272)$ were not significantly correlated to sown plant diversity. Calculations based on multiple site dissimilarities showed that the majority of variation is partitioned to turnover $\left(\beta_{\mathrm{JTU}}=0.958\right)$ and not to nestedness $\left(\beta_{\mathrm{JNE}}=0.006\right)$.

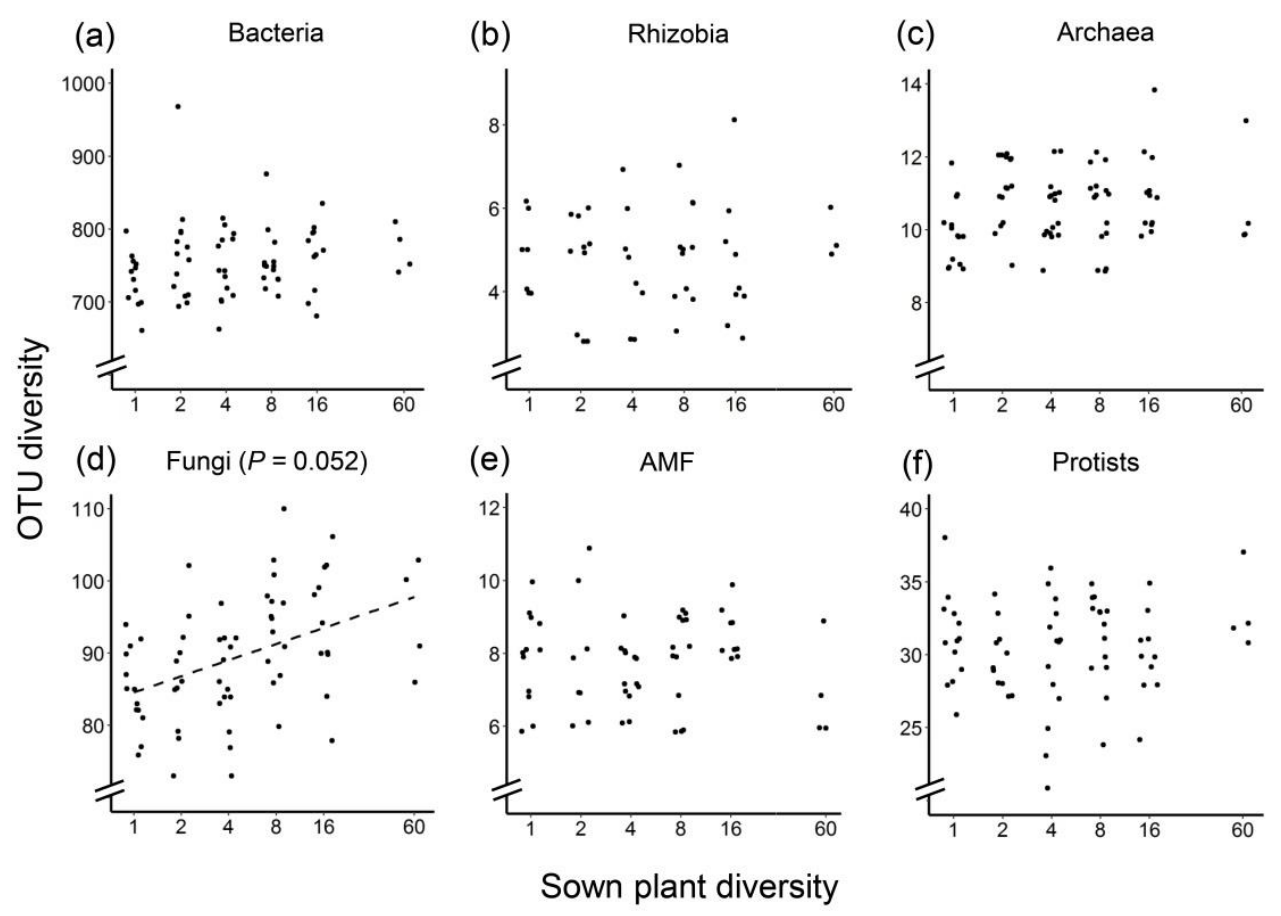

Figure 2 Relationship between (log) plant sown diversity and OTU diversity of bacteria (a), rhizobia (b), archaea (c), fungi (d), AMF (e), and protists (f). To avoid overlap, the dots in the graph are spread around their actual value. The trend line in figure $2 d$ was based on fixed effects only.

Community evenness is a measure for how well abundance is distributed over all species in the communities. Higher levels of evenness represent a community with similar abundances per species, whereas low levels of evenness represent communities with more extreme variation in abundances per species. Plant sown diversity was not correlated to bacterial $\left(\mathrm{F}_{1,58}=0.194, P=0.661\right)$ or fungal $\left(\mathrm{F}_{1.56}=1.704, P=0.197\right)$ community evenness (Table 
S4, Fig. S2a, d). Plant sown diversity was positively related with evenness for rhizobial communities $\left(\mathrm{F}_{1,37}=5.280, P=0.027\right)$ (Fig. S2b, Table S4), but there was no relation between plant sown diversity and community evenness of AMF, archaea or protists (Fig. S2; Table S4). Weed cover percentage, soil texture and TOC influenced microbial community evenness (Table S4). Bacterial evenness was unaffected by the measured soil abiotic factors, whereas fungal evenness decreased with increasing percentage weed cover. Rhizobial and AMF community evenness were also unaffected by soil abiotic factors. Community evenness of archaea was positively correlated to the percentage TOC $\left(\mathrm{F}_{1,66}=\right.$ $14.801, P=<.001)$. Soil structure affected evenness of the protist communities, with higher evenness related to a higher sand fraction $\left(\mathrm{F}_{1,55}=4.197, P=0.045\right)$ and lower community evenness with an increased fraction of silt $\left(\mathrm{F}_{1,55}=4.232, P=0.044\right)$ and clay $\left(\mathrm{F}_{1,55}=\right.$ 4.209, $P=0.045$ ).

Sown plant diversity had no effect on community composition on any of the microbial groups investigated, but abiotic soil properties did. The community composition of bacteria and fungi was influenced by soil sand fraction, which explained $4.7 \%$ of the variation in community composition of bacteria (db-RDA pseudo-F $\left.=3.3, P_{a d j}=0.012\right)$, as well as $4.7 \%$ for fungi (db-RDA pseudo-F $=3.2, P_{a d j}=0.014$ ) (Fig. 3a, d). The composition of the rhizobial communities was not related to any of the abiotic soil properties measured (Fig. $3 \mathrm{~b}$ ). Sand proportion could explain 6.75 of the variation in AMF communities (db-RDA pseudo-F $=4.1, P_{a d j}=0.012 ; \mathrm{Fig}$. 3e). Archaea communities responded to percentage sand $\left(\mathrm{db}-\mathrm{RDA}\right.$ pseudo-F $\left.=20.5, P_{a d j}=0.012\right)$ and TOC $\left(\mathrm{db}-\mathrm{RDA}\right.$ pseudo-F $\left.=7.6, P_{a d j}=0.012\right)$. Sand and TOC percentage together explained $28.7 \%$ of the variation in archaeal community composition (Fig. 3c). Protist communities were influenced by weed cover percentage (dbRDA pseudo-F $\left.=2.0, P_{a d j}=0.042\right)$ and sand (db-RDA pseudo-F $\left.=2.2, P_{a d j}=0.014\right)$. TOC and sand percentage together explained $6.3 \%$ of the variation in protist community composition (Fig. 3f).

\section{Plant functional group (FG) effects on microbial richness and community composition}

Plant FG richness did not significantly affect OTU richness of any of the microbial groups (Table S5). Abiotic soil properties also did not influence microbial richness, which is in line with results presented above (Table S3), except for weed cover percentage. Fungal OTU richness decreased with increasing weed cover percentage $\left(\mathrm{F}_{1,56}=5.259, P=0.026\right)$ (Table S5). 


\section{Chapter 2}

To test the effect of plant FG identity on microbial richness and community composition we confined analyses to plots with plant species belonging to only a single plant FG. Identity of plant FG significantly affect the OTU richness of bacteria, with significant differences between all plant FGs except between grasses and tall herbs (Table S6, Fig. S3a). Plant FG identity had no effect on OTU richness of fungi or any of the other microbial groups examined (Table S6, Fig. S3).

(a)

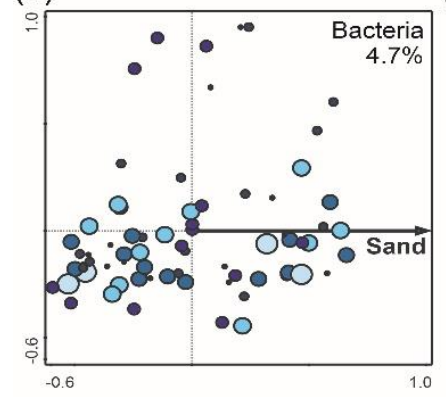

(d)

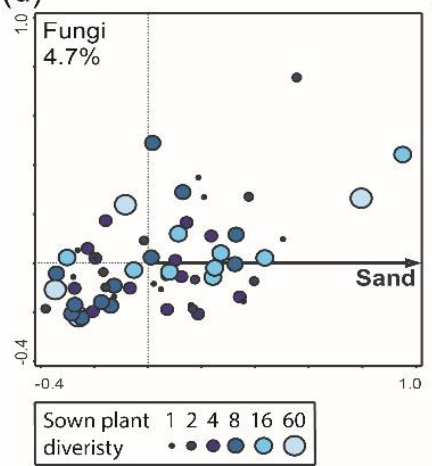

(b)

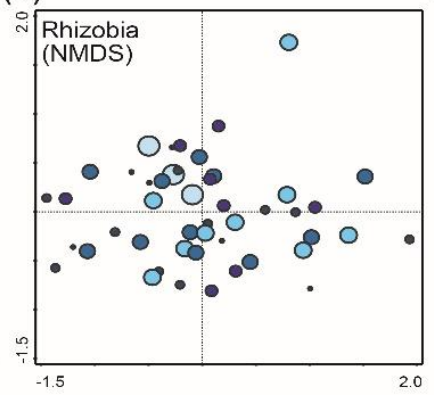

(e)

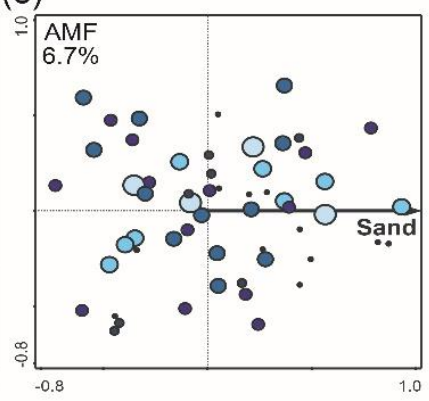

(c)

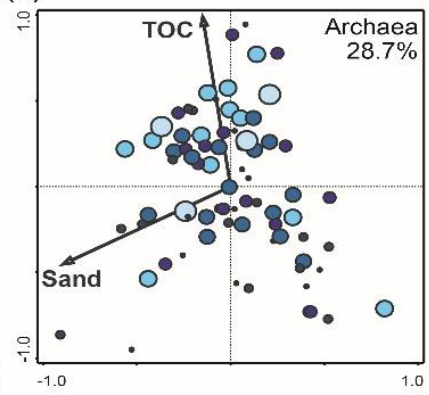

(f)

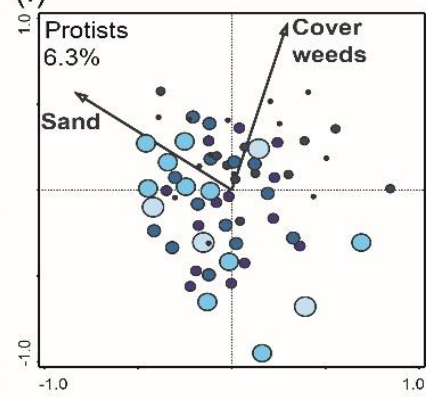

Figure 3 Distance-based redundancy analysis (db-RDA) plot showing the relationship of abiotic soil factors and (log) plant sown diversity (SD) to community composition of bacteria (a), archaea (c), fungi (d), AMF (e), and protists (f). The ordination is based on Bray-Curtis distance. With forward selection factors were chosen that significantly $\left(P_{a d j}<0.05\right)$ contributed to the model. In each window the percentage of explained variation is shown. Rhizobial community composition could not be explained by any of the factors measured, therefore a nonmetric multidimensional scaling (NMDS) of the community is showed instead (b).

Plant functional group identity did not explain a significant proportion of the variation in bacterial, fungal or rhizobial community composition (Fig. 4). In contrast, AMF communities in legume plots were however distinct from those in grass and herb plots (Fig. $4 \mathrm{e}$ ), and explained $16.1 \%$ of the variation in AMF communities (db-RDA pseudo-F $=4.2$, 
$\left.P_{a d j}=0.02\right)$. Archaeal communities in legume plots were also distinct from those in grass and herb plots (Fig. 4c), and FG explained $14.0 \%$ of the variation in archaeal communities $\left(\mathrm{db}-\mathrm{RDA}\right.$ pseudo-F $\left.=6.7, P_{a d j}=0.02\right)$. Plant FG identity had no significant explanatory power in relation to protist community composition (Fig. 4f). Abiotic soil properties did not explain a significant proportion of the variation in bacterial, rhizobial, AMF or protist community composition. For the fungi and archaea abiotic properties appeared relevant: percentage sand explained $9.0 \%$ of fungal community composition ( $\mathrm{db}-\mathrm{RDA}$ pseudo-F $=$ 2.6, $P_{a d j}=0.02$ ) (Fig. $4 \mathrm{~d}$ ), and $24.9 \%$ in archaeal community composition (db-RDA pseudo-F $=9.9, P_{a d j}=0.02$ ).

(a)

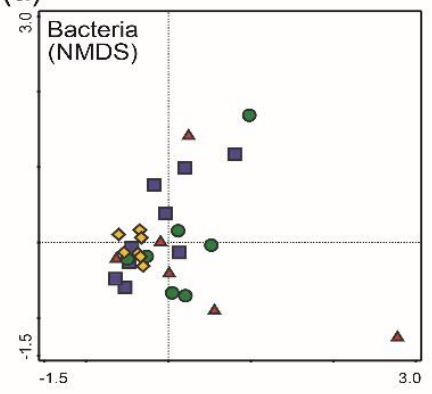

(d)

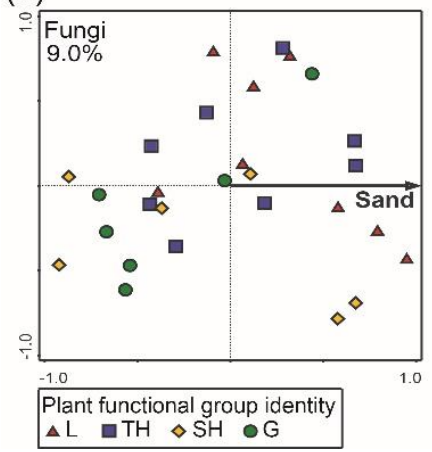

(b)

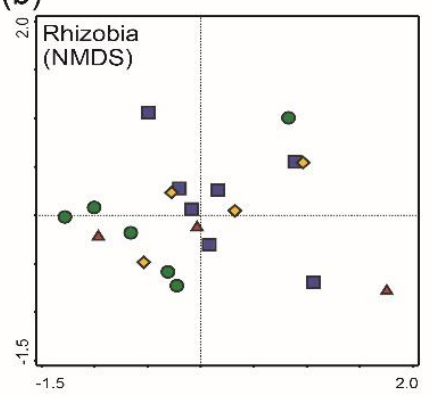

(e)

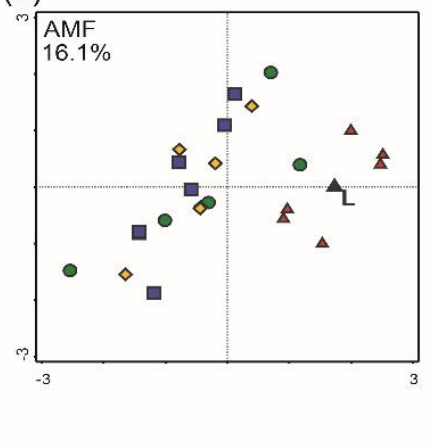

(c)

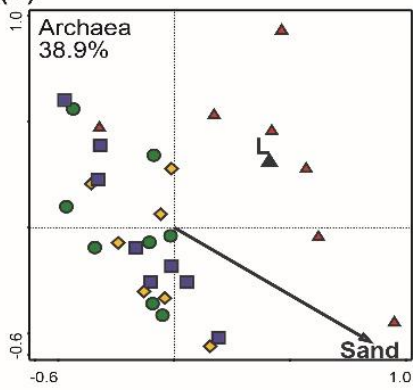

(f)

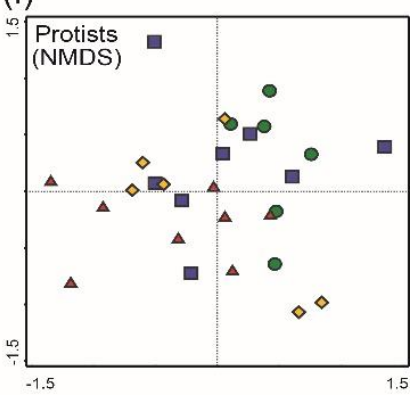

Figure 4 Distance-based redundancy analysis (db-RDA) plot showing the relationship of abiotic soil factors and plant functional group identities to community composition of archaea (c), fungi (d), and AMF (e). The plant functional groups tested were grasses (G; green), legumes (L; red), small herbs (SH; yellow) and tall herbs (TH; blue). The ordination is based on Bray-Curtis distance. With forward selection factors were chosen that significantly $\left(P_{a d j}<0.05\right)$ contributed to the model. In each window the percentage of explained variation is shown. Community composition of bacteria (a), rhizobia (b), and protists (f) could not be explained by any of the factors measured, therefore a non-metric multidimensional scaling (NMDS) of the community is showed instead.

\section{Discussion}




\section{Chapter 2}

Our study is one of the first to simultaneously examine impacts of plant species richness and plant functional group identity on richness and community composition of bacteria, fungi, archaea, and protists in a long-term controlled grassland biodiversity field experiment. Although there have been several studies analysing the effects of plant diversity on subsets of the microbial community in bulk soil (Grüter et al. 2006; LeBlanc et al. 2015; Schlatter et al. 2015), few have been able to examine plant diversity impacts on a range of microbial groups across a well-established experimental gradient of plant species diversity. Another important feature is that we targeted our sampling and analyses to bulk soil samples, as opposed to the rhizosphere, where plant-microbe interactions would be expected to be stronger. Previous studies have shown that plant diversity effects may be limited to the rhizosphere (Kowalchuk et al. 2002), although plant diversity effects have also been found in bulk soil (Sugiyama et al. 2008; Schlatter et al. 2015). Here, we observed some evidence of plant community diversity on bulk soil microbial diversities, but such effects were rather limited and not observed across all microbial groups examined.

\section{Microbial species richness}

Given the high diversity of soil-borne microbial communities, we did not expect large impacts of plant species richness per se on total soil microbial diversity. Rather, plant communities with disparate plant traits and functional groups were expected to differentially affect the relative abundance of specific microbial groups in the soil. Regarding microbial species diversity, our results showed that fungal richness responded positively to plant sown diversity. Similar results for fungi and not for bacteria were found by Sugiyama et al. (2008) in a semi-natural grassland system. The positive plant-fungi diversity interaction may be due to selective effects associated with plant-fungal interactions (including AMF) and selection of saprotrophs by specific litter traits (Millard \& Singh 2010). It may also be the case that bacteria experience more stringent top-down control than fungi making plant diversity a less important driver of bacterial diversity (Wardle 2002).

Our results are in contrast with a study on bacterial diversity in soils of the Cedar Creek biodiversity experiment, as Schlatter et al. (2014) reported a negative relationship between bacterial and plant species richness as determined by 454-pyrosequencing. The authors attributed this finding to increased resource competition in monocultures, which would favour antagonistic communities and subsequently drive higher bacterial diversity, as proposed by Kinkel et al. (2011). One important aspect that may explain the different 
results between our study and that of Schlatter et al. (2015) is the sampling design. Where Schlatter et al. (2015) collected soil cores at the base of four target species, we sampled the soil at random locations within each plot. Alternatively, the soil type, soil history and plant species used may also differentially impact the development of bacterial diversity across these systems.

Contradictory to our first hypothesis, we did not observe a positive relationship between plant FG richness and microbial species richness. There are studies that tried to relate plant FG diversity to microbial basal respiration (Strecker et al. 2015) and to microbial biomass (Bartelt-Ryser et al. 2005; Lange et al. 2014), but we are not aware of any comparative studies for microbial species richness. With increasing plant FG richness, the number of plant traits also increases. Apparently, plant trait diversity does not necessary impact microbial diversity in the bulk soil.

With respect to the impact of different plant functional groups we found that bacterial richness responded significantly to plant functional group identity. This contrasts results from a study in a steppe ecosystem, which showed that grasses and perennial forbs did not differentially affect bacterial species diversity (Zhang et al. 2015). One explanation may be the absence of legumes in their study as legumes were generally the plant functional group to which the microbial groups responded strongest. However, we also found bacterial community richness to differ between short herbs and grasses and between short herbs and tall herbs. The underlying explanation for this is yet unknown.

Our study did not reveal a significant relationship between plant diversity and the diversity of AMF, whereas a previous study by König et al. (2010) reported such a relationship within the same Jena biodiversity experiment as we examined in the current study. Such a positive relationship was also observed by Hiiesalu et al. (2014). In contrast, Lekberg et al. (2013) actually reported a negative relationship between plant species richness and AMF species richness. These opposing results may be due to a number of factors that differ with our study including the different vegetation types studied (Öpik et al. 2008), different samples sources (within roots or soil) (Saks et al. 2014) or the different methods applied to determine AMF community richness (König et al. 2010). With respect to König et al. (2010), it should also be noted that the samples examined in our study were taken three years later and changes in AMF communities may have occurred in the intervening time. Furthermore, the season of sampling (spring vs late summer) (Dumbrell et al. 2011; Bennett et al. 2013) may also have contributed to the differential results observed. 


\section{Chapter 2}

\section{Microbial community composition}

Our results showed that microbial community composition was significantly influenced by plant community composition, with stronger effects of plant functional group identity than sown plant diversity. However, this effect was not present in all microbial groups but notable in communities of rhizobia, AMF and archaea. We found that sown plant diversity was positively correlated with rhizobial community evenness, but not with evenness of the other microbial groups examined. Because rhizobia are associated with legumes, the larger number of legume species at higher sown plant diversity levels could have caused the rhizobial community to become more similar. In contrast to our results, a previous study that examined the same field experiment showed that the evenness of both bacterial and fungal communities increased with sown plant diversity (Lange et al. 2015). This contrasting result may be because of the fact that the study of Lange et al. (2015) was based on terminal restriction fragment length polymorphism (TRFLP) data, which provides a coarse level of taxonomic analysis of the community, and is only able to examine the most dominant community members.

Because of the tight association between rhizobia and legumes, we expected differently composed rhizobial communities in legume plots compared to the other plant FG plots. However, this was not the case. This may be due to the fact that rhizobia of specific species accumulate inside root hair cells (Gage 2004), and not in bulk soil. AMF and archaeal community compositions in legume plots were different from those of other plant FG plots. These findings for AMF are in line with a study on plant roots by Scheublin et al. (2004) who showed that AMF communities were different between legume and non-legume roots of plants from a nutrient-poor dune grassland system. Here, we show that, also in richer soil, and in the bulk compartment legumes accumulate distinct AMF communities. Previous work in the Jena experiment (König et al. 2010), showed that also in mixed plant communities presence of legumes, and to a lesser extend presence of small herbs, significantly affected the AMF community composition.

It is not well understood why AMF communities of legumes should differ from nonlegumes (Veresoglou et al. 2012). A possible mechanism may be related to differences in nitrogen and phosphorus stoichiometry of legumes as compared to other plants, due to the activities of nitrogen-fixing bacteria, leading to a shift from $\mathrm{N}$ - to P- limitation (Aerts \& Chapin 1999; Roscher et al. 2011). This may select for more efficient P-acquiring AMF taxa in legumes. Community composition of archaea in legume plots differed from other 
plant FGs, which could be partially explained by soil carbon or nitrogen concentrations. Presence of legumes may decrease soil carbon concentrations (Lange et al. 2015), and our data show that archaeal community composition is partially explained by TOC. Another possibility is that the increased nitrogen levels arising from legume growth (Spehn et al. 2002) led to changes in the abundance or diversity of ammonia-oxidising archaea. However, the taxonomic depth to which we could assign OTUs did not allow assessing whether affected populations were indeed ammonia oxidisers.

The absence of any effect of sown plant diversity or plant FG identity on protist community composition is remarkable considering that effects of plant identity (Turner et al. 2013) and plant FG (Ledeganck et al. 2003; Glaser et al. 2015) have been reported before. Protist community composition might not be directly linked to plant community composition but indirect via plant induced changes in abundance and composition of bacterial, fungal and nematode communities, which are all both prey and predators of protists (Geisen 2016). In addition, our protist results represent mainly the rhizarial community, whereas plant induced changes in the other protist supergroups could not be analysed.

\section{Sown plant diversity versus plant functional group}

Interestingly, the influence of plant FG identity on microbial community composition was larger than the influence of sown plant diversity. Diversification of the plant community allowed new fungal species to establish. However, these additional microbial species are probably still relatively low in abundance because their effects are not visible in ordination analyses. The fungal groups that fulfill more general ecological roles in bulk soil, such as decomposition of soil organic matter, are probably present in relatively high abundance across both the low and high plant species richness plots. However, the low abundance species cannot be considered as unimportant because rare microbes have been shown to serve important functions in soil (Hol et al. 2013; Philippot, Spor et al. 2013; DelgadoBaquerizo, Giaramida et al. 2016).

Plant FG identity had effects on microbial community composition with a notable role for legumes. Plant FGs differ in litter quality, which is correlated to litter decomposition rate (Chen, Mommet et al. 2017), and can alter the abundance of bacteria and fungi in soil differently (Bezemer et al. 2006; Chen et al. 2008; Güsewell \& Gessner 2009; Orwin et al. 2010). Beside effects of plant FG on soil nutrient status, we also expected to find plant FG phylogenetic effects on soil microbial community diversity too, although we did not test this explicitly. Since grasses are phylogenetically more related we expected their associated 


\section{Chapter 2}

microbial communities to be less variable across different grass plots compared to short or tall herb species. This expectation was not borne out from our results. Thereby our results illustrate that also species from one plant family can create diverging effects on soil microbial communities so that quantification of specific traits is warranted to understand the underlying mechanisms.

\section{Impacts of soil properties}

Soil texture explained part of the variation in community composition for all the microbial groups analysed, except rhizobia. Soil texture can influence microbial richness (Sessitsch et al. 2001), abundance (de Vries et al. 2012), and community composition (Lauber et al. 2013) via soil physical (eg pore size) and chemical properties. These factors can affect water holding capacity, drainage, the distribution of food resources, and predator access to prey (Ritz \& Young 2004). Why rhizobia remained unaffected by the measured soil properties in our experiment cannot be well explained. Rhizobial community composition is known to respond to abiotic soil factors like $\mathrm{pH}$ (Van Cauwenberghe et al. 2015). However, in the Jena experiment the $\mathrm{pH}$ gradient is relatively narrow and relatively high ( $\mathrm{pH}$ 7.6-8.2). In the majority of the earlier soil microbial community studies, $\mathrm{pH}$ has been found to be a major factor in directing microbial community composition (Dumbrell et al. 2010; Rousk et al. 2010; Prober et al. 2015; Tedersoo et al. 2015; Zhalnina et al. 2015). These studies have been conducted across a larger soil $\mathrm{pH}$ range and on average at a lower $\mathrm{pH}$ than our study. Thereby our study supports the idea that small variations in $\mathrm{pH}$ (when slightly above neutral $\mathrm{pH}$ ) do not have such a strong effect on microbial communities.

\section{Conclusions}

We found in our long-term grassland biodiversity experiment that increasing plant species richness led to higher fungal species richness in bulk soil, but had no impact on the richness of AMF, bacteria, protists, or archaea. Plant species richness also did not significantly alter the community composition of bacteria, fungi and archaea. However, we found that plant FG identity do significantly impact species richness of bacteria, as well as the community composition of AMF and archaea with a notable role for legumes. We conclude that soil microbial community composition in bulk soil can be influenced more by changes in plant FG composition and abiotic soil properties, than by changes in plant species richness per se. 
Plant-soil microbe diversity linkages 


\section{Supporting information}
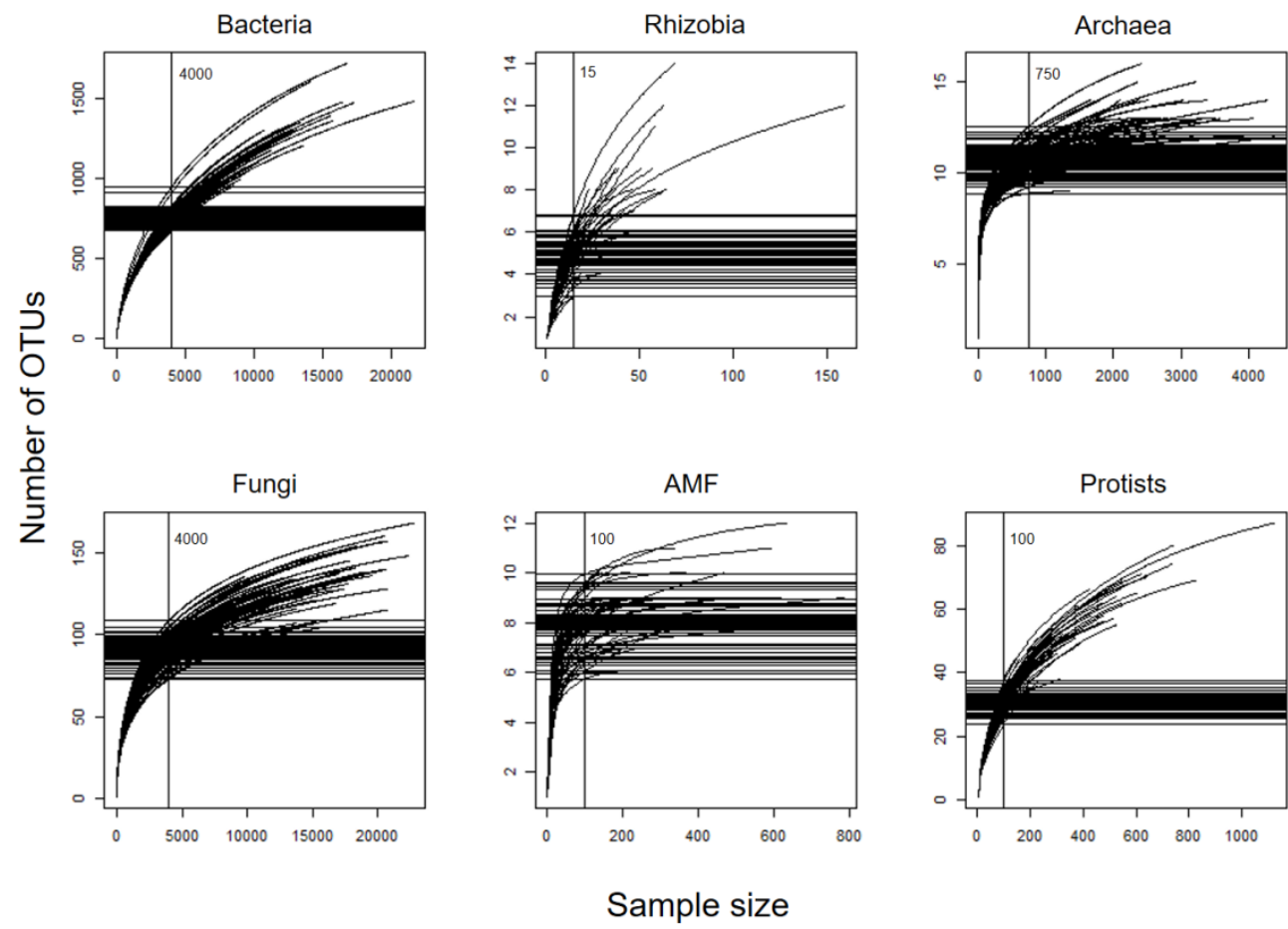

Fig. S1 Rarefaction curves for the six microbial groups (Bacteria, Rhizobia, Archaea, Fungi, AMF, Protists). Each curved line represents one sample, the vertical line and the value in the graph shows the cut-off for minimal number of reads per sample used for further analysis, the horizontal lines are the number of OTUs in each sample for the chosen cut-off. 


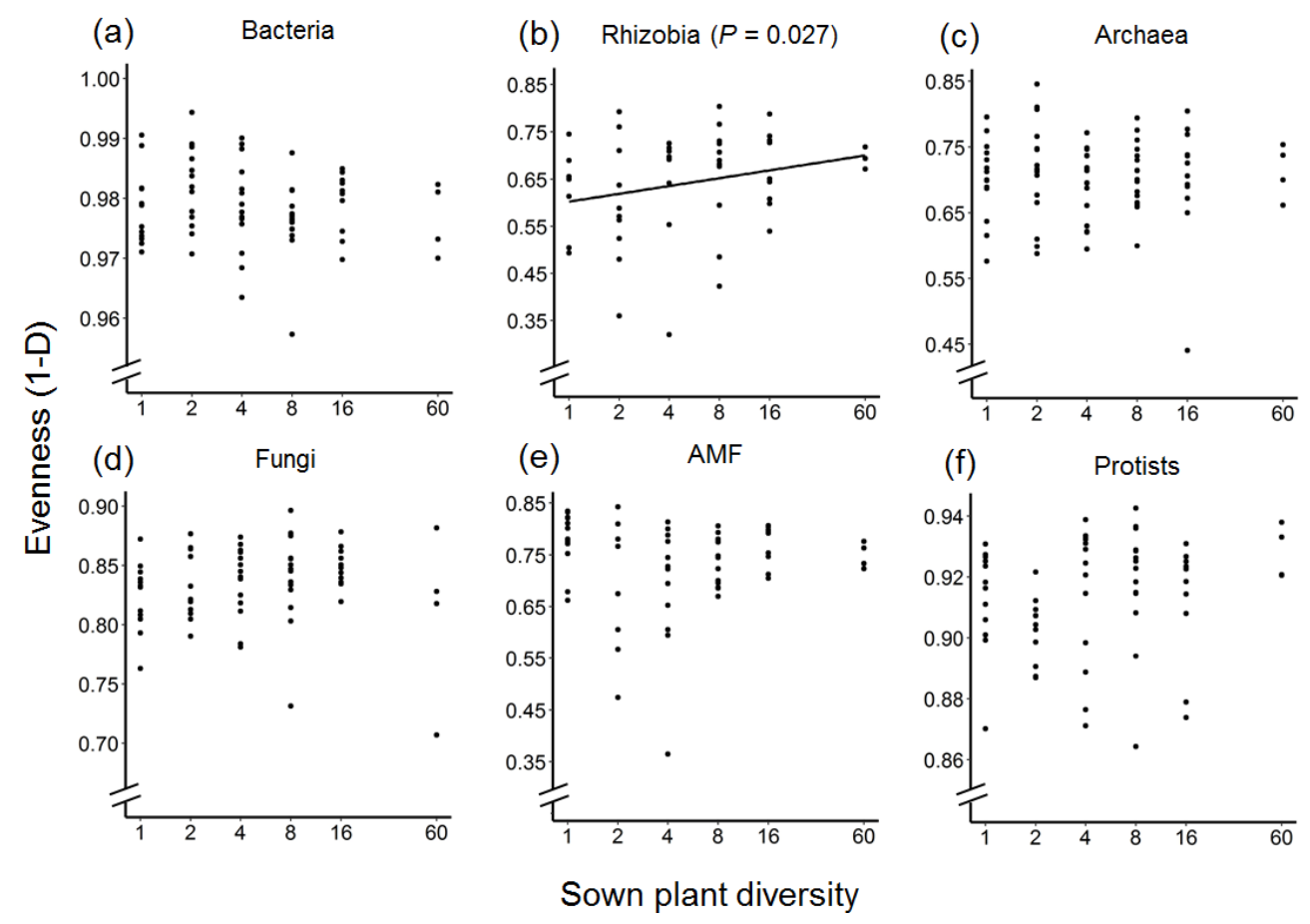

Fig. S2 Relationship between (log) plant sown diversity and community evenness (1-D) of bacteria (a), rhizobia (b), archaea (c), fungi (d), AMF (e), and protists (f). The sown plant diversity values on the x-axis are original sown plant diversity values on a log scale. 

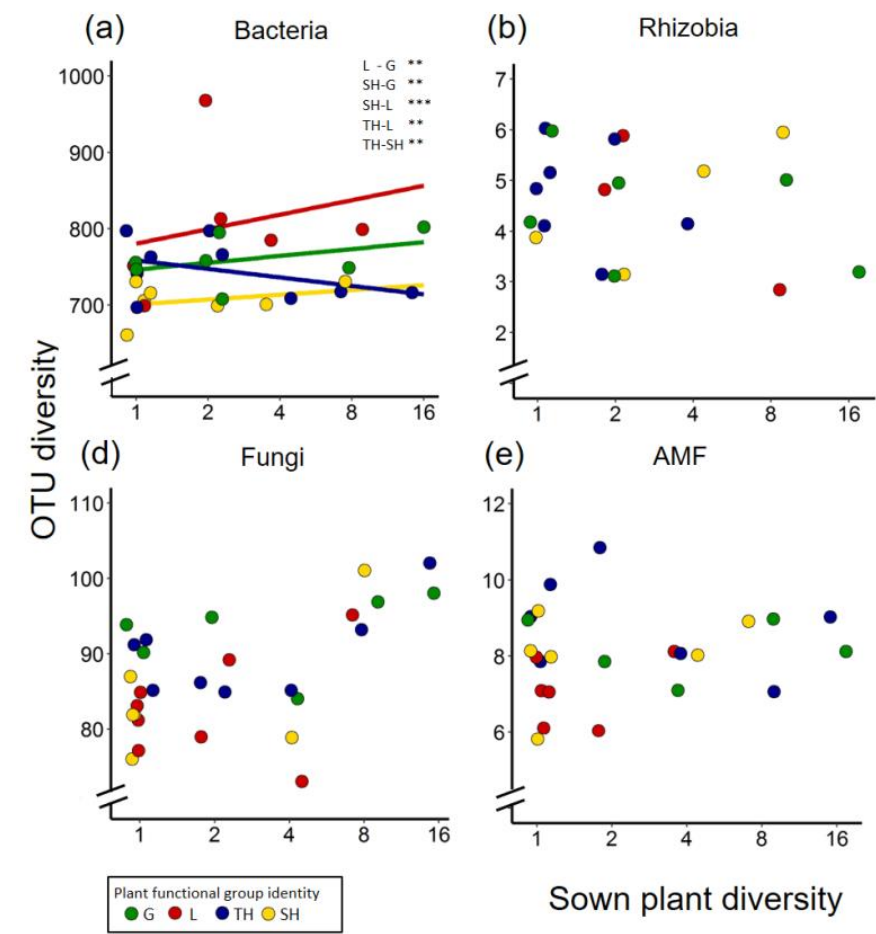

(c) Archaea

(e)
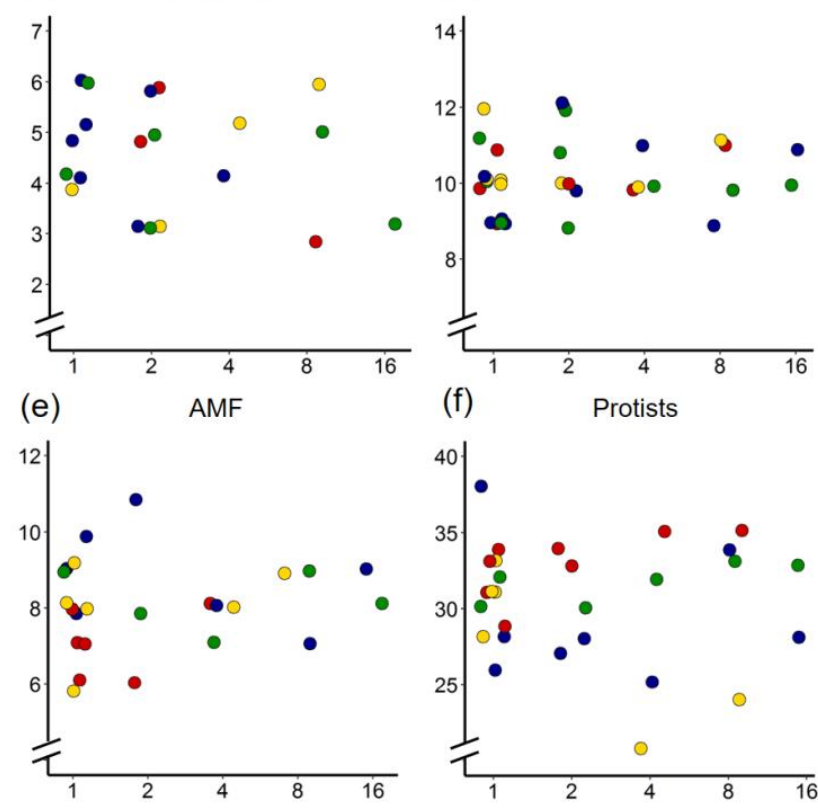

Fig. S3 Relationship between (log) plant sown diversity, plant functional group identity, and OTU diversity of bacteria (a), rhizobia (b), archaea (c), fungi (d), AMF (e), and protists (f). The plant functional groups tested were grasses (G; green), legumes (L; red), tall herbs (TH; blue) and small herbs (SH; yellow). Number of asterisks denotes significance of contribution $\left(P<0.001^{* * *}, P<0.01^{* *}\right)$. The sown plant diversity values on the $\mathrm{x}$-axis are original sown plant diversity values on a log scale. 


\section{Plant-soil microbe diversity linkages}

Table S1 Overview of the soil characteristics across the experimental field site.

\begin{tabular}{lccccc}
\hline & TOC & $\mathrm{pH}$ & Silt \% & Sand \% & Clay \% \\
\hline Mean value & 2.07 & 7.87 & 0.58 & 0.21 & 0.21 \\
Standard deviation & 0.31 & 0.11 & 0.11 & 0.14 & 0.03 \\
Minimum value & 1.54 & 7.59 & 0.38 & 0.03 & 0.14 \\
Maximum value & 3.05 & 8.15 & 0.77 & 0.48 & 0.27 \\
\hline
\end{tabular}

TOC $=$ percentage total organic carbon.

Table S2 List of the genera found that contain rhizobial species.

Bradyrhizobium
Hypomicrobium
Rhodomicrobium
Methylobacterium
Mesorhizobium
Ensifer
Rhizobium
Starkeya


Table S3. Linear mixed effect model summaries for main effects of soil abiotic factors and plant species diversity on OTU richness of bacteria, putative rhizobia, archaea, fungi, AMF, and protists.

\begin{tabular}{|c|c|c|c|c|c|c|c|}
\hline Bacteria & d.f. & $\begin{array}{l}\text { F- } \\
\text { value }\end{array}$ & $\begin{array}{l}\mathrm{p}- \\
\text { value }\end{array}$ & Fungi & d.f. & F-value & $\begin{array}{l}\mathrm{p}- \\
\text { value }\end{array}$ \\
\hline (Intercept) & 1,58 & 0.490 & 0.487 & (Intercept) & 1,56 & 0.053 & 0.818 \\
\hline $\mathrm{pH}$ & 1,58 & 0.127 & 0.723 & $\mathrm{pH}$ & 1,56 & 0.012 & 0.912 \\
\hline Sand $\%$ & 1,58 & 0.004 & 0.950 & Sand $\%$ & 1,56 & 0.089 & 0.767 \\
\hline Silt \% & 1,58 & 0.002 & 0.968 & Silt \% & 1,56 & 0.083 & 0.775 \\
\hline Clay \% & 1,58 & 0.036 & 0.850 & Clay \% & 1,56 & 0.094 & 0.760 \\
\hline TOC \% & 1,58 & 0.243 & 0.624 & TOC \% & 1,56 & 2.627 & 0.111 \\
\hline $\begin{array}{l}\text { Weed } \\
\text { cover } \%\end{array}$ & 1,58 & 0.446 & 0.507 & $\begin{array}{l}\text { Weed } \\
\text { cover } \%\end{array}$ & 1,56 & 1.760 & 0.190 \\
\hline $\log _{2}(\mathrm{SD})$ & 1,58 & 1.793 & 0.186 & $\log _{2}(\mathrm{SD})$ & 1,56 & 3.960 & 0.052 \\
\hline
\end{tabular}

\section{Rhizobia}

\begin{tabular}{llll}
\hline (Intercept) & 1,37 & 0.086 & 0.771 \\
$\mathrm{pH}$ & 1,37 & 0.791 & 0.380 \\
Sand \% & 1,37 & 0.008 & 0.929 \\
Silt \% & 1,37 & 0.005 & 0.943 \\
Clay \% & 1,37 & 0.001 & 0.976 \\
TOC \% & 1,37 & 0.390 & 0.536 \\
Weed & 1,37 & 0.491 & 0.488 \\
cover \% & 1,37 & 0.517 & 0.477 \\
Log $_{2}(\mathrm{SD})$ & 1, & &
\end{tabular}

\section{Archaea}

\begin{tabular}{|c|c|c|c|c|c|c|c|}
\hline & \\
\hline (Intercept) & 1,66 & 0.185 & 0.668 & (Intercept) & 1,55 & 1.562 & 0.217 \\
\hline $\mathrm{pH}$ & 1,66 & 0.730 & 0.396 & $\mathrm{pH}$ & 1,55 & 2.091 & 0.154 \\
\hline Sand $\%$ & 1,66 & 0.165 & 0.686 & Sand $\%$ & 1,55 & 1.243 & 0.270 \\
\hline Silt \% & 1,66 & 0.182 & 0.671 & Silt \% & 1,55 & 1.248 & 0.269 \\
\hline Clay \% & 1,66 & 0.008 & 0.927 & Clay $\%$ & 1,55 & 1.233 & 0.272 \\
\hline TOC $\%$ & 1,66 & 1.290 & 0.260 & TOC $\%$ & 1,55 & 1.679 & 0.201 \\
\hline $\begin{array}{l}\text { Weed } \\
\text { cover } \%\end{array}$ & 1,66 & 0.254 & 0.616 & $\begin{array}{l}\text { Weed } \\
\text { cover } \%\end{array}$ & 1,55 & 0.003 & 0.954 \\
\hline $\log _{2}(\mathrm{SD})$ & 1,66 & 0.524 & 0.472 & $\log _{2}(\mathrm{SD})$ & 1,55 & 0.275 & 0.602 \\
\hline
\end{tabular}

TOC, Total organic carbon.

$\log _{2}(\mathrm{SD}), \log$ plant sown diversity.

\begin{tabular}{llll} 
AMF & & & \\
\hline (Intercept) & 1,48 & 0.334 & 0.566 \\
$\mathrm{pH}$ & 1,48 & 0.444 & 0.509 \\
Sand \% & 1,48 & 0.325 & 0.572 \\
Silt \% & 1,48 & 0.331 & 0.568 \\
Clay \% & 1,48 & 0.335 & 0.565 \\
TOC \% & 1,48 & 0.213 & 0.647 \\
Weed & 1,48 & 0.071 & 0.791 \\
$\operatorname{cover} \%_{\text {og }_{2}(\mathrm{SD})}$ & 1,48 & 0.081 & 0.777 \\
\hline
\end{tabular}

\section{Protists}


Table S4. Linear mixed effect model summaries for main effects of soil abiotic factors and plant species diversity on community evenness (Simpsons' inverse, 1-D) of bacteria, putative rhizobia, archaea, fungi, AMF, and protists.

\begin{tabular}{|c|c|c|c|c|c|c|c|}
\hline Bacteria & d.f. & F-value & p-value & Fungi & d.f. & F-value & p-value \\
\hline (Intercept) & 1,58 & 1.764 & 0.189 & (Intercept) & 1,56 & 0.026 & 0.872 \\
\hline $\mathrm{pH}$ & 1,58 & 0.553 & 0.460 & $\mathrm{pH}$ & 1,56 & 0.070 & 0.793 \\
\hline Sand $\%$ & 1,58 & 0.214 & 0.646 & Sand $\%$ & 1,56 & 0.074 & 0.787 \\
\hline Silt $\%$ & 1,58 & 0.186 & 0.668 & Silt $\%$ & 1,56 & 0.078 & 0.781 \\
\hline Clay \% & 1,58 & 0.143 & 0.707 & Clay \% & 1,56 & 0.062 & 0.804 \\
\hline TOC $\%$ & 1,58 & 0.086 & 0.770 & TOC $\%$ & 1,56 & 0.281 & 0.598 \\
\hline Weed cover $\%$ & 1,58 & 0.698 & 0.407 & Weed cover $\%$ & 1,56 & 7.406 & $0.009 * *$ \\
\hline $\log _{2}(\mathrm{SD})$ & 1,58 & 0.194 & 0.661 & $\log _{2}(\mathrm{SD})$ & 1,56 & 1.704 & 0.197 \\
\hline
\end{tabular}

\section{Rhizobia}

\begin{tabular}{llll}
\hline (Intercept) & 1,37 & 0.099 & 0.755 \\
$\mathrm{pH}$ & 1,37 & 0.238 & 0.628 \\
Sand \% & 1,37 & 0.089 & 0.767 \\
Silt \% & 1,37 & 0.120 & 0.731 \\
Clay \% & 1,37 & 0.097 & 0.757 \\
TOC \% & 1,37 & 0.583 & 0.450 \\
Weed cover \% & 1,37 & 2.793 & 0.103 \\
$\log _{2}(\mathrm{SD})$ & 1,37 & 5.280 & $0.027 *$ \\
\hline
\end{tabular}

\section{Archaea}

\begin{tabular}{llll}
\hline (Intercept) & 1,66 & 0.863 & 0.356 \\
$\mathrm{pH}$ & 1,66 & 0.239 & 0.627 \\
Sand \% & 1,66 & 0.318 & 0.575 \\
Silt \% & 1,66 & 0.828 & 0.366 \\
Clay \% & 1,66 & 0.703 & 0.405 \\
TOC \% & 1,66 & 14.801 & $<.001 * * *$ \\
Weed cover \% & 1,66 & 0.279 & 0.599 \\
Log $_{2}$ (SD) & 1,66 & 0.113 & 0.738 \\
\hline
\end{tabular}

TOC, Total organic carbon.

$\log _{2}(\mathrm{SD}), \log$ plant sown diversity.

Block was specified as a random variable.

Number of asterisks denotes significance of contribution $\left(P<0.001^{* * *}, P<0.01^{* *}, P<0.05^{*}\right)$.
AMF

\begin{tabular}{llll}
\hline (Intercept) & 1,48 & 0.168 & 0.683 \\
pH & 1,48 & 0.188 & 0.667 \\
Sand \% & 1,48 & 0.184 & 0.670 \\
Silt \% & 1,48 & 0.176 & 0.677 \\
Clay \% & 1,48 & 0.198 & 0.658 \\
TOC \% & 1,48 & 0.872 & 0.355 \\
Weed cover \% & 1,48 & 2.117 & 0.152 \\
$\log _{2}(\mathrm{SD})$ & 1,48 & 1.492 & 0.228 \\
\hline
\end{tabular}

Protists

\begin{tabular}{llll}
\hline (Intercept) & 1,55 & 4.622 & 0.036 \\
$\mathrm{pH}$ & 1,55 & 1.314 & 0.257 \\
Sand \% & 1,55 & 4.197 & $0.045^{*}$ \\
Silt \% & 1,55 & 4.232 & $0.044^{*}$ \\
Clay \% & 1,55 & 4.209 & $0.045^{*}$ \\
TOC \% & 1,55 & 0.000 & 0.995 \\
Weed cover \% & 1,55 & 1.315 & 0.257 \\
$\log _{2}(\mathrm{SD})$ & 1,55 & 0.665 & 0.419 \\
\hline
\end{tabular}

$\log _{2}(\mathrm{SD})$

$1,55 \quad 0.665 \quad 0.419$ 


\section{Chapter 2}

Table S5. Linear mixed effect model summaries for main effects of soil abiotic factors and plant functional group (FG) richness on OTU richness of bacteria, putative rhizobia, archaea, fungi, AMF, and protists.

\begin{tabular}{|c|c|c|c|c|c|c|c|}
\hline Bacteria & d.f. & F-value & p-value & Fungi & d.f. & F-value & $\mathrm{p}$-value \\
\hline (Intercept) & 1,58 & 0.288 & 0.594 & (Intercept) & 1,56 & 0.437 & 0.511 \\
\hline $\mathrm{pH}$ & 1,58 & 0.150 & 0.700 & $\mathrm{pH}$ & 1,56 & 0.047 & 0.828 \\
\hline Sand $\%$ & 1,58 & 0.062 & 0.804 & Sand $\%$ & 1,56 & 0.535 & 0.468 \\
\hline Silt \% & 1,58 & 0.055 & 0.815 & Silt \% & 1,56 & 0.521 & 0.474 \\
\hline Clay $\%$ & 1,58 & 0.005 & 0.942 & Clay $\%$ & 1,56 & 0.531 & 0.469 \\
\hline TOC $\%$ & 1,58 & 0.098 & 0.755 & TOC $\%$ & 1,56 & 2.644 & 0.110 \\
\hline Weed cover $\%$ & 1,58 & 0.058 & 0.810 & Weed cover $\%$ & 1,56 & 5.259 & $0.026^{*}$ \\
\hline FG richness & 1,58 & 1.083 & 0.302 & FG richness & 1,56 & 2.396 & 0.127 \\
\hline
\end{tabular}

\begin{tabular}{llll} 
Rhizobia & & & \\
\hline (Intercept) & 1,37 & 0.216 & 0.645 \\
$\mathrm{pH}$ & 1,37 & 1.148 & 0.291 \\
Sand \% & 1,37 & 0.039 & 0.845 \\
Silt \% & 1,37 & 0.035 & 0.852 \\
Clay \% & 1,37 & 0.013 & 0.909 \\
TOC \% & 1,37 & 0.702 & 0.407 \\
Weed cover \% & 1,37 & 1.016 & 0.320 \\
FG richness & 1,37 & 2.017 & 0.164 \\
\hline
\end{tabular}

\begin{tabular}{llll} 
AMF & & & \\
\hline (Intercept) & 1,48 & 0.735 & 0.396 \\
$\mathrm{pH}$ & 1,48 & 0.497 & 0.484 \\
Sand \% & 1,48 & 0.719 & 0.401 \\
Silt \% & 1,48 & 0.730 & 0.397 \\
Clay \% & 1,48 & 0.741 & 0.394 \\
TOC \% & 1,48 & 0.075 & 0.786 \\
Weed cover \% & 1,48 & 0.271 & 0.605 \\
FG richness & 1,48 & 1.828 & 0.183 \\
\hline
\end{tabular}

\begin{tabular}{|c|c|c|c|c|c|c|c|}
\hline \multicolumn{4}{|l|}{ Archaea } & \multicolumn{4}{|l|}{ Protists } \\
\hline (Intercept) & 1,66 & 0.090 & 0.766 & (Intercept) & 1,55 & 1.094 & 0.300 \\
\hline $\mathrm{pH}$ & 1,66 & 0.722 & 0.399 & $\mathrm{pH}$ & 1,55 & 2.334 & 0.132 \\
\hline Sand $\%$ & 1,66 & 0.291 & 0.592 & Sand $\%$ & 1,55 & 0.839 & 0.364 \\
\hline Silt $\%$ & 1,66 & 0.317 & 0.576 & Silt $\%$ & 1,55 & 0.842 & 0.363 \\
\hline Clay $\%$ & 1,66 & 0.042 & 0.839 & Clay $\%$ & 1,55 & 0.835 & 0.365 \\
\hline TOC $\%$ & 1,66 & 1.004 & 0.320 & TOC $\%$ & 1,55 & 1.617 & 0.209 \\
\hline Weed cover $\%$ & 1,66 & 0.161 & 0.689 & Weed cover $\%$ & 1,55 & 0.014 & 0.907 \\
\hline FG richness & 1,66 & 2.377 & 0.128 & FG richness & 1,55 & 0.167 & 0.685 \\
\hline
\end{tabular}

TOC, Total organic carbon.

Block was specified as a random variable.

Number of asterisks denotes significance of contribution $\left(P<0.05^{*}\right)$.

Table S6. Generalised linear model summaries for main effects of soil abiotic factors, plant species diversity, and plant functional group (FG) identity on OTU richness of bacteria, putative rhizobia, archaea, fungi, AMF, and protists. 


\begin{tabular}{lclcl}
\hline Bacteria & Estimate & Std. Error & $\mathrm{z}$ value & $\operatorname{Pr}(>|\mathrm{z}|)$ \\
\hline (Intercept) & 4.405 & 1.964 & 2.243 & $0.025^{*}$ \\
$\mathrm{pH}$ & 0.134 & 0.075 & 1.799 & 0.072 \\
Sand \% & 1.140 & 1.976 & 0.577 & 0.564 \\
Silt \% & 0.984 & 1.979 & 0.497 & 0.619 \\
Clay \% & 1.622 & 2.017 & 0.804 & 0.421 \\
TOC \% & 0.011 & 0.029 & 0.367 & 0.714 \\
Weed cover \% & 0.000 & 0.000 & -0.891 & 0.373 \\
Log $(\mathrm{SD})$ & -0.003 & 0.007 & -0.360 & 0.719 \\
FG L & 0.090 & 0.027 & 3.288 & $0.001 * *$ \\
FG SH & -0.074 & 0.021 & -3.537 & $<.001 * * *$ \\
FG TH & 0.005 & 0.024 & 0.204 & 0.838
\end{tabular}

\begin{tabular}{lcccl}
\hline Fungi & Estimate & Std. Error & $\mathrm{z}$ value & $\operatorname{Pr}(>|\mathrm{z}|)$ \\
\hline (Intercept) & -22.45 & 36.65 & -0.613 & 0.540 \\
$\mathrm{pH}$ & 0.166 & 0.182 & 0.910 & 0.363 \\
Sand \% & 25.42 & 36.66 & 0.693 & 0.488 \\
Silt \% & 25.37 & 36.66 & 0.692 & 0.489 \\
Clay \% & 25.86 & 37.07 & 0.698 & 0.485 \\
TOC \% & 0.074 & 0.090 & 0.819 & 0.413 \\
Weed cover \% & 0.000 & 0.001 & -0.329 & 0.742 \\
Log 2 (SD) & 0.018 & 0.019 & 0.924 & 0.356 \\
FG L & -0.070 & 0.077 & -0.919 & 0.358 \\
FG SH & -0.088 & 0.069 & -1.279 & 0.201 \\
FG TH & 0.001 & 0.068 & 0.021 & 0.983
\end{tabular}

Null deviance: 118.594 on 28 degrees of freedom

Residual deviance: 70.659 on 18 degrees of freedom

Null deviance: 18.0357 on 27 degrees of freedom

Residual deviance: 7.3179 on 17 degrees of freedom

Rhizobia

AMF

\begin{tabular}{lcccc}
\hline (Intercept) & 24.73 & 37.66 & 0.657 & 0.511 \\
$\mathrm{pH}$ & 1.371 & 1.428 & 0.959 & 0.337 \\
Sand \% & -33.18 & 37.93 & -0.875 & 0.382 \\
Silt \% & -32.97 & 37.73 & -0.874 & 0.382 \\
Clay \% & -35.95 & 38.57 & -0.932 & 0.351 \\
TOC \% & -0.292 & 0.560 & -0.522 & 0.602 \\
Weed cover \% & 0.006 & 0.008 & 0.688 & 0.491 \\
Log $_{2}(\mathrm{SD})$ & -0.005 & 0.128 & -0.043 & 0.966 \\
FG L & 0.020 & 0.416 & 0.047 & 0.962 \\
FG SH & 0.173 & 0.358 & 0.482 & 0.630 \\
FG TH & -0.335 & 0.409 & -0.818 & 0.413
\end{tabular}

Null deviance: 2.7069 on 31 degrees of freedom

Residual deviance: 1.5547 on 21 degrees of freedom

\begin{tabular}{lcccc}
\hline (Intercept) & -59.27 & 127.80 & -0.464 & 0.643 \\
$\mathrm{pH}$ & -0.108 & 0.590 & -0.183 & 0.855 \\
Sand \% & 62.35 & 127.4 & 0.489 & 0.625 \\
Silt \% & 62.59 & 127.4 & 0.491 & 0.623 \\
Clay \% & 61.81 & 128.4 & 0.481 & 0.630 \\
TOC \% & -0.116 & 0.303 & -0.383 & 0.702 \\
Weed cover \% & 0.002 & 0.004 & 0.511 & 0.609 \\
Log $_{2}$ (SD) & 0.017 & 0.075 & 0.233 & 0.816 \\
FG L & -0.256 & 0.296 & -0.867 & 0.386 \\
FG SH & -0.060 & 0.246 & -0.242 & 0.809 \\
FG TH & 0.017 & 0.231 & 0.076 & 0.940
\end{tabular}

Null deviance: 4.3499 on 23 degrees of freedom

Residual deviance: 2.2936 on 13 degrees of freedom

Archaea

$\begin{array}{lcccc}\text { (Intercept) } & 1.425 & 15.77 & 0.090 & 0.928 \\ \text { pH } & -0.316 & 0.537 & -0.589 & 0.556 \\ \text { Sand \% } & 3.684 & 15.63 & 0.236 & 0.814 \\ \text { Silt \% } & 3.980 & 15.62 & 0.255 & 0.799 \\ \text { Clay \% } & 2.241 & 16.16 & 0.139 & 0.890 \\ \text { TOC \% } & -0.073 & 0.246 & -0.295 & 0.768 \\ \text { Weed cover \% } & 0.000 & 0.003 & -0.161 & 0.872 \\ \text { Log }_{2} \text { (SD) } & 0.009 & 0.058 & 0.162 & 0.871 \\ \text { FG L } & 0.004 & 0.224 & 0.019 & 0.985 \\ \text { FG SH } & 0.067 & 0.167 & 0.398 & 0.691 \\ \text { FG TH } & -0.048 & 0.194 & -0.250 & 0.803\end{array}$

Protists

$\begin{array}{lcccc}\text { (Intercept) } & 39.02 & 65.00 & 0.600 & 0.548 \\ \text { pH } & -0.063 & 0.296 & -0.211 & 0.833 \\ \text { Sand \% } & -35.12 & 65.09 & -0.540 & 0.590 \\ \text { Silt \% } & -35.31 & 65.05 & -0.543 & 0.587 \\ \text { Clay \% } & -34.34 & 65.99 & -0.520 & 0.603 \\ \text { TOC \% } & 0.006 & 0.199 & 0.031 & 0.975 \\ \text { Weed cover \% } & 0.000 & 0.002 & -0.119 & 0.905 \\ \text { Log }_{2}(\mathrm{SD}) & -0.009 & 0.034 & -0.258 & 0.796 \\ \text { FG L } & 0.072 & 0.128 & 0.565 & 0.572 \\ \text { FG SH } & -0.112 & 0.120 & -0.929 & 0.353 \\ \text { FG TH } & -0.049 & 0.117 & -0.422 & 0.673\end{array}$

Null deviance: 13.2306 on 27 degrees of freedom

Residual deviance: 8.8709 on 17 degrees of freedom

TOC $=$ Total organic carbon, $\log _{2}(\mathrm{SD})=\log$ plant sown diversity, $\mathrm{L}=$ legumes, $\mathrm{SH}=$ short herbs, $\mathrm{TH}=$ tall herbs .

Number of asterisks denotes significance of contribution $(P<0.001 * * *, P<0.01 * *)$. 
Chapter 2 


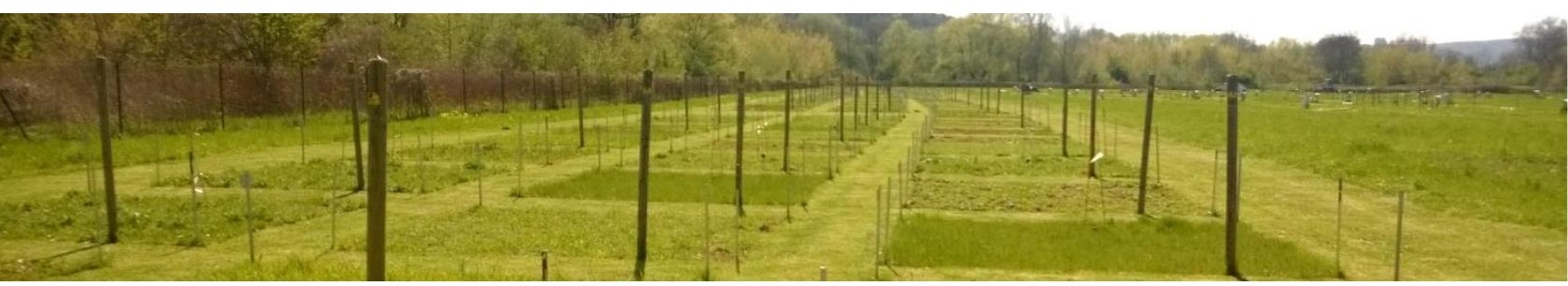

The Jena Trait-Based Experiment. 
Chapter 3

\title{
Plant traits drive root-associated fungal community composition more than plant species richness
}

\author{
Sigrid Dassen \\ S. Emilia Hannula \\ George A. Kowalchuk \\ Natalie J. Oram \\ Anne Ebeling \\ Cameron Wagg \\ Wim H. van der Putten \\ Gerlinde B. De Deyn
}




\section{Summary}

Plant performance and community composition are influenced by soil-borne fungi, yet the factors involved in the assembly of soil-borne fungi growing in and on roots (rootassociated fungi) remain unresolved.

We examined the effects of experimentally manipulated plant species richness and plant trait diversity, based on differences in rooting depth, root length density, plant height, leaf area, growth start and flowering start, on the richness and community composition of rootassociated fungi. These effects were examined in roots collected from $0-5 \mathrm{~cm}$ and $5-10 \mathrm{~cm}$ depths.

We tested the hypothesis that root-associated fungal richness positively relates to plant trait diversity. In addition, plant effects on fungal community composition were expected to be stronger in the lower root layer than in the top root layer, and we expected belowground plant traits to explain more variation in fungal community composition than aboveground or developmental plant traits.

Fungal species richness was positively related to plant species richness, but not to plant trait diversity. Fungal community composition responded significantly to plant species richness, independent of root depth layer, but the response was small compared to specific plant trait effects. The community weighted mean of root length density was the most important plant trait related to fungal community composition. Although the effect of root length density was strong in both soil layers, effects of several other plant traits differed between soil layers.

We conclude that root-associated fungal species richness increases with plant species richness, whereas root fungal community composition is driven by both plant structural and development traits. Root length density was the most important plant community trait influencing fungal community composition. 


\section{Chapter 3}

\section{Introduction}

Many fungal species live in close association with plant roots, forming both transient as well as long-lasting interactions, which can have positive, neutral or negative impacts on plant performance (Mandyam et al. 2012; Aguilar-Trigueros \& Rillig 2016). Rootassociated fungi can live on the root surface (the rhizoplane) or inside plant roots (van der Heijden \& Schlaeppi 2015; Sasse et al. 2018), and are a subset of all fungi that are present in the bulk soil (Kristin \& Miranda 2013; Goldmann et al. 2016). It is known from several grassland studies that bulk and rhizosphere soil fungal community composition is influenced, at least in part, by plant species richness and plant functional group identity (Waldrop et al. 2006; Sugiyama et al. 2008; LeBlanc et al. 2015; Dassen et al. 2017). Plants in turn can also influence the composition of root-associated fungal communities (Saks et al. 2014; Goldmann et al. 2016) by root exudation and litter production (De Boer et al. 2006; Broeckling et al. 2008). However, it remains to be tested if and how plant community trait diversity and trait identity can steer the richness and composition of rootassociated fungal communities.

In the past decade, multiple studies have reported on the fungal community compositions in bulk and rhizosphere soil of grasslands (Kowalchuk et al. 2002; Waldrop et al. 2006; Prober et al. 2015; Chen, Xu et al. 2017). However, there have been very few studies on root-associated fungi in grasslands, and these have been predominantly focussed on mycorrhizal colonisation. Recently, it has been shown in an experimental grassland that increasing plant species richness from monocultures to eight-species mixtures has no effect on overall root-associated fungal species richness, although the number of plant pathogenic fungi was higher in monocultures than in eight-species mixtures (Mommer et al. 2018). The authors suggested that the shift in fungal composition was non-random, because hostspecific pathogens are lost or diluted in plant mixtures, potentially explaining why plant species mixtures produce more biomass than monocultures (Maron et al. 2011; Schnitzer et al. 2011).

Other root-associated fungi that can influence the performance of plant individuals and communities include arbuscular mycorrhizal fungi (AMF) and other fungal root endophytes (Clay 1999; Aguilar-Trigueros \& Rillig 2016; Geisen et al. 2017). To date, it is unknown whether fungal root endophyte richness and community composition are influenced by plant species richness, and if plant traits could be used to predict changes in fungal root endophyte communities. There is clear evidence that plant functional group identity 
impacts root-associated fungal community composition; in general, grasses typically support higher root endophyte abundance and diversity than forbs (Weishampel \& Bedford 2006; Mandyam et al. 2012), whereas forbs tend to host more abundant and more diverse AMF communities in their roots than grasses (Wilson \& Hartnett 1998; Cortois et al. 2016; Dassen et al. 2017).

Plant species within the same functional group can also vary in their root-associated fungal community composition and fungal species richness (Wearn et al. 2012; Wehner et al. 2014). Therefore, root samples from a mixed plant community can be expected to host greater fungal species richness than roots from monocultures. Nevertheless, in studies on plant species richness effects on fungal communities in soil and the rhizosphere typically a large variation in fungal species richness is found within a given level of plant species richness (Sugiyama et al. 2008; Dassen et al. 2017; Yang et al. 2017). Other plant community characteristics might partially explain this variation, for example, plant trait diversity (Wardle 2004; Legay et al. 2014; Leff et al. 2018) or the phylogenetic relatedness of plants (Wehner et al. 2014). For example, phylogenetically conserved plant traits, such as specific root length of grassland plant species, have been shown to be a good predictor of AMF colonisation (Cortois et al. 2016). It is therefore expected that besides plant species richness, trait diversity also can partially explain the richness of root-associated fungal communities.

It has been suggested that belowground plant traits can explain the variation in soil microbial community composition in grasslands better than aboveground traits (Freschet et al. 2013). Root traits might reflect soil parameters, and they also might determine the quality and quantity of root exudates that provide resources to root-associated microorganisms (Legay et al. 2014). Root exudates are known to have a strong influence on bulk soil fungal community composition (Broeckling et al. 2008), as well on AMF colonisation (Vierheilig et al. 2003; Steinkellner et al. 2007), yet the soil fungal community can also respond to aboveground plant traits as aboveground plant material eventually becomes a food source for soil fungi when it enters the soil as litter (Cortez et al. 2007; Cornwell et al. 2008; Liu et al. 2018). The disruption of removing aboveground biomass by mowing grasslands can limit the litter accumulation process, and thus the effects of aboveground plant traits on soil fungal communities are expected to also relatively weak. Traits related to plant development, such as the onset of growth and flowering, potentially influence root-associated and soil fungal communities via the timing and composition of released root exudates and/or litter (Luster et al. 2009; Lauber et al. 2013). 


\section{Chapter 3}

Rooting depth is influenced by both plant identity (Bardgett et al. 2014) and the vertical gradient in soil fertility (Fierer et al. 2003; Delgado-Baquerizo et al. 2017), and fungal community composition may respond to such vertical stratification of plant roots. For example, bulk soil fungal species richness and abundance have been shown to decline with increasing soil depth (Fierer et al. 2003; Sanaullah et al. 2016), and fungal community composition also changes with depth (Jumpponen et al. 2010; Moll et al. 2016; Sanaullah et al. 2016). Plant community diversity effects on root-associated fungi might, therefore, differ between soil layers. In addition, saprophytes comprise a large proportion of the soil fungal community, and this group is assumed to be composed of more generalists than AMF and plant pathogens. The saprophyte community is probably most active (and most abundant) in the upper soil layer and a large group of generalists could overrule plant diversity effects on root-associated fungi.

The aim of the present study was to analyse effects of plant species richness and plant trait diversity on root-associated fungal richness and community composition at two rooting depths. We tested the hypotheses that (1) root-associated fungal richness is positively related to plant species richness and plant trait diversity (2) plant species and trait diversity effects explain variation in the root-associated fungal community composition in the lower root layer $(5-10 \mathrm{~cm})$ more than in the upper layer $(0-5 \mathrm{~cm})$, and (3) root-associated fungal community composition is better explained by belowground than by aboveground plant structural traits or plant development traits.

To test these hypotheses, root samples were collected from the Trait-Based Experiment in Jena, Germany (Ebeling et al. 2014). At this grassland site, plant species richness and trait diversity have been experimentally manipulated. Root-associated fungal richness and community composition was examined by high-throughput sequencing of root samples, and related to plant species richness, trait diversity, and specific traits.

\section{Materials and methods}

In August 2014, root samples were taken in the Trait-Based Experiment in Jena (Germany). The Trait-Based Experiment is located on the field site of the Jena Experiment and was setup in 2010 and re-sown in 2011 (Ebeling et al. 2014). The established plant communities have different levels of trait complementarity, with functional diversity ( $\left.\mathrm{FD}_{\mathrm{Jena}}\right)$ ranging from plant communities with similar plant traits $\left(\mathrm{FD}_{\text {Jena }} 1\right)$ to plant communities with high trait complementarity $\left(\mathrm{FD}_{\text {Jena }} 4\right)$. The plant structural traits that were used to design the 
experiment were: maximal rooting depth $(\mathrm{m})$, root length density $\left(\mathrm{cm} \mathrm{root} / \mathrm{cm}^{3}\right.$ soil), plant height $(\mathrm{cm})$ and leaf area $\left(\mathrm{cm}^{2}\right)$, the plant development traits are: growth start (week of the year) and flowering start (cumulative growing degree days in ${ }^{\circ} \mathrm{C}$ ). From all non-legume plant species used in the Jena Experiment (Roscher et al. 2004) those six traits were measured and the results were analysed by principal component analysis. Along the first axis plant species are separated according to plant structural traits, whereas along the second axis plant species are separated according to plant development traits. The plant species along the first and second axis are grouped into pools (pool 1 and 2, respectively). In each pool, a few plant species have traits that overlap and are therefore present in both pools (Ebeling et al. 2014; Steinauer et al. 2017)(Appendix Fig 1).

Each pool was subdivided into 4 evenly spaced sectors, and within each sector two plant species were chosen to represent the sector of the particular pool (Appendix Table 1). The distance between the sectors determines the level of plant community functional diversity $\left(\mathrm{FD}_{\text {Jena }}\right)$. For example, a plant community with plant species from the same sector had $\mathrm{FD}_{\text {Jena }} 1$, and a plant community with plant species from two neighbouring sectors had $\mathrm{FD}_{\mathrm{Jena}}=2$. Plant communities were monocultures, 2, 3, 4, or 8 species mixtures, the combinations of $\mathrm{FD}_{\mathrm{Jena}}$ levels and plant species richness are shown in Appendix Table 2. The experiment has 3 blocks. Each block is a replication of the functional diversity gradient and plant species richness gradient, whereby plant identities within each plant community at each level of plant species and functional diversity vary among the blocks in order to avoid sampling effects, for further details on the design see Ebeling et al. (2014). The plots in the Jena Experiment are mown twice a year following the biomass harvest and are hand weeded three times a year to remove non-sown plant species.

Root samples were collected from 92 plots, using a corer of $4 \mathrm{~cm}$ diameter to sample 0-10 $\mathrm{cm}$ soil cores. Per plot 8 cores were taken, divided into 0-5 and 5-10 $\mathrm{cm}$ depth, and pooled per depth. Cores were stored at $4{ }^{\circ} \mathrm{C}$ until washed over a $0.5 \mathrm{~mm}$ sieve (within 32 hours), and per depth layer a sample of $50 \mathrm{mg}$ clean roots was stored at $-80{ }^{\circ} \mathrm{C}$ until DNA extraction.

DNA was extracted from $50 \mathrm{mg}$ roots with the DNaesy 96 Plant mini kit (Qiagen, Venlo, The Netherlands). Root sampling and DNA isolation was part of a joint sampling campaign with Oram et al. (2018). The fungal ITS2 region was amplified with primer pair ITS4/ITS9 (Ihrmark 2012) with 12 base pair multiplex identifier tags on the primers. Amplicons were purified with Agencourt AMPure XP (Beckman Coulter, Brea, US) protocol according to 


\section{Chapter 3}

the manufactures protocol. Subsequently, purified amplicons were quantified with Standard Sensitivity NGS Fragment Analysis kit (Advanced Analytical, Ankeny, US). Equal molar amounts on each sample were pooled, and send to BGI (Hong Kong) for Illumina Miseq PE 250 sequencing.

Sequences were analysed using the PIPITS pipeline (Gweon et al. 2015). To summarise the process, raw sequences were paired and quality filtered with the FASTX-Toolkit, ITS2 regions were extracted with ITSx, singletons removed and then clustered into OTUs with VSEARCH at $97 \%$ sequence similarity, removal of chimeras was done with UNITE UCHIME and then taxonomy was assigned to the sequences with the RDP Classifier against the UNITE fungal ITS database. In FUNGuild (Nguyen et al. 2016) OTUs were classified to potential function. Those OTUs that could be classified were grouped into saprophytes, AMF, plant pathogens (at species level, checked with literature), endophytes, and rest (EM, fungal/animal/unidentified plant pathogens).

\section{Data analysis}

The effect of plant species richness and trait diversity on fungal species richness was analysed using a linear mixed model with plant species richness (log transformed), plant trait diversity ( $\mathrm{FD}_{\mathrm{Jena}}$, , soil layer $(0-5,5-10 \mathrm{~cm})$, and plant species pool, as fixed factors and block as random factor Sown plant species richness was added into the model first and then plant trait diversity, because the levels of plant species richness and trait diversity are not fully factorial (Appendix Table 2). In this way the effect of plant trait diversity is tested after accounting for the effect of monocultures and eight plant species mixtures. In the model, there was no interaction of sown plant species richness and plant trait diversity.

To test whether the effects of plant trait diversity, sown plant species richness, and plant pool were different between the two root layers, distance-based redundancy analyses (dbRDA) with forward selection of explanatory plant community variables were used. The fungal communities of the two root layers were analysed separately in order to obtain separate information for each layer on the effect size of the explanatory variables on the fungal community composition. The fungal relative abundance data were normalised by centred log ratio transformation and community distances were calculated with Hellinger transformation. Block was added as co-variate, and explanatory variables were included into the model if $\mathrm{P}_{\mathrm{adj}}<0.05$. 
The effect of plant structural and development traits on fungal root community composition was tested for both root layers separately by db-RDA with forward selection. Plant pool and the community-weighted mean of plant height, leaf area, rooting depth, root length density, growth start and flowering start were included into the model if $\mathrm{P}_{\text {adj }}<0.05$. Block was added as co-variate.

To test whether the relative abundances of fungal functional groups differed between the two root layers mixed models were used. Relative abundance of saprophytes, AMF and the rest group was log transformed, while root layer was considered as a fixed factor and block a random factor. The relative abundance data of plant pathogens and endophytes contained many zeros. We therefore performed a two-step analysis, in which we first tested whether the occurrence of plant pathogens or endophytes was different between the two root layers (chi-square test). Secondly, all observation in which no pathogens or endophytes were detected were removed from the dataset and subsequently we tested with a linear model whether the average relative abundance of plant pathogens and endophytes differed between the two root layers.

Community weighted mean of the specific plant traits (root length density, rooting depth, leaf area, plant height, growth start, and flowering start) was calculated by using the species specific aboveground plant biomass. We used a mixed model to assess whether the community weighted mean of the six plant traits could explain the abundance of AMF and saprophytes. Relative abundance of the fungal groups was log transformed, layer and the six plant traits (no interactions among the plant traits) were considered as fixed factors and block as a random factor.

The linear models and mixed models were performed in $\mathrm{R}$ with package nlme (Pinheiro 2018), the db-RDAs were made in CANOCO version 5.0. Figures were made in $\mathrm{R}$ with package 'ggplot' (Wickham 2009) or CANOCO and further edited in Adobe Illustrator.

\section{Results}

In total, 4119 fungal OTUs were identified from 3,675,587 high quality reads. Among the 4119 OTUs, about $20 \%$ could be classified into one of the potential functional groups by FUNGuild (Appendix Fig. 2). The most abundant and species rich group was the sapropytes, with 604 OTUs. Thereafter, 586 OTUs were taxonomically identified as AMF. A total of 15 OTUs that could be classified to the species level were identified as potential plant pathogens based upon pathogenicity information available in the literature (Appendix 


\section{Chapter 3}

Table 3). However, we could not detect identifiable potential plant pathogens in the majority of samples, and the maximum number of potential pathogenic OTUs per sample was four. We further detected 28 OTUs classified as non-mycorrhizal root endophytes of which one was a potential plant pathogen according to literature. Because each sample had low numbers of OTUs that could be classified as root-associated plant pathogens and nonmycorrhizal root endophytes, we did not separately analyse these two groups of rootassociated fungi. The remainder of OTUs that could be classified to potential functional groups included ectomycorrhizae and fungal/animal/unidentified plant pathogens.

\section{Root-associated fungal OTU richness}

Total root-associated fungal OTU richness increased with plant species richness $\left(\mathrm{F}_{1,154}=\right.$ 9.00, $\mathrm{P}=0.0027$ ), and this effect was observed for both root layers (plant species richness $\mathrm{x}$ root layer $F_{1,154}=0.28, P=0.60$ ) (Fig. 1). In contrast, root-associated fungal richness did not increase with increasing plant trait diversity $\left(\mathrm{F}_{1,154}=0.04, \mathrm{P}=0.84\right)$, and this was independent of root layer (plant trait diversity $\mathrm{x}$ root layer $\mathrm{F}_{1,154}=0.23, \mathrm{P}=0.63$ ). The average number of root-associated fungal OTUs in the $0-5 \mathrm{~cm}$ layer was $527 \pm 11 \mathrm{SE}$ and $481 \pm 11 \mathrm{SE}$ in the 5-10 cm layer. No significant difference in fungal OTU richness was observed between the two plant pools. To check for consistency of the results, we also used the Chao1 index and rarefied OTU richness as diversity indices, and the results of both indices were in line with the results for observed OTU richness (data not shown). None of the six plant specific traits were significantly related to root-associated fungal richness (data not shown).

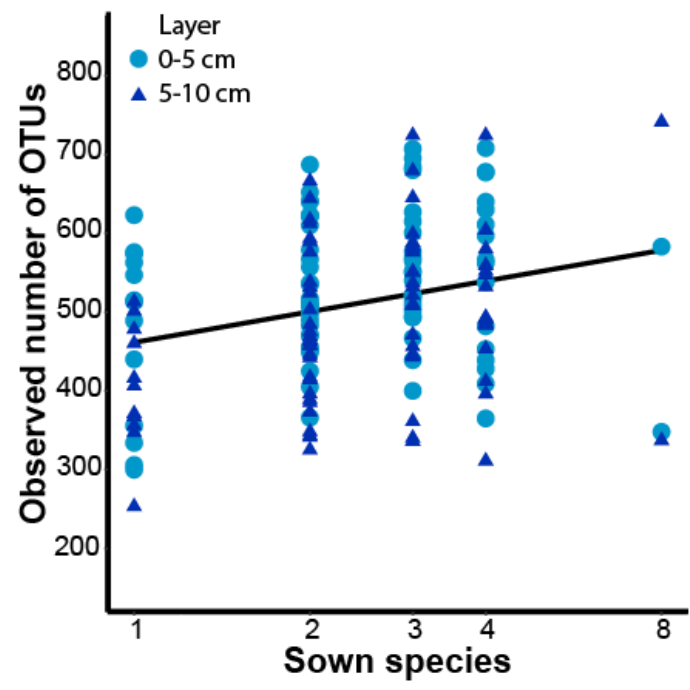


Figure 1 Observed fungal OTU richness in plant roots of 0-5 cm (circles) and 5-10 cm depth (triangles) across a plant species richness gradient.

We found that saprophyte OTU richness increased with plant species richness $\left(\mathrm{F}_{1,154}=\right.$ $5.25, \mathrm{P}=0.022)$ and that saprophyte OTU richness was higher in the $0-5 \mathrm{~cm}$ layer $(82 \pm 2$ $\mathrm{SE})$ than in the $5-10 \mathrm{~cm}$ layer $(70 \pm 2 \mathrm{SE})\left(\mathrm{F}_{1,154}=21.03, \mathrm{P}<0.001\right)$. On the other hand, there was no significant relation between AMF OTU richness and plant species richness $\left(\mathrm{F}_{1,154}=3.69, \mathrm{P}=0.0548\right)$ or depth layer $\left(\mathrm{F}_{1,154}=0.0007, \mathrm{P}=0.98\right)$.

\section{Root-associated fungal community composition}

In each root layer, the community composition of the root-associated fungi was significantly related to plant species richness $\left(0-5 \mathrm{~cm}\right.$ : pseudo-F $=1.5, \mathrm{P}_{\text {adj }}=0.048 ; 5-10$ $\mathrm{cm}$ : pseudo- $\mathrm{F}=1.5, \mathrm{P}_{\mathrm{adj}}=0.032$ ), with similar levels of explained variation, $1.7 \%$ and $1.8 \%$ respectively (Fig 2). There was no significant effect of plant trait diversity on rootassociated fungal community composition in none of the two root layers. The factor that influenced the fungal communities most was plant species pool. The total amount of variation explained by plant species pool and plant species richness in root-associated fungal community composition was $3.6 \%$ in the top root layer and $4.1 \%$ in the lower layer.

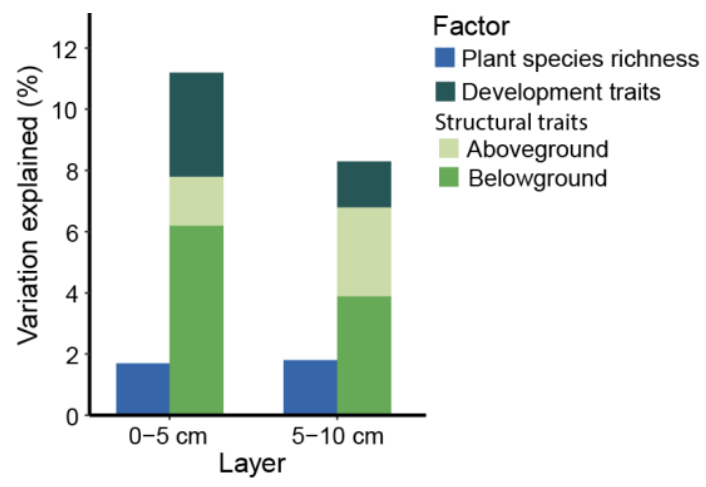

Figure 2 Overview of variation explained in fungal community composition by plant factors from the db-RDA analyses. The figure sums up the plant traits that significantly contributed to fungal community composition. Plant development traits are growth start and flowering start, aboveground plant traits are leaf area and plant height, and belowground plant traits are rooting depth and root length density.

Root length density was the plant trait that exerted the greatest influenced on the community composition of root-associated fungi in both the top and lower root layers (0-5 $\mathrm{cm}$ : variation explained: $4.6 \%$, pseudo-F $=3.9, \mathrm{P}_{\mathrm{adj}}=0.002 ; 5-10 \mathrm{~cm}$ : variation explained: 


\section{Chapter 3}

$3.9 \%$, pseudo-F $\left.=3.4, \mathrm{P}_{\mathrm{adj}}=0.002\right)$ (Fig 3). Other plant traits that influenced rootassociated fungal community composition had inconsistent effects between the two root layers. In the top layer, plant development traits (flowering start and growth start) explained some variation in root-associated fungal communities, whereas in the lower root layer the aboveground structural traits (mean plant height and leaf area) were contributors. The total amount of variation explained by plant traits and plant species pool in root-associated fungal community composition was $13 \%$ in the top soil layer and $10.4 \%$ in the lower layer.
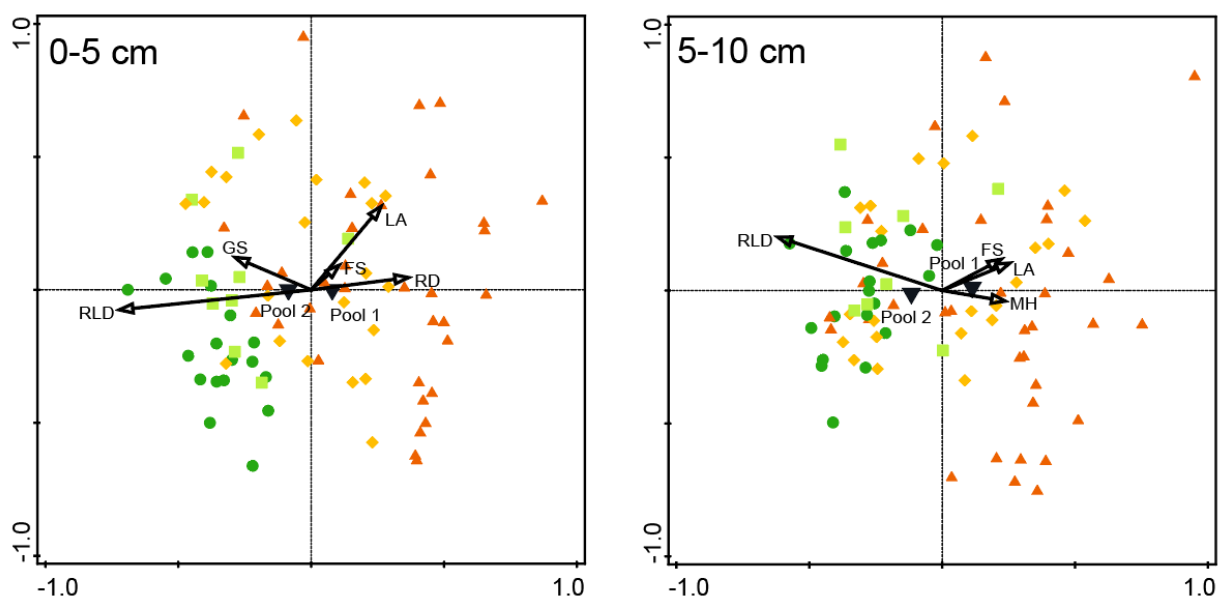

$$
\begin{aligned}
& >75 \% \text { grass } \\
& >50 \% \text { grass } \\
& >>50 \% \text { forb } \\
& >>75 \% \text { forb }
\end{aligned}
$$

Figure 3 PCA of root fungal community composition in two root layers (0-5 and 5-10 cm depth). The plant traits shown are significantly $(\mathrm{P}<0.05)$ contributing to the model and were tested by db-RDA with forward selection. The factors in the $0-5 \mathrm{~cm}$ layer explain $13 \%$ of variation in root fungal community composition, in the $5-10 \mathrm{~cm}$ layer the factors explain $10.4 \%$ of the variation. $\mathrm{RLD}=$ root length density, $\mathrm{RD}=$ rooting depth, GS = growth start, FS = flowering start, LA = leaf area. The colour gradient represents the cover percentage of grasses and forbs in the plot from which the sample was taken: dark green circle $>75 \%$ of grass cover, light green square $>50 \%$ grass cover, orange diamond $>50 \%$ forb cover, and dark orange triangle $>75 \%$ forb cover.

\section{Fungal functional groups}

The relative abundance of saprophytes was greater in the top layer $(0.157 \pm 0.010)$ than in the lower layer $(0.123 \pm 0.008)\left(F_{1,164}=11.31, P=0.001\right)$ (Appendix Fig 2). Relative abundance of AMF was neither significantly different between the top $(0.043 \pm 0.004)$ and lower layer $(0.049 \pm 0.004)\left(F_{1,164}=1.77, P=0.19\right)$, nor was the occurrence or relative 
abundance of plant pathogens different between the two root layers (occurrence $48.2 \%$ and $35.3 \%$ in the upper and lower layer respectively, $X^{2}=2.37$, df $=1, p=0.12$; relative abundance: $\left.F_{1,68}=0.0973, P=0.76\right)$. Endophytes were more represented in samples from the top layer than the lower layer $\left(78.3 \%\right.$ and $55.3 \%$ respectively) $\left(X^{2}=9.00\right.$, df $=1, p=$ 0.0027 ), whereas they were relatively more abundant in the fungal community of the lower layer $(0.0015 \pm 0.0010)$ than in the upper layer $(0.0010 \pm 0.0003)\left(\mathrm{F}_{1,110}=8.46, \mathrm{P}=\right.$ $0.0044)$.

Overall, saprophytes and AMF were the two most prominent fungal functional groups identified in the roots (Appendix Fig1). Therefore, we tested how the community weighted means of the six plant functional traits were related to the relative abundance of saprophytes and AMF. The relative abundance of saprophytes was negatively related to the growth start (week of the year) of the plants in the community $\left(F_{1,152}=5.219, P=0.024, r=\right.$ -1.29) (Fig 4a). Root length density of the plant community related negatively with AMF relative abundance $\left(F_{1,152}=12.89, P=0.0004, r=-0.33\right)(F i g 4 b)$. The effects of plant traits on the relative abundance of saprophytes or AMF were independent of root layer depth.
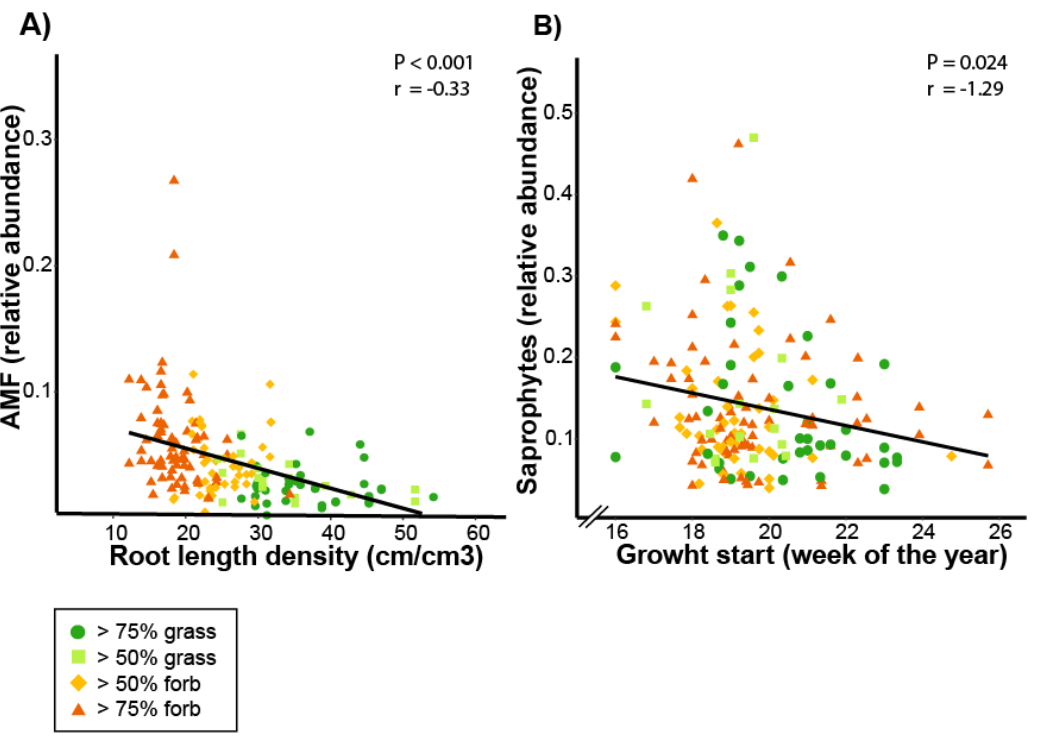

Figure 4 Relation (a) between root length density and relative abundance of AMF in the plant roots and (b) between the plant development trait growth start and relative abundance of saprophytes in the plant roots. The colour gradient represents the cover percentage of grasses and forbs in the plot from which the sample was taken: dark green circle $>75 \%$ of grass cover, light green square $>50 \%$ grass cover, orange diamond $>50 \%$ forb cover, and dark orange triangle $>75 \%$ forb cover. 


\section{Discussion}

We assessed the effects of plant species richness and plant trait diversity on the richness and composition of root-associated fungi. In support of our hypothesis (1) that the richness of root-associated fungi should increase with plant species richness and plant trait diversity, we found that increasing plant species richness resulted in greater fungal richness. However, plant functional trait diversity was not related to fungal richness. Subsequently, we tested the hypothesis that plant species richness and trait diversity would explain more variation in the root-associated fungal community composition from the lower root layer $(5-10 \mathrm{~cm})$ than from the upper layer $(0-5 \mathrm{~cm})$. Contrary to our hypothesis, there was no interaction effect of root layer and plant species richness, or trait diversity, on rootassociated fungal richness. However, roots in the $0-5 \mathrm{~cm}$ layer contained more fungal OTUs than the 5-10 cm layer. This differentiation of fungal richness can have biotic and abiotic causes, for example, different levels of resources or soil moisture. Our experimental approach does not allow to identify the exact cause of the differentiation. We also found that plant trait diversity and specific plant traits had no significant effect on overall fungal richness. However, fungal community composition and relative abundance of specific fungal functional groups responded to several plant traits, with root length density being the most important plant trait associated with fungal community composition. These findings are partially in support of our hypothesis (3) that root-associated fungal community composition is better explained by belowground than by aboveground plant structural traits or plant development traits.

\section{Plant community diversity and trait effects on root-associated fungal species richness}

We hypothesised that root-associated fungal richness would be positively related to both plant species richness and plant trait diversity. This hypothesis was only partially supported, as we found root-associated fungal richness to be positively related to plant richness, but not to plant trait diversity. Our findings differ from Mommer et al. (2018) who found no significant effect of plant species richness on overall root-associated fungal richness. However, they found a similar positive relation for a sub-set of the fungal community, with fungal endophyte richness being positively related to plant species 
richness. The analysis of Mommer et al. (2018) was performed using a similar gradient of plant species richness (1-8 species) and the plant species identities partly overlapped with our plant species. Possible underlying causes for the contrasting findings could be the soil type on which the experiments were performed; the experiment of Mommer et al. (2018) was performed on sandy soil, whereas the Jena Experiment is set up on loamy soil. These soils also differ somewhat in $\mathrm{pH}$ (averages of 7.9 and 7.3 for the Jena Experiment and Wageningen Biodiversity Experiment, respectively) and nutrient levels. These factors that are known to be important determinants of soil microbial community composition (Rousk et al. 2010; Sun et al. 2016). Root-associated fungal community composition can also change through time (Mandyam \& Jumpponen 2008). Thus, the length of time between the establishment of the experiments and sampling of the roots might also impact these differential findings. The Jena Trait-Based Experiment was sampled 3 years after its establishment, whereas the Wageningen Biodiversity Experiment was sampled 10 years after establishment. To our knowledge, there are no other studies that have focussed on plant diversity effects on root-associated fungal communities studied by next generation sequencing, so that more studies are needed in order to detect general patterns.

In our study, besides overall fungal richness, saprophyte richness was also positively related to plant species richness, which was unexpected given that saprophytes are mostly considered to be generalists (Leff et al. 2018). Possibly, root-associated saprophytes are more specialised or are under stronger influence of plants than saprophytes in the bulk soil. It is also possible that root-associated saprophytes respond to an increase in root biomass with plant species richness (Oram et al. 2018), which subsequently results in greater amounts of root litter deposition and root exudation upon which saprophytes feed (Hannula et al. 2012). In contrast, we did not detect a relationship between AMF richness and aboveground plant species richness, which is in line with Öpik et al. (2008), but in contrast to Hiiesalu et al. (2014) who found a positive relation between AMF species richness and belowground plant species richness.

We expected that the variation in fungal richness within each level of plant species richness may be explained at least in part by plant trait diversity. However, plant trait diversity had no significant effect on fungal richness. Even among monocultures, there was substantial variability in fungal richness. In addition, overall root-associated fungal richness was not significantly affected by the six plant traits tested. However, we found a strong negative correlation between AMF relative abundance and root length density. These results might indicate that plant traits and plant trait diversity are poor predictors of overall fungal 


\section{Chapter 3}

richness (Barberán et al. 2015; Leff et al. 2018), but that specific traits can correlate to specific microbial activity (Cantarel et al. 2015) or the abundance of specific fungal functional groups (Cortois et al. 2016).

\section{Root depth effects on root-associated fungal community richness and composition}

We observed that root-associated fungal species richness was higher in the top than in the lower layer, which is in line with other studies (Fierer et al. 2003; Jumpponen et al. 2010). In general, saprophytes comprised a large part of the root-associated fungal community. We assumed a larger density of organic matter in the top than lower soil layer (Steinbeiss et al. 2008; Ravenek et al. 2014) and a generalist behaviour of soil and plant-associated fungal saprophytes. We further assumed that a large group of generalists could overrule any plant diversity effects, and we therefore expected that plant community diversity effects would explain more variation in the root-associated fungal community composition in the deeper root layer. Contrary to our second hypothesis, the strength of the relation between plant species richness and fungal richness was similar in the two soil layers. In addition, plant species richness and trait diversity effects on root-associated fungal community composition did not differ between the two root layers either. Weeding and mowing activities at the Jena Trail-Based Experiment will reduce the aboveground litter input, which may limit the build-up of a distinct litter layer. However, root biomass comprises of up to $70 \%$ of total biomass in grassland systems (Poorter et al. 2011), and in the Jena trait based experiment, there is more root biomass in the upper $5 \mathrm{~cm}$ than in the $5-10 \mathrm{~cm}$ layer (Oram et al. 2018). Thus, this densely rooted layer likely produces large amounts of rootderived litter. It was also observed that the fungal community composition, analysed by distance-based RDA's, did not parallel the observed difference in fungal richness between the layers. This is probably a result of the fact that changes in species richness in distancebased RDA's generally have less power than changes in species relative abundance.

\section{Plant trait effects on root-associated fungal community composition}

In the upper soil layer, and to a lesser extent in the lower soil layer, root-associated fungal community composition was well correlated to percentage cover of grasses and forbs. Even though plant functional groupings have been based on common traits (Roscher et al. 2004), there was a relatively large degree of variation in specific traits within plant functional groups. Root-associated fungal community composition was expected to be explained more 
by belowground traits than by aboveground plant structural and development traits. In support of this hypothesis, we found that root length density was the plant trait that best explained root-associated fungal community composition. We also found a negative correlation between root length density community weighted mean and AMF relative abundance, which was accompanied by a gradient of samples taken from forb-dominated plots to grass-dominated plots. Root length density is a measure of the amount of fine roots, and it correlates well with the relative abundance of grass root biomass per sample (Oram et al. 2018). Plants with fine roots are generally better at extracting nutrients and water from soil than plants with coarse roots, and they are therefore less dependent on mycorrhizal associations (Smith \& Read 2008b; Ma et al. 2018). However, the assumption that coarserooted plants depend more on AMF for capturing soil resources is not supported by all studies (Maherali 2014).

Aboveground plant development traits also explained root-associated fungal community composition depending on root layer. In the top layer, plant development traits had a relatively large contribution to fungal community composition. We also found that the community weighted mean of growth start and root saprophyte relative abundance were negatively correlated. These results may be explained by the fact that plant development affects root age and activity, which also affects the amount and composition of rhizodeposition (Sasse et al. 2018). Early growth could mean that roots release root exudates for a relative long period and that these large amounts of exudates together with the turnover of dead roots may cause accumulation of fungal saprophytes. It has also been shown that, aside from root exudates, decomposing plant roots are a carbon source for saprophytic fungi (Hannula et al. 2012). Larger amounts of dead plant roots in the top layer could be related to the differential root-associated fungal community composition between the two depth layers.

The relation between aboveground plant traits and root-associated fungal community composition was most prominent in the lower root layer. This is perhaps a bit surprising, as we expected that root-associated fungi are unlikely to be directly influenced by aboveground plant traits such as leaf area and plant height. However, plant traits can predict resistance to herbivory (Carmona et al. 2011) and, in turn, foliar herbivores can induce changes in root exudation and litter quality, which subsequently impact root-associated microbes (Pangesti et al. 2013). Other possible relations between root-associated fungi and aboveground plant traits include litter quality, effects on soil moisture or soil depthdependent root exudation differences. 


\section{Chapter 3}

A maximum of $13 \%$ of the variation in root-associated fungal community composition could be explained by specific plant traits. Few studies on root-associated fungi included plant community composition as a factor in the analysis. However, in beech forests, $3.8 \%$ of the variation in root-associated fungal communities could be explained by plant community composition, whereas soil chemical and texture properties together explained $38 \%$ of the variation (Goldmann et al. 2016). The finding that plant community composition has relative smaller effects on fungal root-associated community composition than other (abiotic) factors could indicate that at least the more abundant root-associated fungi are rather generalists.

\section{Conclusions}

We show that that root-associated fungal community composition is influenced more by plant structural and development traits than by plant species richness. Moreover, our study supports previous findings that greater plant richness can support a greater richness of belowground diversity, here with specific focus on root-associated fungi. However, plant trait diversity does not seem to be a significant determinant of root-associated fungal community composition or richness. Therefore, our results indicate that the assembly of root-associated fungi in plant communities is influenced more by plant-specific traits than by the diversity of traits that are present in the plant community. Our results indicate that an important new research question will be to understand which specific plant traits influence the assembly of root-associated fungal communities. 


\section{Supporting information}

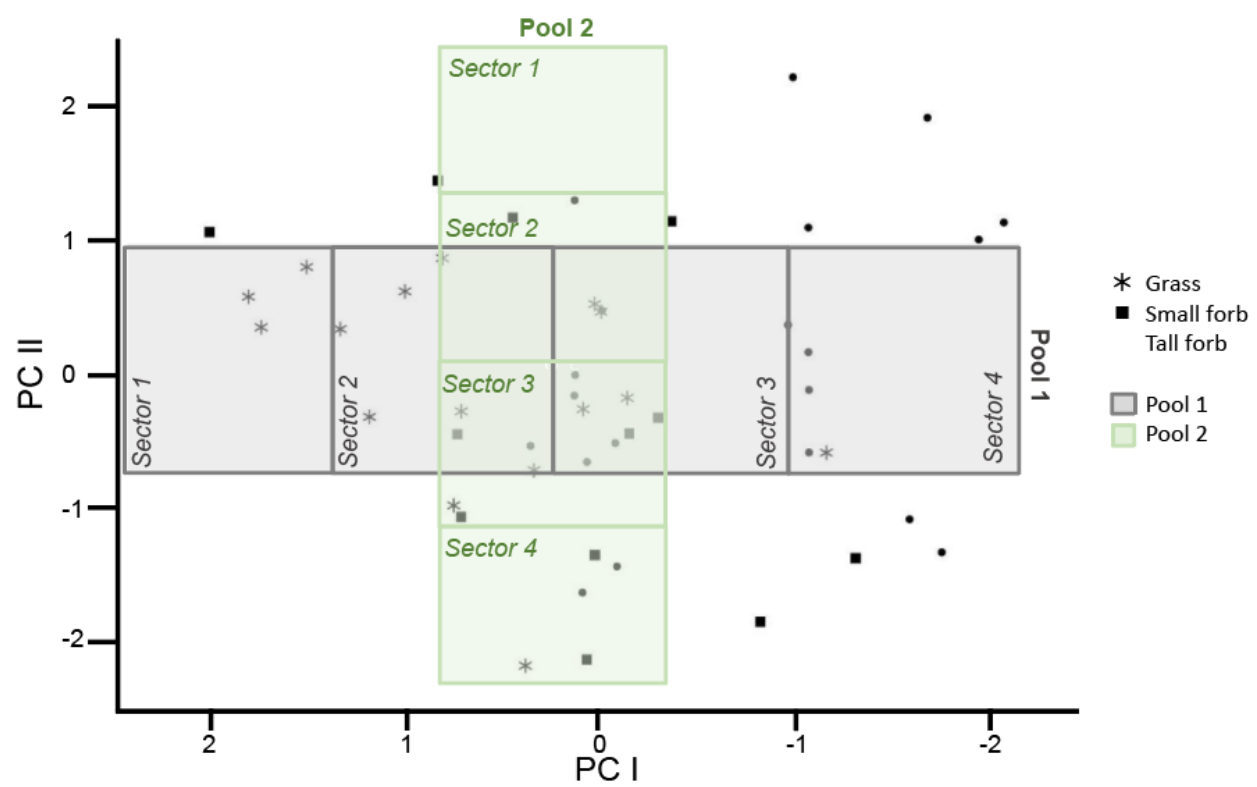

Appendix Fig 1 Principal component analysis of six plant traits (maximal rooting depth, root length density, plant height and leaf area, growth start, and flowering start). Plant structural traits are maximised on the $\mathrm{x}$-axis (Pool 1) and plant development traits are maximised on the y-axis (Pool 2). Within each sector two plant species are representative for the sector of the particular pool. The distance between the sectors determines the level of plant community functional diversity ( $\left.\mathrm{FD}_{\mathrm{Jen}}\right)$. The figure is modified after Ebeling et al. (2014) and Steinauer et al. (2017). 


\section{Chapter 3}

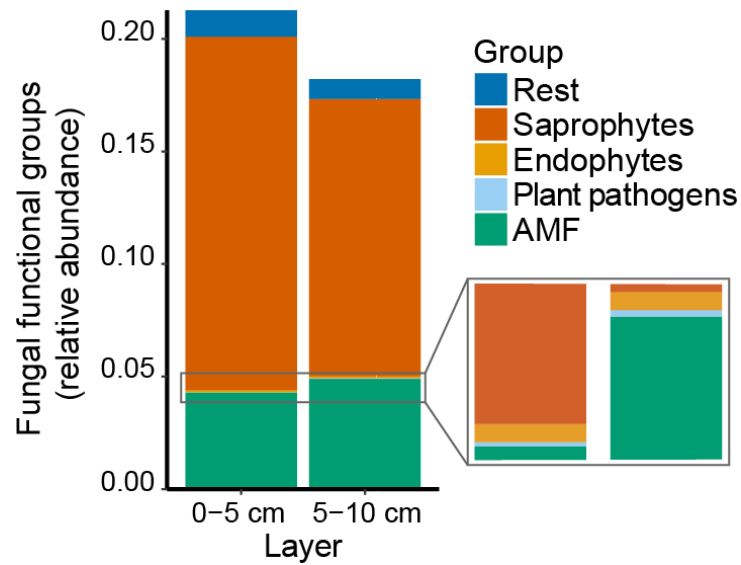

Appendix Fig 2 Relative abundance of fungal functional groups in the 0-5 cm and 5-10 cm layer. Of the 4119 fungal OTUs about $20 \%$ could be classified into one of the potential functional groups by FUNGuild. Potential plant pathogens are at species level and checked with literature, the rest groups includes ectomycorrhizae and fungal/animal/unidentified plant pathogens. 
Appendix table 1 Selected plant species per plant pool per sector, see also appendix figure 1.

\begin{tabular}{lllll}
\hline \multirow{2}{*}{ Pool } & Sector 1 & Sector 2 & Sector 3 & Sector 4 \\
\hline \multirow{2}{*}{1} & Festuca rubra & Avenula pubescens & Leucanthemum vulgare & Centaurea jacea \\
& Poa pratensis & Phleum pratense & Plantago lanceolata & Knautia arvensis \\
\multirow{2}{*}{2} & Holcus lanatus & Phleum pratense & Dactylis glomerata & Anthoxanthum odoratum \\
& Geranium pratense & Plantago lanceolata & Leucanthemum vulgare & Ranunculus arcis \\
\hline
\end{tabular}

Appendix table 2 Overview of the number of replicates of each plant species richness and functional diversity (FD Jena) level.

\begin{tabular}{llllll}
\hline Plant species richness & FD Jena $_{1}$ & FD Jena $_{2}$ & FD Jena $_{3}$ & FD Jena & Replicates per pool \\
\hline 1 & 8 & - & - & - & 8 \\
2 & 4 & 6 & 4 & 2 & 16 \\
3 & - & 4 & 4 & 4 & 12 \\
4 & - & 3 & 4 & 2 & 9 \\
8 & - & - & - & 1 & 1 \\
Replicates per pool & 12 & 13 & 12 & 9 & \\
\hline
\end{tabular}




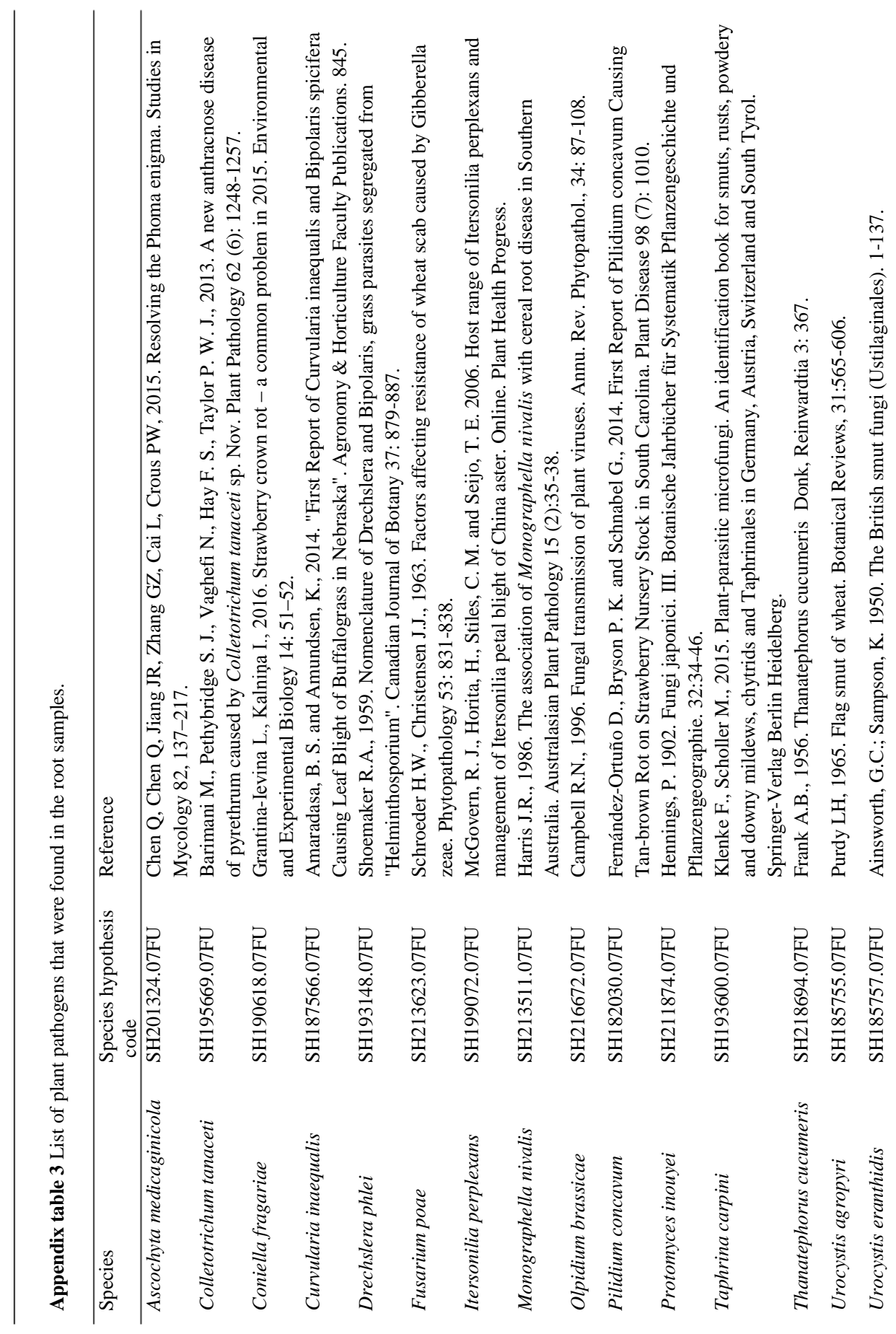


Root-associated fungal communities

The Jena Trait-Based Experiment. 
Chapter 4

\title{
Plant trait effects on richness and abundance in the soil food web
}

\author{
Sigrid Dassen \\ Gerlinde B. De Deyn \\ George A. Kowalchuk \\ Henk Duyts \\ S. Emilia Hannula \\ Basten Snoek \\ Nico Eisenhauer \\ Anne Ebeling \\ Cameron Wagg \\ Wim H. van der Putten
}




\section{Summary}

Multiple studies have shown that plant traits can partially explain the richness and abundance of soil organisms. However, little is known about how plant traits influence diversity and abundance of different taxa across the soil food web. In this study, we sought to examine how plant traits alter the richness and community composition of bacteria, fungi and nematodes from the green (living plant-based) and brown (dead plant-based) pathways of the soil food web. We analysed bacteria, fungi and nematodes in the Jena Trait Based Experiment in which grassland plant functional diversity is experimentally manipulated. We used next-generation sequencing and microscopy to identify and quantify fungal plant pathogens, root feeding nematodes, and arbuscular mycorrhizal fungi (AMF) representing the base of the green pathway, as well as bacterial and fungal saprophytes as the base of the brown pathway. We considered bacterial and fungal-feeding nematodes, omnivores, and predator nematodes as the higher trophic positions that feed on both the green and brown pathways in the soil food web. We tested the hypotheses that (1) plant species richness, plant functional diversity and trait identity have their strongest effects on richness and abundance of organisms in the green pathway, and that (2) effects of plant traits most strongly influence the lower trophic positions in the soil food web.

Plant species richness enhanced the total abundance of bacterial and fungal-feeding nematodes and reduced the relative abundance of fungal saprophytes. Plant functional diversity was positively related to bacterial richness, whereas plant species richness did not have an effect. However, plant functional diversity and species richness did not affect the richness or abundance of the other examined soil organisms. In contrast, plant trait identity affected the richness of AMF, plant pathogenic and saprotrophic fungi, as well of all feeding groups of nematodes except omnivores and predators. Moreover, trait identity effects were significant for the abundance of the organisms in the green pathway and for fungal feeders. Root length density had the greatest effect on fungal and nematode community composition; it was negatively related to the richness of AMF, fungal plant pathogens, fungal- and bacterial-feeding nematodes, and to the abundance of fungalfeeding nematodes. Still, the abundance of root feeding nematodes was positively related to root length density.

We conclude that plant trait composition affected the abundance of soil biota from the green pathway in the soil food web more than from the brown pathway, and that plant trait 


\section{Chapter 4}

composition also influenced the richness of fungi and nematodes. Furthermore, differences in plant trait composition have the strongest impact on the abundance of organisms occupying the lowest trophic positions in the soil food web. 


\section{Introduction}

An increasing number of studies has reported that plant species richness positively affects richness and community composition of soil biota. Increases in plant species richness and plant functional group diversity may relate positively to soil microbial or nematode richness (Sugiyama et al. 2008; Eisenhauer et al. 2011; Cortois et al. 2017; Dassen et al. 2017). Increasing microbial richness under enhanced plant species richness is probably due to specific plant-microbe specialisations, i.e. mycorrhizal associations or specific litter decomposers, whereas nematode richness may be caused by increasing variety in food resource quality (De Deyn et al. 2004; Cortois et al. 2017). Enhanced functional diversity can increase opportunities for microbial and nematode richness. However, there is far less evidence related to the impact of plant traits on belowground biodiversity as compared to studies targeting the effects of plant species diversity. Here, we separate effects of plant species richness from those of plant traits on the community composition of soil organisms.

Plant traits are often found to be related to the richness and abundance of soil biota. For example, plants with high nitrogen content have a positive effect on plant feeding nematode abundance (Cortois et al. 2017), bacterial abundance (Orwin et al. 2010; de Vries et al. 2012) and alter bacterial community composition (Moreau et al. 2015; Sayer et al. 2017). High plant nitrogen content is often associated with fast-growing plants, which have generally high specific leaf area (Reich 2014). Apart from plant chemical traits, structural traits also can be important for belowground organisms that directly or indirectly feed on living or dead plants. For example, mycorrhizal abundance and species richness are related to specific root length and to root length density (Cortois et al. 2016). To date, trait-based studies in relation to soil biota have mainly focused on plant chemical traits, and we therefore, focus on plant structural traits in the current study.

Given the complexity of the soil food web, it can be useful to group organisms by the pathway of their food origin or their trophic positions within the soil food web. Soil organisms that feed on living plants or live in symbiosis with plants have been characterized as being part of the so-called green pathway of the soil food web, while organisms that feed on dead plant residues are considered part of the brown pathway (Moore et al. 2004; Krumins et al. 2013). One might expect organisms within the green pathway to be more affected by plant traits than organisms in the brown pathway because they are more directly exposed to the plant traits, whereas organisms from the brown 


\section{Chapter 4}

pathway may feed on detritus that has already been pre-processed by other soil biota, for example bacteria feeding on plant residues that have been fragmented by earthworms.

Microbes and lower trophic positions of nematodes may be more sensitive to changes in plant community composition than higher trophic position nematodes (De Deyn et al. 2004; Berg \& Smalla 2009; Cortois et al. 2017; Glavatska et al. 2017). In a full aboveground and belowground assessment of the Jena plant diversity experiment, plant diversity effects diminished with increasing trophic position in the food web and plant diversity effects percolated more strongly aboveground than belowground (Scherber et al. 2010). It was assumed that the difference between above and belowground higher trophic positions was caused by generalism among consumers (Scherber et al. 2010), which is more profound below than above ground (Bardgett \& Wardle 2010). Such responses to plant trait composition have not yet been examined.

In spite of the fact that multiple studies have shown that plant traits can partially explain the richness or abundance of specific functional groups of soil organisms (Bardgett et al. 2014; Legay et al. 2014; Leff et al. 2018), little is known about how plant traits influence the diversity and abundance of different taxa across the soil food web (Porazinska et al. 2003). However, analyzing the entire soil food web can be a daunting task (Morriën et al. 2017). We therefore analyzed the basal part by sequencing the bacterial and fungal community, and by identifying and counting root feeding nematodes, and performed our analysis across higher trophic positions by identifying and counting microbivores, omnivores, and predators.

The aim of the present study is to analyse how changes in plant species richness, plant functional diversity and plant trait identity affect trophic groups of soil bacteria, fungi, and nematodes. We tested the hypotheses that: 1) Plant functional diversity and plant species richness positively relates to microbial and nematode richness in the bulk soil. We expected this relation to be stronger for organisms in the green as opposed to the brown soil pathway and that effects are strongest at lowest trophic positions. 2) Richness and abundance of microbes and nematodes in bulk soil depend on specific plant traits, and these effects are strongest for the organisms in the green pathway and at lowest trophic positions.

To test these hypotheses, soil samples were collected from the Trait-Based Experiment in Jena, Germany (Ebeling et al. 2014). At this grassland site, plant species richness and functional diversity have been experimentally manipulated. Three different species pools of 
eight plant species were defined using their relative positions along axes of functional diversity in plant morphological and developmental traits (Ebeling et al. 2014).

\section{Materials and methods}

\section{Experimental field site and design of the Trait-Based Experiment}

In August 2014, soil samples were collected from the plant Trait-Based biodiversity Experiment in Jena (Germany), which was started in 2010 and re-sown in 2011 (Ebeling et al. 2014). The established plant communities have different levels of trait complementarity, with functional diversity (named: $\mathrm{FD}_{\text {Jena }}$ ) ranging from plant communities with similar plant traits $\left(\mathrm{FD}_{\mathrm{Jena}} 1\right)$ to plant communities with high trait complementarity $\left(\mathrm{FD}_{\text {Jena }} 4\right)$. The plant structural traits that have been used to design the experiment were maximal rooting depth $(\mathrm{m})$, root length density $\left(\mathrm{cm}\right.$ root $/ \mathrm{cm}^{3}$ soil), plant height $(\mathrm{cm})$ and leaf area $\left(\mathrm{cm}^{2}\right)$, the plant development traits were growth start (week of the year) and flowering start (cumulative growing degree days $\left({ }^{\circ} \mathrm{C}\right)$ ). From all 60 plant species in the Jena Main Experiment (Roscher et al. 2004) those six traits have been determined and analysed by principal component analysis, yet for the design of the Trait Based experiment the legume species were excluded. Along the first axis plant species separated according to plant structural traits, whereas along the second axis plant species separated according to plant development traits (Appendix Fig. 1). The plant species along the first and second axes are grouped into the so-called pools 1 and 2, respectively. The plant species in the four corners of the ordination diagram are grouped into plant pool 3. A few plant species have traits that overlap and are, therefore, present in two pools (Appendix Fig. 1).

Each pool was subdivided into 4 evenly spaced sectors, and within each sector two plant species were chosen to represent the sector of that particular pool (Appendix Table 1). The distance between the sectors determines the level of plant community functional diversity (FD Jena $_{\text {a }}$. For example, a plant community with plant species from the same sector had $\mathrm{FD}_{\mathrm{Jena}}=1$, and a plant community with plant species from two neighbouring sectors had $\mathrm{FD}_{\text {Jena }} 2$. There were 138 plant communities: monocultures, 2, 3, 4, or 8 species mixtures, the combinations of $\mathrm{FD}_{\text {Jena }}$ levels and plant species richness (Appendix Table 2). The experiment has 3 blocks. Each block is a replication of the functional diversity gradient and plant species richness gradient, whereby plant identities within each plant community at each level of plant species and functional diversity vary among the blocks in order to avoid 


\section{Chapter 4}

sampling effects. For further details on the design see Ebeling et al. (2014). The plots in the Jena Experiment were mown twice a year following the biomass harvest and were hand weeded three times a year to remove non-sown plant species.

\section{Soil sampling}

Soil samples of $2.5 \mathrm{~cm}$ in diameter and $15 \mathrm{~cm}$ deep were collected from all 138 plots, using a corer. Per plot 4 cores were collected along the sides of the central square metre in each plot. These 4 soil samples per plot were pooled and sieved using a $2 \mathrm{~mm}$ mesh to remove plant roots and other large organic material. From each sieved sample, a $2 \mathrm{~mL}$ Eppendorf was filled with soil and stored at $-20{ }^{\circ} \mathrm{C}$ until DNA extraction. The remainder of the sieved soil was kept at $4{ }^{\circ} \mathrm{C}$ to be used for nematode extraction.

\section{DNA isolation and processing of sequences}

DNA was extracted from $0.25 \mathrm{~g}$ soil with the PowerSoil ${ }^{\circledR}$ DNA Isolation Kit (MoBio, Carlsbad, CA, USA). The fungal ITS2 region was amplified with primer pair ITS4/ITS9 (Ihrmark 2012). Bacterial 16S rRNA gene fragments were amplified using the primer combination 515f/806r (Bates et al. 2011). Both ITS and 16S had 12 base pair multiplex identifier tags on the primers. Amplicons of 16S and ITS were purified with Agencourt AMPure XP (Beckman Coulter, Brea, US) protocol according to the manufactures protocol. Subsequently, purified amplicons were quantified with Standard Sensitivity NGS Fragment Analysis kit (Advanced Analytical, Ankeny, US). Equal molar amounts on each sample were pooled, and send to BGI (Hong Kong) for Illumina Miseq PE 250 sequencing.

ITS sequences were analysed using the PIPITS pipeline (Gweon et al. 2015). To summarise the process, raw sequences were paired and quality filtered with the FASTX-Toolkit, ITS2 regions were extracted with ITSx, singletons removed and then clustered into OTUs with VSEARCH at $97 \%$ sequence similarity, removal of chimeras was done with UNITE UCHIME and then taxonomy was assigned to the sequences with the RDP Classifier against the UNITE fungal ITS database. In FUNGuild (Nguyen et al. 2016) OTUs were classified to potential function. Those OTUs that could be classified were grouped into saprophytes, AMF, plant pathogens (at species level, checked with literature), endophytes, and rest (EM, fungal/animal/unidentified plant pathogens).

The 16S rRNA gene raw paired-end Miseq data was processed into an annotated OTU table using the following pipeline. The RDP extension to PANDASeq (Masella et al. 2012) 
Assembler (Cole et al. 2014) was used to merge the raw reads using a minimum overlap of $150 \mathrm{bp}$ and a minimum PHRED score of 25. Primer sequences were removed from the FASTQ files using Flexbar version 2.5 (Dodt et al. 2012). Sequences were converted to FASTA format and concatenated into one file. Sequence clustering was done using VSEARCH version 1.0.10 (Rognes et al. 2016) at $97 \%$ identity. The strategy used for this was de-replication, sorting by abundance (at least 2 sequences) and clustering using the UCLUST smallmem algorithm (Edgar 2010). Thereafter, chimeric sequences were detected and removed using the UCHIME algorithm (Edgar et al. 2011) implemented in VSEARCH. Lastly, taxonomic classification for each OTU was obtained by using the RDP Classifier version 2.10 using default settings (Cole et al. 2014). The pipeline was made with Snakemake (Köster \& Rahmann 2012) as available at DOI: https://doi.org/10.5281/zenodo.597131 (de Hollander 2016).

\section{Nematode extraction}

Nematodes were extracted from $100 \mathrm{~g}$ fresh soil using Oostenbrink elutriators, this technique separates floating nematodes from heavier soil particles (Oostenbrink 1960). The nematodes were collected on four stacked sieves, one $75 \mu \mathrm{m}$ sieve on top of three $45 \mu \mathrm{m}$ sieves. Nematodes were rinsed of the sieves with tap water onto a double layer of cotton filters that was placed in $100 \mathrm{~mL}$ tap water for 24 hours at room temperature. During this step, vital nematodes migrate through the filter into the water. The tap water with nematodes was collected in glass jars, when the nematodes were settled at the bottom,the total volume was reduced to $2 \mathrm{~mL}$. Subsequently, the nematodes were fixated by adding 4 $\mathrm{mL}$ hot and $4 \mathrm{~mL}$ cold formalin of $4 \%$ (v/v). Nematode total abundances of each sample were counted in $2 \mathrm{~mL}$, and 150 nematodes per sample were morphologically identified to family or genus level using an inverted microscope. Nematodes were assigned to feeding groups according to Yeates et al. 1993 (Appendix Table 3).

\section{Data analysis}

The effect of plant species richness, plant functional diversity, percentage grass cover, percentage forb cover, plant biomass, and plant pool on overall bacterial, fungal and nematode community composition was tested in three separate distance-based redundancy analyses (db-RDA) with forward selection of explanatory plant community variables. Bacterial, fungal and nematode relative abundance data were normalised by centred log 


\section{Chapter 4}

ratio transformation and community distances were calculated with Hellinger transformation. Block was added as co-variate, and explanatory variables were included into the model if $\mathrm{P}_{\text {adj }}<0.05$. Then, the community weighted means of the specific plant traits (root length density, rooting depth, leaf area, plant height, growth start, and flowering start) were calculated using the plant species-specific aboveground biomass. Subsequently, the effect of the community weighted mean plant traits on overall bacterial, fungal and nematode community composition was tested in three separate distance-based redundancy analyses (db-RDA) with forward selection of explanatory plant community variables. Again, block was added as co-variate, and explanatory variables were included into the model if $\mathrm{P}_{\mathrm{adj}}<0.05$.

For the analyses of plant (trait) diversity effects on food web pathways, organisms were grouped into: fungal plant pathogens, plant feeding nematodes, AMF (basis of the green pathway), all bacteria, fungal saprophytes (basis of the brown pathway), and bacterivores, fungal-feeding nematodes, omnivores, and predatory nematodes (to analyse diversity effects on the higher trophic positions in the soil food web).

Richness of bacteria, fungal and nematode groups were analysed using a linear mixed model with plant pool, (log) sown plant species richness, plant functional diversity, plant biomass, percentage grass cover, and percentage forb cover as main fixed factors. The model also included two-way interactions between plant pool and one of the other factors. Block was included as a random factor. Sown plant species richness was added into the model first and then plant functional diversity, because the levels of plant species richness and functional diversity were not fully factorial (Appendix Table 2). In this way the effect of plant functional diversity was tested after accounting for the effect of monocultures and eight plant species mixtures. The effect of community-weighted mean of the plant traits on the richness of bacteria, fungal and nematode groups were also analysed by linear mixed models. The plant traits were fixed factors, whereas block was used a random factor.

Relative abundance of fungal groups was transformed prior to analyses by linear mixed models. Relative abundance of fungal plant pathogens was log transformed, AMF was square root transformed and for fungal saprophytes square transformation was used to obtain a normal distribution. In the models, plant pool, (log) sown plant species richness, plant functional diversity, plant biomass, percentage grass cover, and percentage forb cover were main fixed factors. The model also included two-way interactions between plant pool and one of the other factors. Block was used as a random factor. The effect of community weighted mean of the plant traits on the relative abundance of fungal groups were also 
analysed by linear mixed models. The plant traits were fixed factors and block was a random factor.

Because there is little documentation on mixed models with negative binomial error distributions the total abundances of nematode groups were analysed using negative binomial generalized linear models with block, plant pool, (log) sown plant species richness, plant functional diversity, plant biomass, percentage grass cover, and percentage forb cover as main fixed factors. The model also included two-way interactions between plant pool and one of the other plant factors. The effect of community weighted mean of the plant traits on the total abundances of nematode groups were analysed by negative binomial generalized linear models too. Block and the plant traits were fixed factors and there were no interaction terms in the model.

The mixed models, negative binomial generalized linear models and post hoc analyses were performed in R with package nlme (Pinheiro et al. 2015), MASS (Venables \& Ripley 2002), phia (Helios De Rosario-Martinez 2015). The db-RDAs were made in CANOCO version 5.0. Figures were made in R with package 'ggplot' (Wickham 2009) or CANOCO and further edited in Adobe Illustrator.

\section{Results}

In total, 14,038 bacterial OTUs were identified from 2,519,433 high-quality reads, and 6601 fungal OTUs from 2,632,306 high-quality reads. Among the 6601 fungal OTUs, 546 could be identified as AMF OTUs, and 1030 as saprophytes. In total 25 potential plant pathogens could be identified at the species level and corroborated for pathogenicity based on literature. The number of nematode taxa per feeding group were 25 bacterial feeders, 7 fungal feeders, 23 plant feeders, 9 omnivores, and 9 predators.

\section{Bacterial, fungal and nematode community composition}

There was no significant effect of plant species richness and plant functional diversity on overall bacterial, fungal and nematode community composition as determined by distancebased redundancy analysis with forward selection of factors. Furthermore, none of the other factors (plant traits, grass and forb cover, plant biomass or plant pool) explained a significant portion of the variation in bacterial community composition. In contrast, fungal community composition was significantly impacted by specific plant traits, with root length 


\section{Chapter 4}

density, leaf area, plant height, growth start, and flowering start explaining $6.1 \%$ of the total variation $\left(\mathrm{P}_{\mathrm{adj}}<0.05\right)$, grass cover explaining 2.2\% (pseudo-F $\left.=2.6, \mathrm{P}_{\mathrm{adj}}=0.02\right)$, and forb cover explaining $1.0 \%$ of the total variation (pseudo-F $=1.2, \mathrm{P}_{\mathrm{adj}}=0.03$ ) (Fig. 1). Community composition of nematodes was also significantly affected by specific plant traits, notably by root length density (explained variation $1.5 \%$, pseudo- $\mathrm{F}=2.1, \mathrm{P}_{\mathrm{adj}}=0.01$ ) and grass cover (explained variation $1.5 \%$, pseudo-F $=2.0, \mathrm{P}_{\mathrm{adj}}=0.03$ ) (Fig. 1). Plant pool explained $1.1 \%$ of the variation observed in fungal community composition (pseudo- $\mathrm{F}=$ $1.4, \mathrm{P}_{\mathrm{adj}}=0.02$ ) and $2.3 \%$ for nematode community composition (pseudo-F $=3.2, \mathrm{P}_{\mathrm{adj}}=$ $0.02)$.

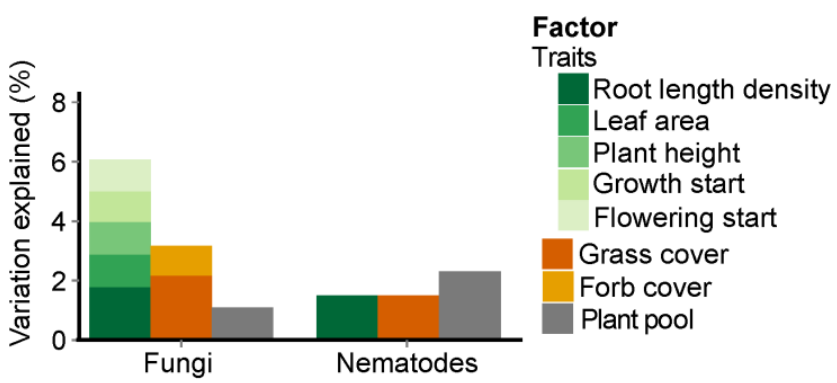

Figure 1 Overview of variation explained in overall fungal and nematode community composition by plant traits, grass and forb percentage cover and plant pool from the db-RDA analyses. The figure sums up the factors that significantly contributed to overall fungal and nematode community composition.

\section{Bacterial, fungal and nematode richness}

\section{Green pathway in the soil food web}

There was no significant main effect of plant species richness or functional diversity on the richness of organisms at the base of the green pathway in the soil food web ( $\mathrm{P} \geq 0.1$ for all groups). However, there was an interaction effect of plant species richness and plant pool on AMF richness $\left(\mathrm{F}_{2,102}=3.73, \mathrm{P}=0.03\right)$. For plant species in pool 2, AMF richness was positively related to plant species richness $\left(\mathrm{F}_{1,34}=6.08, \mathrm{P}=0.02\right)$, but this was not the case in pools 1 and $3\left(\mathrm{~F}_{1,34}=1.28, \mathrm{P}=0.27\right.$ and $\mathrm{F}_{1,30}=3.66, \mathrm{P}=0.07$, respectively). All plant traits, except rooting depth and growth start, were related to richness of organisms at the base of the green pathway (Fig. 2). Root length density was negatively related to AMF and plant pathogen richness $\left(\mathrm{F}_{1,115}=7.86, \mathrm{P}=0.006\right.$ and $\mathrm{F}_{1,115}=7.25, \mathrm{P}=0.008$, respectively $)$, whereas plant height and flowering start were positively related to AMF and plant pathogen richness $\left(\mathrm{AMF}: \mathrm{F}_{1,115}=5.82, \mathrm{P}=0.02\right.$ and $\mathrm{F}_{1,115}=13.22, \mathrm{P}=0.0004$ and plant pathogens: $\mathrm{F}_{1,115}=6.67, \mathrm{P}=0.01$ and $\left.\mathrm{F}_{1,115}=7.17, \mathrm{P}=0.009\right)$. Leaf area was negatively related to $\mathrm{AMF}$ and plant feeder richness $\left(\mathrm{F}_{1,115}=4.43, \mathrm{P}=0.04\right.$ and $\mathrm{F}_{1,128}=7.10, \mathrm{P}=0.009$, 
respectively). Percentage grass cover had a negative effect on $A M F$ richness $\left(F_{1,102}=8.26\right.$, $\mathrm{P}=0.005)$, but a positive effect on plant pathogen richness $\left(\mathrm{F}_{1,102}=5.19, \mathrm{P}=0.02\right)$. There were no main effects of forb cover, plant biomass and plant pool on richness of organisms at the base of the green pathway.

\section{Brown pathway in the soil food web}

Bacterial and fungal saprophyte OTU richnesses were not significantly affected by plant species richness $\left(\mathrm{F}_{1,100}=0.66, \mathrm{P}=0.42\right.$ and $\left.\mathrm{F}_{1,102}=0.26, \mathrm{P}=0.61\right)$. However, bacterial OTU richness was positively related to functional diversity $\left(\mathrm{F}_{1,100}=5.09, \mathrm{P}=0.03\right)$, whereas fungal saprophyte OTU richness was not $\left(\mathrm{F}_{1,102}=0.17, \mathrm{P}=0.68\right)$. None of the six plant traits examined had a significant effect on bacterial OTU richness $(\mathrm{P} \geq 0.07$ for all traits) (Fig. 2). In contrast, fungal saprophyte richness increased with plant height and flowering start $\left(\mathrm{F}_{1,115}=6.39, \mathrm{P}=0.01\right.$ and $\mathrm{F}_{1,115}=8.31, \mathrm{P}=0.005$ resp. $)$. There were no main effects of grass or forb cover, plant biomass and plant pool on richness of organisms at the base of the brown food web.

\section{Higher trophic positions of the green and brown pathways}

Plant species richness and functional diversity did not significantly affect richness of higher trophic positions in the soil food web. However, specific plant traits showed a range of significant effects. The richness of both fungal and bacterial feeders were negatively related to root length density $\left(F_{1,128}=6.72, P=0.01\right.$ and $F_{1,128}=8.66, P=0.004$, respectively $)$. In addition, the richness of fungal feeders and predators was negatively related to leaf area $\left(\mathrm{F}_{1}\right.$, ${ }_{128}=5.42, \mathrm{P}=0.02$ and $\mathrm{F}_{1,128}=4.18, \mathrm{P}=0.04$ resp.), and fungal feeder richness was negatively related to plant biomass $\left(\mathrm{F}_{1,117}=4.16, \mathrm{P}=0.04\right)$. Positive relations were also found, as fungal and bacterial feeder richness were positively related to percentage cover of forbs $\left(F_{1,117}=4.32, P=0.04\right.$ and $F_{1,117}=4.92, P=0.03$ resp. $)$. Predator richness differed between the plant pools $\left(\mathrm{F}_{2,117}=5.15, \mathrm{P}=0.007\right)$. Predator richness was lower in pool 3 than in pool $2\left(\chi_{1}^{2}=10.73, \mathrm{P}=0.003\right)$, with no significant differences detected when comparing pool 1 with pool 2 or pool 1 with pool 3 . 


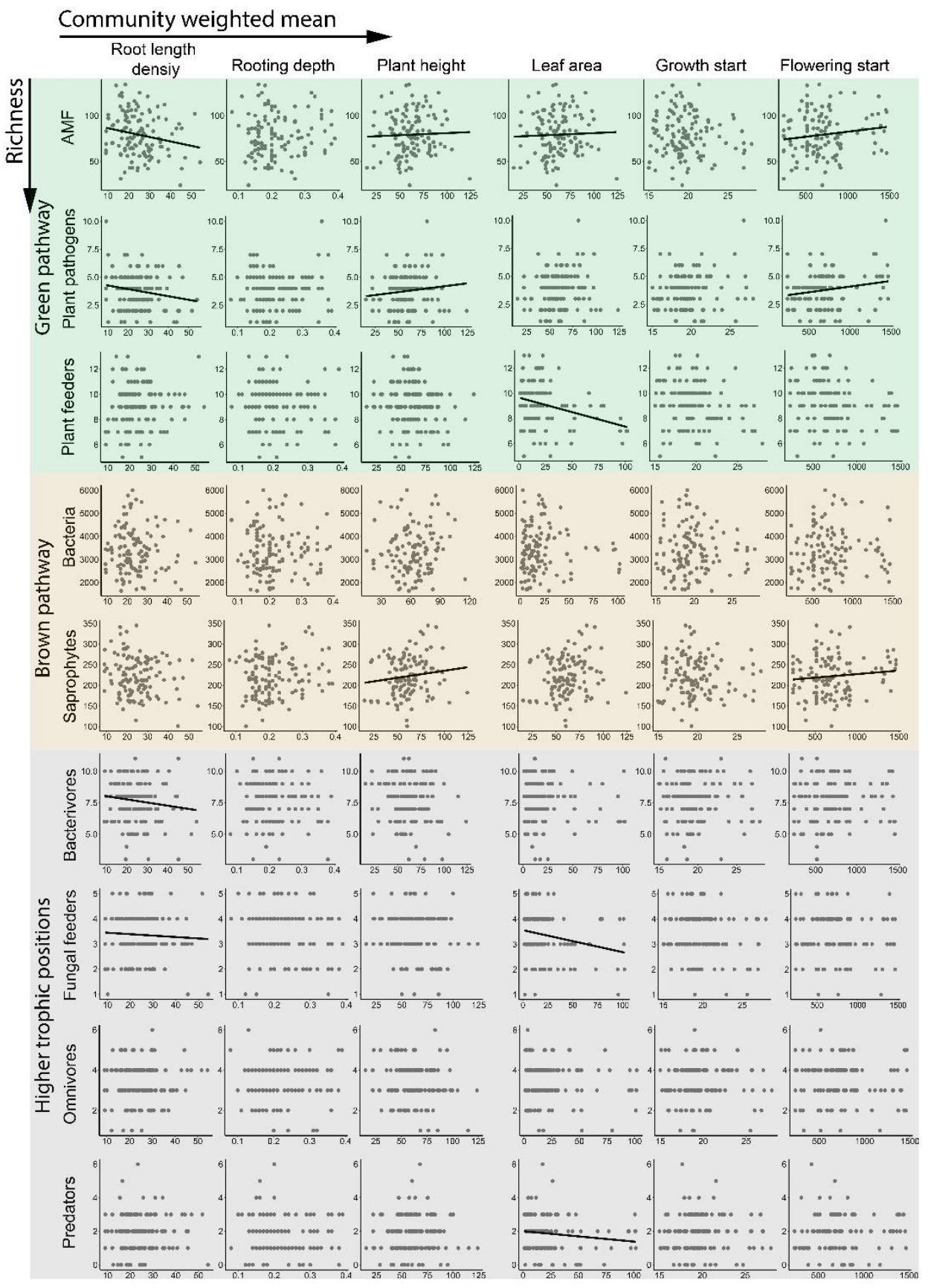

Figure 2 Plant trait effects on richness of soil biota from the base of the green and brown pathways of the soil food web and of higher trophic positions. The traits are the community weighted mean of: maximal rooting depth (m), root length density $\left(\mathrm{cm}\right.$ root $/ \mathrm{cm}^{3}$ soil), plant height $(\mathrm{cm})$ and leaf area $\left(\mathrm{cm}^{2}\right)$, growth start (week of the year) and flowering start (cumulative growing degree days $\left({ }^{\circ} \mathrm{C}\right)$ ). Trend lines indicate significant interactions $(\mathrm{P} \leq 0.05)$. 


\section{Fungal and nematode abundance}

\section{Green pathway of the soil food web}

There were no significant main effects of plant species richness and functional diversity on the relative or total abundance of species at the base of the green food web. However, there was a trend that plant pathogen relative abundance was negatively related to plant species richness $\left(\mathrm{F}_{1,102}=3.63, \mathrm{P}=0.06\right)$. Plant functional diversity affected AMF relative abundance, but this effect depended on the plant pool (functional diversity x plant pool $\mathrm{F}_{2}$, $102=3.13, \mathrm{P}=0.048)$. As this effect was already marginal, post-hoc analysis no longer showed significant differences between plant pools. Overall, the abundance of each group of soil biota at the base of the green pathway was affected by plant traits, yet the trait that exerted the strongest effect differed among the groups of soil biota examined. For example, the relative abundance of $A M F$ was negatively related to leaf area $\left(F_{1,115}=7.30, P=0.008\right)$, plant pathogen relative abundance was positively related to growth start $\left(\mathrm{F}_{1,115}=10.68, \mathrm{P}=\right.$ $0.001)$, and plant feeder total abundance was positively related to root length density $\left(\mathrm{F}_{1,128}\right.$ $=11.55, \mathrm{P}=0.0009)$ and to grass cover $\left(\mathrm{F}_{1,128}=12.80, \mathrm{P}=0.0003\right)$, and negatively to flowering start $\left(\mathrm{F}_{1,128}=7.50, \mathrm{P}=0.007\right)$ (Fig. 3). Forb cover and plant biomass did not significantly influence the abundance of species at the base of the green pathway. The total abundance of plant feeders differed for the specific plant pools $\left(\mathrm{F}_{2,132}=8.91, \mathrm{P}=0.0001\right)$. Plant feeder total abundance was lower in pool 3 than in pool $2\left(\chi_{1,117}^{2}=7.09, \mathrm{P}=0.02\right)$, with no significant differences detected when comparing pool 1 with pool 3 or pool 1 with pool 2. The relative abundance of plant pathogens was also different between the plant pools $\left(\mathrm{F}_{2,102}=3.90, \mathrm{P}=0.02\right)$. Plant pathogen relative abundance was lower in pool 1 than in pool $2\left(\chi_{1}^{2}=7.58, \mathrm{P}=0.02\right)$, with no significant differences detected when comparing pool 1 with pool 2 or pool 2 with pool 3 .

\section{Brown pathway of the soil food web}

The relative abundance of saprophytes decreased with increasing plant species richness $\left(\mathrm{F}_{1}\right.$, $102=6.07, \mathrm{P}=0.02)$. In contrast, plant functional diversity, specific plant traits, percent cover of grasses or forbs and plant biomass all had no significant effect on the relative abundance of saprophytes ( $\mathrm{P} \geq 0.1$ for all factors). The relative abundance of saprophytes differed between the plant pools $\left(\mathrm{F}_{1,102}=3.99, \mathrm{P}=0.02\right)$. Fungal saprophyte relative 


\section{Chapter 4}

abundance was higher in pool 3 than in pool $2\left(\chi_{1}^{2}=7.83, \mathrm{P}=0.02\right)$, with no significant differences detected when comparing pool 1 with pool 3 or pool 1 with pool 2.

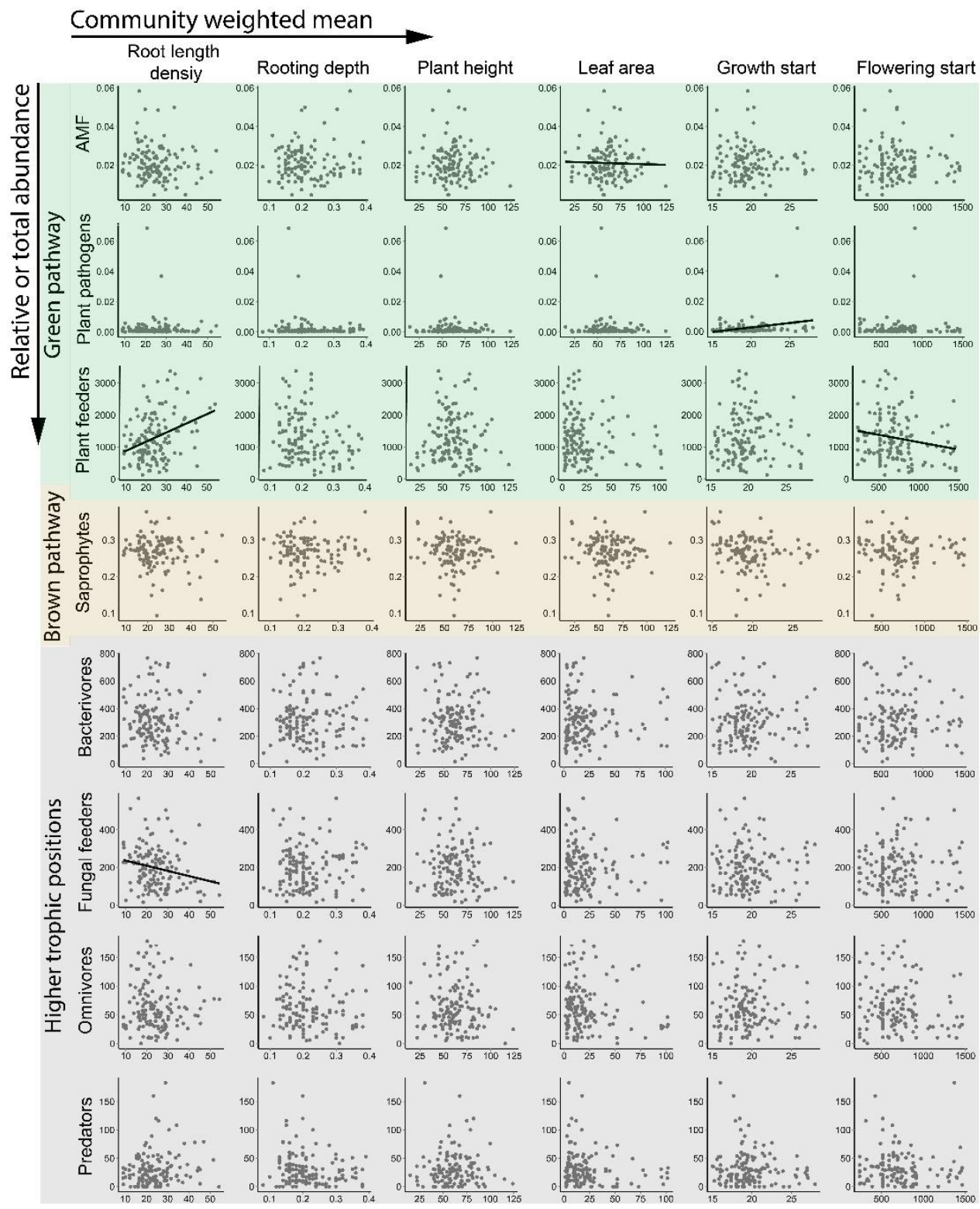

Figure 3 Plant trait effects on relative abundance (AMF, fungal plant pathogens fungal saprophytes) and total abundance (nematode feeding groups) from the base of the green and brown pathway of the soil food web and of higher trophic positions. The traits are the community weighted mean of: maximal rooting depth (m), root length density $\left(\mathrm{cm}\right.$ root $/ \mathrm{cm}^{3}$ soil), plant height $(\mathrm{cm})$ and leaf area $\left(\mathrm{cm}^{2}\right)$, growth start (week of the year) and flowering start (cumulative growing degree days $\left({ }^{\circ} \mathrm{C}\right)$ ). Trend lines indicate significant interactions $(\mathrm{P} \leq 0.05)$. 


\section{Higher trophic positions of the green and brown pathways}

The total abundance of fungal and bacterial feeders increased with plant species richness $\left(F_{1,131}=5.31, P=0.02\right.$ and $F_{1,131}=5.39, P=0.02$ resp. $)$. However, the total abundance of predators and omnivores was not affected by plant species richness ( $\mathrm{P} \geq 0.1$ for all groups). Likewise, none of the higher trophic positions showed total abundances that were significantly impacted by plant functional diversity ( $\mathrm{P} \geq 0.1$ for all groups). Specific plant traits exerted some effects. Notably, the total abundance of the fungal-feeding nematodes was negatively related to root length density $\left(\mathrm{F}_{1,128}=10.37, \mathrm{P}=0.002\right)$ (Fig. 3). In addition, fungal feeder total abundance was also negatively related to the percentage cover of both grasses and forbs $\left(\mathrm{F}_{1,128}=4.85, \mathrm{P}=0.03\right.$ and $\mathrm{F}_{1,127}=4.09, \mathrm{P}=0.04$ resp. $)$. The total abundance of bacterial feeding nematodes was positively related to forb cover $\left(F_{1,127}=\right.$ $6.29, \mathrm{P}=0.01)$. Abundances of predators and omnivores were not significantly related to percentage cover of grass or forbs, and plant biomass did not affect the abundances of at the higher trophic positions. Predator total abundance was significantly different between the plant pools $\left(\mathrm{F}_{1,132}=3.24, \mathrm{P}=0.04\right)$, however, after post-hoc testing, no significant differences were detected, which is reasonable given the fact that post hoc tests are generally slightly more conservative than the overall test.

\section{Discussion}

We assessed the effects of plant species richness, functional diversity and specific plant traits on the richness and abundance of the various groups of organisms in the soil food web. We distinguished between lower trophic positions in the green and brown pathways, as well as higher trophic position organisms that may feed on both pathways. We tested two major hypotheses: (1) that plant species richness, plant functional diversity and trait identity have their strongest effects on the richness and abundance of organisms in the green pathway, (2) that lower positions within the soil food web are more affected that higher trophic positions. Partially in support of the first hypothesis, we found that the richness and abundance of all groups in the green pathway were affected by specific plant traits, but not by plant species richness or functional diversity. However, organisms in the brown pathway were also affected: specific plant traits influenced fungal saprophytes richness, plant functional diversity affected bacterial richness, and fungal saprophyte abundance was influenced by plant species richness. Partially in support of our second hypothesis, we 


\section{Chapter 4}

found that specific plant traits altered the abundance of soil biota at lower trophic positions more than at higher tropic positions. However, specific plant traits altered the richness of soil biota at both lower and higher trophic positions.

\section{Green and brown pathways}

We examined whether plant community composition differently affected the richness and abundance of biota in the green and brown pathways. Our results show that species richness and abundance of the green pathway is influenced by specific plant traits, but not by plant species richness and functional diversity. In the brown pathway, richness and abundance was influenced by plant diversity effects, however, not so much by specific plant traits. Thus, specific plant traits and plant diversity differentially influence the richness and abundance of groups within the brown versus green pathways. This differential influence might be related to effects of plant community composition on resource quality and quantity as perceived by the different soil biota. For example, organisms in the brown pathway of the food web feed on plant residues that range from low to high $\mathrm{C}: \mathrm{N}$ ratios and depending on this ratio grow faster or slower (Krumins et al. 2013). Not only is the C:N ratio of importance, but also the physical structure or organic material play a role. It is important whether the resources are presented in the form of plant tissue or in the form of less complex and smaller molecules in the rhizosphere, as is the case for root exudates. Plant species richness is positively related to rooting density (Oram et al. 2018), which may enhance the amount of root exudates that become available for the soil food web (Hannula et al. 2012) and thereby increase the pool of high quality residues for the brown pathway. In contrast, organisms in the green pathway of the food web are in direct contact with plant roots, which in general have a high $\mathrm{C}: \mathrm{N}$ ratio (Krumins et al. 2013) and also experience specific plant defense responses to which they need to adapted. Increasing plant species richness can further increase C:N ratios of roots (Chen, Mommer et al. 2017) and shoots (Guiz et al. 2018), which could lead to further specialisation in the green pathway. Despite this possibility, we did not observe a relation between plant species richness and species richness in the green pathway of the soil food web. Instead, we found richness in the green pathway to be related to changes in specific plant traits, which might also be related to C:N ratios (Roscher et al. 2004; Guiz et al. 2018).

\section{Trophic positions}


We also examined whether changes in plant community composition affect the richness and abundance of soil biota in lower versus higher tropic positions differentially. Interestingly, changes in specific plant traits and percentage cover of the functional groups of plants affected soil biota at both lower and higher trophic positions in the soil food web. This finding is contrary to our second hypothesis, namely that changes in plant community composition influence the lower tropic positions more that the higher tropic positions. Our results indicate that plants affect richness of higher tropic positions indirectly, for example via changes in community composition of the lower tropic positions, or by causing abiotic or physical changes in soil.

Changes in plant community composition altered the abundance of soil biota at all lower tropic positions, whereas the abundance of microbial feeders was the only higher tropic group of soil biota to be influenced. Similar to the effects on richness, specific plant traits affected more groups of soil biota than plant functional group or plant species richness. These findings are in line with De Deyn et al. (2004) who found that plant identity had stronger effects on the abundance of nematode feeding groups than plant species richness, and that plant species identity affected the abundance of soil biota at lower tropic positions more than higher tropic positions. In contrast, previous observations from the Jena Main Experiment showed that plant species richness increased the abundance of all nematode feeding groups, except predators (Cortois et al. 2017). These somewhat contrasting findings may be due to the different time periods since the establishment of the experimental vegetation schemes. The samples examined by Cortois et. al. (2017) are nematode communities in the Main Experiment sampled 8 years after establishment, whereas our samples were taken in the Trait-Based Experiment 3 years after establishment. Plant community effects might have a delayed impact on higher tropic positions in the soil food web (Korthals et al. 2001), so that after 3 years they might not yet have emerged.

\section{Plant diversity versus specific traits}

We found rather limited effects of plant species richness and functional diversity on the richness and abundance of soil biota. Instead, plant traits were much more effective in influencing soil biota. Possibly, reduction in plant species richness or functional diversity did not remove particular traits from the plant community but rather reduced the variation of particular traits. Therefore, plant species richness and functional diversity generally have little effect on the richness and abundance of soil biota. Biota with higher host specificity, 


\section{Chapter 4}

e.g. biota in the green pathway, might be more sensitive to plant species loss. However, as opposed to the biota in the green pathway, we found that the richness and abundance of biota in the brown pathway and the abundance of microbial-feeding nematodes were affected by plant species richness or functional diversity. The effect of plant species richness and functional diversity on biota in the brown pathway and microbial-feeding nematodes might therefore be more related to increasing food sources, as discussed above.

Specific plant traits could explain more variation in fungal community composition than plant functional group, functional diversity or plant species richness. The community weighted means of the six plant traits were calculated using the proportions of plant species in the aboveground plant biomass. However, the trait values per plant species were determined in monocultures, whereas in the field plots, traits may have varied in response to neighbouring plants (Valverde-Barrantes et al. 2013; Lipowsky et al. 2015) and nutrient availability (Hodge 2004). This might have influenced the actual community weighted means of plant traits in the plant communities in the field. Nevertheless, we found that all specific plant traits, except rooting depth, were related to richness or abundance of at least one group of soil biota. If not confounded by trait plasticity, our findings indicate that the community weighted mean can explain variation in soil biota community composition at least to some extent. However, soil biota composition, especially in the green pathway, might be better explained by specific plant traits when root biomass instead of aboveground biomass is used in the analyses.

We showed that morphological and developmental plant traits may influence fungal and nematode richness and abundance. In contrast, other trait-based studies have focused mainly on chemical traits (Moreau et al. 2015; Cortois et al. 2017; Sayer et al. 2017) or a limited number of morphological traits (often specific leaf area and specific root length) together with chemical traits (de Vries et al. 2012; Legay et al. 2014; Cantarel \& Pommier 2015; Leff et al. 2018). Morphological and developmental traits as used in our study may reflect plant chemical properties, but they may also be related to characteristics that are linked to plant functions. For example, plants with low root length density or specific root length generally have more mycorrhizal colonisation (Cortois et al. 2016), because the hyphae fulfil the function of dense fine roots (Smith \& Read 2010). Morphological plant traits might also influence soil biota indirectly via changes in soil structure (Gyssels et al. 2005; Erktan et al. 2016). It is suggested that via mediation of soil biota, morphological as well as chemical plant traits play an important role in soil and ecosystem services (Díaz \& Cabido 2001; Faucon et al. 2017). 


\section{Conclusions}

We conclude that differences in plant trait composition affect the abundance of soil biota from the green soil food web more than soil biota from the brown soil food web. Plant trait composition, more than plant species richness and functional diversity, influenced richness and community composition of fungi and nematodes. Furthermore, differences in plant trait composition have a stronger effect on the abundance of lower trophic position organisms as compared to higher trophic position organisms. 


\section{Chapter 4}

\section{Supporting information}

Appendix table 1 Selected plant species per plant pool per sector, see also appendix figure 1.

\begin{tabular}{lllll}
\hline \multirow{2}{*}{ Pool } & Sector 1 & Sector 2 & Sector 3 & Sector 4 \\
\hline \multirow{2}{*}{1} & Festuca rubra & Avenula pubescens & Leucanthemum vulgare & Centaurea jacea \\
& Poa pratensis & Phleum pratense & Plantago lanceolata & Knautia arvensis \\
\multirow{2}{*}{2} & Holcus lanatus & Phleum pratense & Dactylis glomerata & Anthoxanthum odoratum \\
& Geranium pratense & Plantago lanceolata & Leucanthemum vulgare & Ranunculus arcis \\
& Prunella vulgaris & Cirsium oleraceum & Anthriscus sylvestris & Anthoxanthum odoratum \\
& Veronica chamaedrys & Sanguisorba officinalis & Rumex acetosa & Glechoma hederacea \\
\hline
\end{tabular}

Appendix table 2 Overview of the number of replicates of each plant species richness and functional diversity (FD Jena) level.

\begin{tabular}{llllll}
\hline Plant species richness & FD $_{\text {Jena }} 1$ & FD $_{\text {Jena }} 2$ & FD Jena $_{3}$ & FD $_{\text {Jena }} 4$ & Replicates per pool \\
\hline 1 & 8 & - & - & - & 8 \\
2 & 4 & 6 & 4 & 2 & 16 \\
3 & - & 4 & 4 & 4 & 12 \\
4 & - & 3 & 4 & 2 & 9 \\
8 & - & - & - & 1 & 1 \\
Replicates per pool & 12 & 13 & 12 & 9 & \\
\hline
\end{tabular}


Appendix table 3 Observed nematode taxa and their assignment to feeding groups according to Yeates et al. 1993.

\begin{tabular}{|c|c|c|c|c|}
\hline Bacterivores & Fungal feeders & Plant feeders & Omnivores & Predators \\
\hline Achromadoridae & Aphelenchidae & Anquinidae & Campydoridae & Aporcelaimidae \\
\hline Alaimidae & Aphelenchodidae & Belondiridae & Mononchidae & Discolaimidae \\
\hline Bastianiidae & Diphtherophoridae & Criconematidae & Qodsianematidae & Nygolaimidae \\
\hline Bunonematidae & Leptonchidae & Dolichodoridae & Thornenematidae & Tripylidae \\
\hline Chephalobidae & & Hoplolaiminae & & \\
\hline Cryptonchidae & & Longidoridae & & \\
\hline Diplopeltidae & & Meloidogynidae & & \\
\hline Microlaimidae & & Nordiidae & & \\
\hline Monhysteridae & & Paratilenchidae & & \\
\hline Neodiplogasteridae & & Pratylenchidae & & \\
\hline Panagrolaimidae & & Psilenchidae & & \\
\hline Plectidae & & Trichodoridae & & \\
\hline Prismatolaimidae & & Tylenchidae & & \\
\hline \multicolumn{5}{|l|}{ Rhabditidae } \\
\hline Wilsonematidae & & & & \\
\hline
\end{tabular}




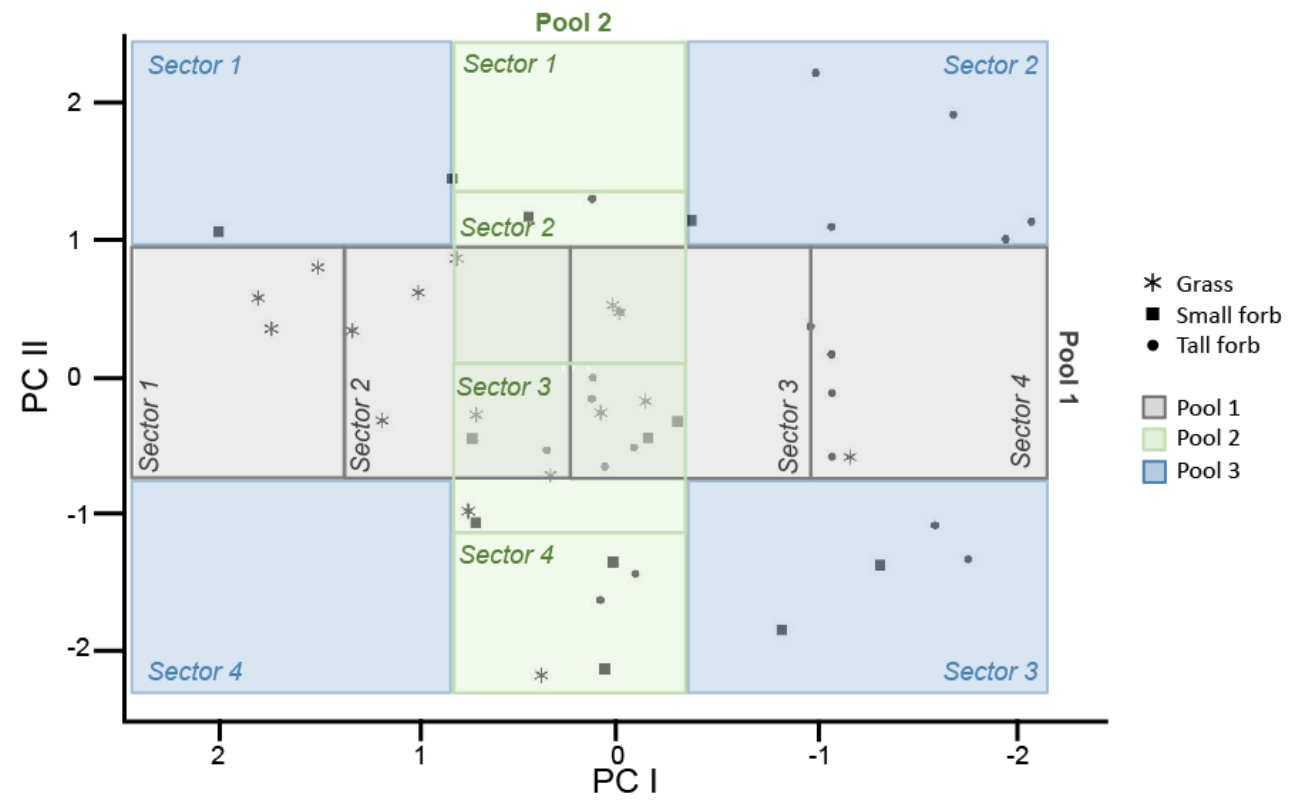

Appendix Fig. 1 Principal component analysis of six plant traits (maximal rooting depth, root length density, plant height and leaf area, growth start, and flowering start). Plant structural traits are maximised on the x-axis (Pool 1) and plant development traits are maximised on the y-axis (Pool 2). Within each sector two plant species are representative for the sector of the particular pool. The distance between the sectors determines the level of plant community functional diversity ( $\mathrm{FD}_{\text {Jena }}$ ). The figure is modified after Ebeling et al. (2014) and Steinauer et al. (2017). 


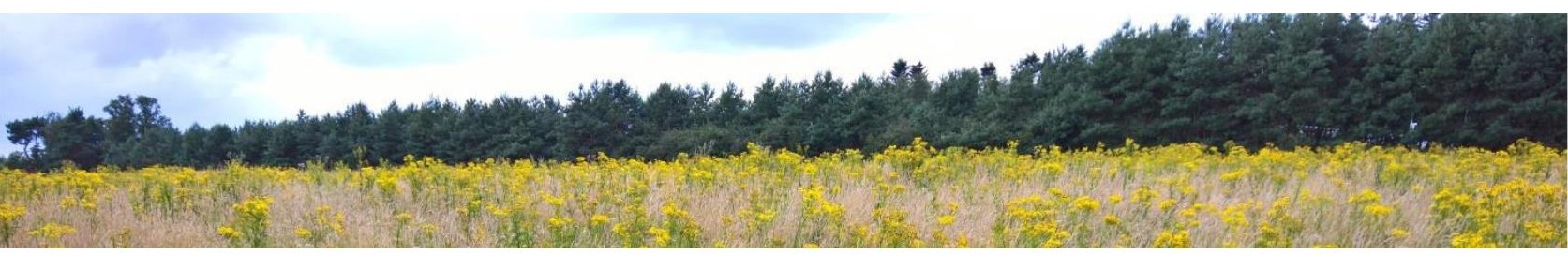

Ex-arable field at the Veluwe. 
Chapter 5

\section{Mycorrhizal networks in restoration grasslands suppress seedling biomass}

Sigrid Dassen

Wim H. van der Putten

Gerlinde B. De Deyn 


\section{Summary}

Establishment and growth of grassland species is generally promoted by arbuscular mycorrhizal fungi (AMF) when grown in isolation. However, in grassland communities AMF form networks that connect individuals within and between species. We examined how established mycorrhizal networks influence seedling performance using a 28 year chronosequence of ex-arable fields. During this succession, plant-soil feedback changes from negative in early to positive in mid-later stages.

We selected 4 grass and 4 forb species with negative or neutral-positive plant-soil feedback and grew them individually in mesh cores with live field soil. Cores were placed in 6 grasslands along the chronosequence. Half of all cores were regularly disturbed to disrupt in-growing mycorrhizal hyphae, which enabled examining mycorrhizal network effects on seedling growth.

Interruption of the ingrowing mycorrhizal network increased seedling biomass irrespective of succession stage, grass/forb group, or plant-soil feedback type. In seedlings in undisturbed cores, AMF colonisation was negatively correlated to seedling biomass.

We conclude that in secondary succession grasslands mycorrhizal networks reduced biomass of grass and forb seedlings, independent of negative or positive plant-soil feedback interactions. The level of biomass reduction in seedlings connected to a mycorrhizal network is associated with increased levels of AMF colonisation. 


\section{Chapter 5}

\section{Introduction}

Feedback interactions between plants and the soil microbial community are considered a key factor determining the establishment success of new plant generations (Bradford et al. 2002; Kardol et al. 2006b; Bennett et al. 2017). This plant-soil feedback (PSF) effect can be negative, neutral or positive, depending on the net effect of antagonistic and mutualistic soil biota on the focal plant species (Bever et al. 1997). Plant species that experience a negative PSF perform worse in soil conditioned by conspecifics than in a control soil (Van der Putten et al. 2013). Arbuscular mycorrhizal fungi (AMF) are thought to contribute to positive PSF, because they can increase plant nutrient supply (Aerts 2002; Read \& PerezMoreno 2003), stress resistance to drought (Allen \& Boosalis 1983), and reduce susceptibility to root pathogens (Harrier \& Watson 2004; Wehner et al. 2011). Although experiments with individual plants generally show that AMF colonisation is beneficial for plant establishment and growth, experiments with seedlings in plant communities demonstrate that the presence of AMF does not necessarily lead to increasing seedling survival (Bennett et al. 2017) or seedling biomass (Moora \& Zobel 1996). These differential outcomes indicate that our understanding of whether AMF facilitate or suppress seedling establishment and growth in natural systems, and how this may depend on the net PSF effect that plant species develop, needs to be further elucidated.

Presence of AMF in soil does not necessarily lead to a net positive soil effect, because in living soil with a natural diversity of soil biota, including many non-AMF microorganisms, antagonists may overrule the positive effects of AMF (Mora-Romero et al. 2015; Bennett et al. 2017). Moreover, not all AMF species are equally beneficial and some may even suppress plant growth depending on host plant identity (Johnson et al. 1997; Klironomos 2003), host plant diversity (Hart et al. 2003), and soil $\mathrm{N}$ and $\mathrm{P}$ availability (Hoeksema et al. 2010). Another situation in which AMF can negatively influence seedling establishment and growth is in naturally mixed plant communities, where seedlings are exposed to an established community of mycorrhizal fungi, their host plants, and to their associated microbes (Lekberg \& Koide 2005; Buée, Boer et al. 2009). AMF can form hyphal networks that connect multiple plants of the same (conspecific), as well as different (heterospecific) species (Francis \& Read 1995; Van der Heijden \& Horton 2009; Simard et al. 2012). Nutrients (Meding \& Zasoski 2008; Mikkelsen et al. 2008) and signalling compounds (Achatz \& Rillig 2014; Johnson \& Gilbert 2015) can then be transferred via these mycorrhizal networks. Multiple studies have shown that mycorrhizal fungi do not equally distribute, or trade, their goods among plants within the network, so that some plants might 
benefit more from association with the network than others. The supply of nutrients via mycorrhizal networks to plants depends on host quality (carbon supply to the AMF partner) (Fellbaum et al. 2014), sink strength (Weremijewicz et al. 2016), and the identity of AMF species in the network (Walder et al. 2012).

Seedlings connected to established plants via mycorrhizal networks may have to compete for mycorrhizal-mediated plant nutrients. These nutrients may be preferably allocated to adult plants, as these are the largest carbon provider to the fungus (Pietikäinen \& Kytöviita 2007; Teste et al. 2010; Fellbaum et al. 2014). However, this effect may depend on the species identity of the adult plants as well as of the seedlings, as one study showed that seedlings were not negatively influenced by larger seedlings from heterospecific plants (Moora \& Zobel 1996). These studies indicate that the impact of mycorrhizal networks on seedling establishment may depend on the identity of the seedling. This may be due to differences in the level of colonisation by AMF as for example in grassland systems forbs are often more colonized by AMF than grasses (Cortois et al. 2016). However, it is unknown whether this also results in differential responses of forb and grass seedlings exposed to a common mycorrhizal network.

Most studies testing impacts of mycorrhizal networks on seedlings have been performed in pots or microcosms with few plant species. Moreover, the majority of these studies used sterilized soil inoculated with mycorrhizal isolates, thereby working with a newly assembled mycorrhizal network instead of with a natural and well-developed mycorrhizal network. Inoculation experiments in pots are ideal to obtain fundamental understanding of the plant - AMF interaction, but omit that other microbial species and naturally surrounding vegetation might influence the outcome (Hoeksema 2015; Jin et al. 2017). To our knowledge, there are very few field studies in which the role of mycorrhizal networks on seedling performance has been tested.

The aim of the present study was to test the role of local mycorrhizal networks on seedling performance in the field. Ingrowth cores with fine mesh through which microbes can, but roots cannot grow enabled us to do so by growing plants in situ in mesh cores filled with local field soil, inserted in between intact vegetation. This technique has been introduced by Johnson et al. (2001). We performed our study at a chronosequence of ex-arable fields at the Veluwe in The Netherlands. Chronosequences can serve as a model system to study plant-soil interactions throughout time (Kardol et al. 2006; Hannula et al. 2017; Morriën et al. 2017). In the Veluwe chronosequence it has been found that plant-soil feedback changes 


\section{Chapter 5}

from negative in early to positive in mid-later succession stages, which coincides with an increase of AMF biomass abundance in the soil (Kardol et al. 2006). In this chronosequence, it has also been found that the amount of recent plant derived carbon taken up by mycorrhizal fungi is larger in later succession stages than in early succession stages (Hannula et al. 2017). The higher AMF abundance and increased amount of carbon withdrawn from roots suggest that seedlings in later succession soils might benefit less from the local mycorrhizal network than seedlings in early succession soils. However, individual plant species might respond differently to a mycorrhizal network because plant species can differ in their level of AMF colonisation (Cortois et al. 2016).

We tested the hypotheses that interruption of the mycorrhizal network (1) increases seedling biomass, (2) has a stronger positive effect on seedling biomass in later succession soils than in earlier succession soils, (3) has a negative effect on plants with neutral-positive PSF and a positive effect on plants with negative PSF, and (4) has a negative effect on forbs and a positive effect on grasses. To test these hypotheses we grew 8 common grassland species of 4 forbs and 4 grasses, which have a PSF effect ranging from negative to positive according to an earlier study (Cortois et al. 2016). Seedlings were grown in the field in mesh cores filled with $5 \mathrm{~mm}$ sieved living local field soil to keep the local soil microbial community composition preserved and control for mycorrhizal network effects only. Half of the cores were lifted $3 \mathrm{~cm}$ every three days to interrupt the mycorrhizal hyphae that can connect the seedlings to the surrounding vegetation via a mycorrhizal network, and the other half was left undisturbed as control. After 9 weeks of growth total plant biomass and root colonisation by AMF were quantified to analyse the effect of mycorrhizal network interruption by core lifting on seedling biomass.

\section{Methods}

\section{Locations}

The experiment consisted of plants of 8 different species grown in mesh in-growth cores in 6 fields of different succession age to test the effect of natural mycorrhizal networks in grassland on plant biomass production across a successional gradient. We lifted half the cores $3 \mathrm{~cm}$ every 3 days in order to compare the effect of intact (non-lifted) and interrupted (lifted cores) networks on seedling growth. Uplifting the cores was preferred over rotation because this was more practical than rotation (Johnson et al. 2001). The experiment was conducted on former agricultural fields at the Veluwe (The Netherlands) and included 
vegetation stages from 3 young mid and 3 late mid-succession grasslands. The earliest succession field was abandoned from agricultural practices 11 years ago, the oldest succession field was abandoned 28 years ago (Table S1). The fields were grazed by natural herbivores (hares, rabbits, and deer) and introduced livestock (cows, horses and sheep) at low stocking density. During the experiment $1.2 \mathrm{~m}$ tall fences were placed to exclude the introduced livestock, as they would have trampled and grazed the experimental plants. An overview of plant species that are present in the surrounding vegetation at each field is provided in Supplementary Table 2.

\section{Plant species}

We examined 8 plant species that were grouped according to their functional group (grass or forb) and their PSF effect (based on plant growth response to soil conditioned by conspecifics versus heterospecifics according to Cortois et al. (2016). We included 4 plant species with positive to neutral PSF (the grasses Phleum pratense and Holcus lanatus and the forbs Cirsium oleraceum and Crepis capillaris), and 4 with negative PSF (the grasses Festuca rubra and Bromus hordeaceus and the forbs Achillea millefolium and Geranium molle) (Cortois et al, 2016). Seeds were germinated on glass beads in a growth chamber with day : night temperature of $20 / 10^{\circ} \mathrm{C}$ for $16 / 6 \mathrm{~h}$. Geranium molle seeds were scalped and Bromus hordeaceus seeds were germinated in the dark to speed up germination. As the seeds of the different plant species did not germinate at the same time, early germinating species were kept at $4^{\circ} \mathrm{C}$ until all species had germinated. Seedlings were subsequently transplanted to trays with cells of $2 \times 2 \mathrm{~cm}$ wide and $5 \mathrm{~cm}$ deep, filled with gamma-sterilized soil (25 kGray). Seedlings continued to grow for three more weeks in an unheated greenhouse under natural daylight and temperature conditions until they were transferred to the mesh cores one day before transferring them to the fields.

\section{Experimental setup}

All 8 plant species were exposed to two treatments. The first treatment was with or without lifting the cores to interrupt the mycorrhizal network. The second treatment was field succession age. Thereto the 6 locations were pooled into two categories of succession age: young-mid or late-mid, based on the year since abandonment (Table S1), with three fields in each category. In total the experiment comprised eight plant species x 2 (+/- core lifting) x 8 replicates $=128$ plants per succession age category. At each location all test plant species were present, however not in equal numbers $\left(128 / 3\right.$ locations $\left.=42^{2} / 3\right)$; there were 


\section{Chapter 5}

either 4 of 6 plants per species present yet always half of these being lifted and half not being lifted. For an overview of the number of test plants per functional group and plantsoil feedback type at each location see Supplementary Table S3.

To grow plants with intact and with interrupted mycorrhizal networks we used tubes of 3 $\mathrm{cm}$ diameter and $15 \mathrm{~cm}$ long inserted in newly cored equal sized holes in the field. The wall was made of mesh with $30 \mu \mathrm{m}$ pore size, whereas the bottom was solid (Yuansheng Mesh Co., Ltd. Anping, China). The mesh size ensured that plant roots could not pass, whereas mycorrhizal hyphae and other microbes could enter the cores from the sides (Francis \& Read 1994). The soil inside the tubes that were placed in a particular field had been collected from that specific field two days before placing the tubes in the field. The soil was collected just outside each of the fenced areas. In order to collect the soil, aboveground plant parts were removed and soil was dug up from the top $0-20 \mathrm{~cm}$ layer. The soil was sieved using a $5 \mathrm{~mm}$ mesh to remove large roots and stones, and stored for max 2 days at $5^{\circ} \mathrm{C}$ until used to fill the tubes.

Within each field, tubes were randomly placed in a grid of 4 by 11 plants and spaced $30 \mathrm{~cm}$ apart. A corer of $3 \mathrm{~cm}$ diameter was used to make $14 \mathrm{~cm}$ deep holes in which the tubes were inserted. During the experiment, the vegetation inside the experimental plots, but not the experimental plants themselves, was manually cut back to $10 \mathrm{~cm}$ height every fortnight to prevent that the experimental plants were overgrown by the surrounding vegetation.

After nine weeks the tubes were collected and aboveground plant material was harvested, dried at $70^{\circ} \mathrm{C}$ and weighted. Roots were washed and half of the samples was dried at $70^{\circ} \mathrm{C}$ and weighed, the other half was kept on 70\% alcohol until scoring AMF-colonisation. Afterwards these remaining roots were dried and weighed as well.

\section{$A M F$-scoring}

Roots were stained with ink and vinegar according to Vierheilig et al. (1998). In brief, roots were cleared for $10-15$ minutes in $10 \% \mathrm{KOH}(\mathrm{w} / \mathrm{v})$ in a water bath at $95^{\circ} \mathrm{C}$. Cleared roots were rinsed with tap water and stained for 5 minutes in a $5 \%$ ink-vinegar solution at $95^{\circ} \mathrm{C}$. Stained roots were kept in Petri dishes with demineralised water and some drops of vinegar, until roots were examined. In order to score AMF colonisation random root pieces were placed in 5 lines on a microscope glass. From each line of roots, 20 random observations were made, each resulting in a presence/absence of mycorrhizal structures (arbuscules, 
vesicles, hyphae) and the number of non-mycorrhizal structures (septate fungi, resting spores, and microsclerotia).

\section{Soil analysis}

A subsample of $\pm 50 \mathrm{ml}$ field soil (same soil as used to fill the mesh tubes with) was collected for analysis of C, N and P. Samples were dried at $40^{\circ} \mathrm{C}$ for one week and ground. Phosphorus was measured as P-Olsen and total $\mathrm{N}$ and $\mathrm{C}$ were measured using an element analyser (Thermo flash EA 1112, Thermo Fisher Scientific inc., Waltham, USA).

\section{Data analysis}

Plant biomass (shoot + root dry weight) and mycorrhizal root colonization data were both analysed by linear mixed models. Core lifting, time since abandonment, plant functional group (i.e. grass or forb) and PSF type (negative or neutral to positive) were included as fixed factors, field site and plant species were included as random factors. Model selection was based on the Akaike information criterion (AIC) by manually testing the full model against the reduced model until the lowest AIC value is reached. Biomass data was logtransformed, colonization data was square root transformed. The loss of root biomass for determining AMF colonization was corrected by adding up the average loss per plant species to the root biomass. A third linear mixed model was used to test the effect of AMF colonization on plant biomass. The interaction between plant functional group and time was removed from the analysis because it correlates with mycorrhizal colonisation (Table 1). The correlation between AMF colonization and plant biomass was separately tested for rotated and non-rotated plants using Pearson correlation tests. The treatment effects on the level of root colonization by non-mycorrhizal fungi were analysed using Kruskal-Wallis tests, because normality could not be achieved by transforming the data. In the KruskalWallis test the two levels of succession age (young mid and late mid) were used (Table S1). Visual inspection of the non-mycorrhizal colonisation data showed a possible interaction between plant functional group and PSF, which was subsequently tested by splitting the data into grasses and forbs, and testing whether PSF-types differed in the level of colonisation by non-mycorrhizal fungi. The relation between colonisation of AMF and nonmycorrhizal fungi was analysed by generalized linear model with Poisson distribution. Only plant functional group was included as co-variable because addition of treatment, time, or PSF to the model caused heteroscedasticity, which could not be solved by data transformation. One extreme data point (19 times Cook's distance) was excluded from the 


\section{Chapter 5}

analysis but is shown in the figure between brackets (Fig 3b). To test for differences in the number of seedlings that died in one of the treatment groups (core lifting or control) a Chisquare test was performed.

All analyses were conducted in $\mathrm{R}$ (version.3.3.0). Linear mixed models were fitted with lme4 (Bates et al. 2015), graphs were made in $\mathrm{R}$ with package ggplot2 (Wickham 2009) and modified in Adobe Illustrator (CC 2015).

\section{Results}

Interruption of the AMF network by core lifting significantly reduced mycorrhizal colonisation (Table 1, Fig. 1a). When averaged across all plant species and field sites, core lifting reduced mycorrhizal colonisation of the plant roots from $41 \pm 3 \%$ to $31 \pm 3 \%$. Core lifting significantly increased plant biomass across all plant species (Fig 1b, Table 2). The linear mixed model analysing the relation between AMF colonization and plant biomass showed that percentage mycorrhizal colonisation was negatively correlated to plant biomass at the end of the experiment (Table S4). Further analysis, however, showed that for the plants that were grown in non-lifted cores higher percentage AMF colonisation correlated negatively to plant biomass ( $r=-0.41, P=0.005)$, whereas for the plants grown in lifted cores biomass was not correlated to AMF colonization $(r=0.08, P=0.58)$ (Fig. 1c).

The time since abandonment of arable practices did not significantly affect seedling biomass $\left(\mathrm{F}_{1,4}=1.46, P=0.29\right)$, nor did time since abandonment and core lifting interact in their effect on seedling biomass $\left(\mathrm{F}_{1,190}=0.07, P=0.79\right)$ (Table 2). However, time since abandonment affected mycorrhizal colonisation of grasses and forbs differentially (Table 1). Percentage AMF colonisation of grasses was not influenced by time since abandonment ( $r=0.05, P=0.72$ ), whereas percentage AMF colonisation of forbs decreased from on average $50 \%$ to approximately $25 \%$ with time $(r=-0.43, P=0.002)$ (Fig. 2a). The effect of core lifting on seedling biomass did not interact with PSF type $\left(\mathrm{F}_{1,190}=0.24, P=0.62\right)$ or plant functional group $\left(\mathrm{F}_{1,190}=1.31, P=0.25\right)$ (Table 2$)$.

Plant functional group affected the level of non-AMF colonisation in the plant roots with overall forbs being less colonised by non-mycorrhizal structures than grasses $(P=0.008)$ (Table S5). In addition, forbs with neutral-positive PSF had less non-mycorrhizal colonisation than forbs with negative PSF $(P=0.003)$, whereas there was no such relation for grasses (Fig 2c). 
a)

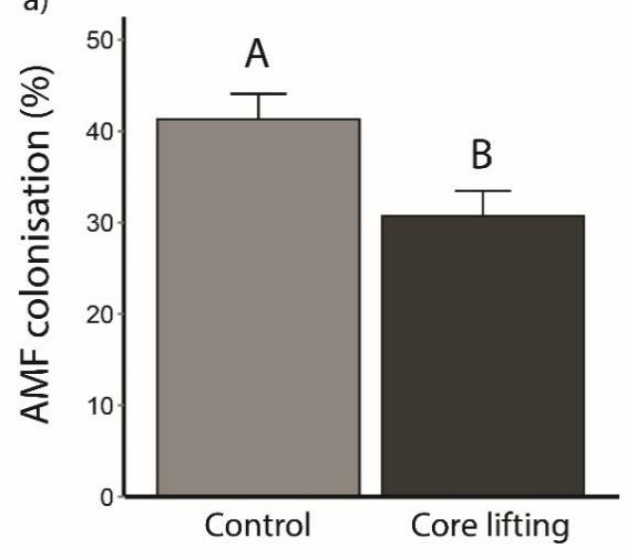

b)

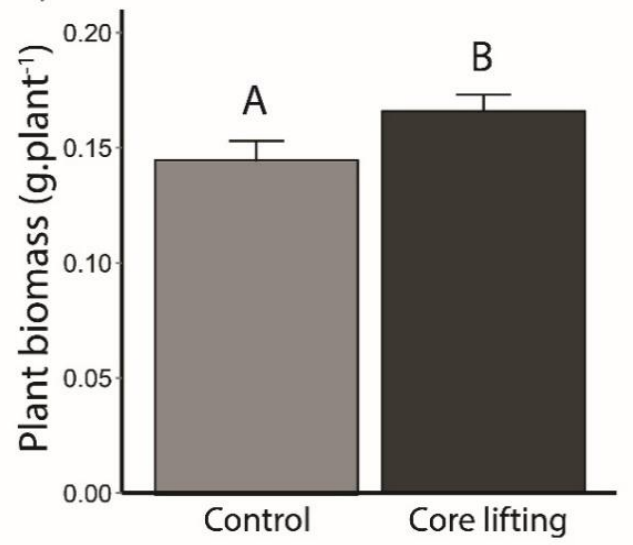

c)

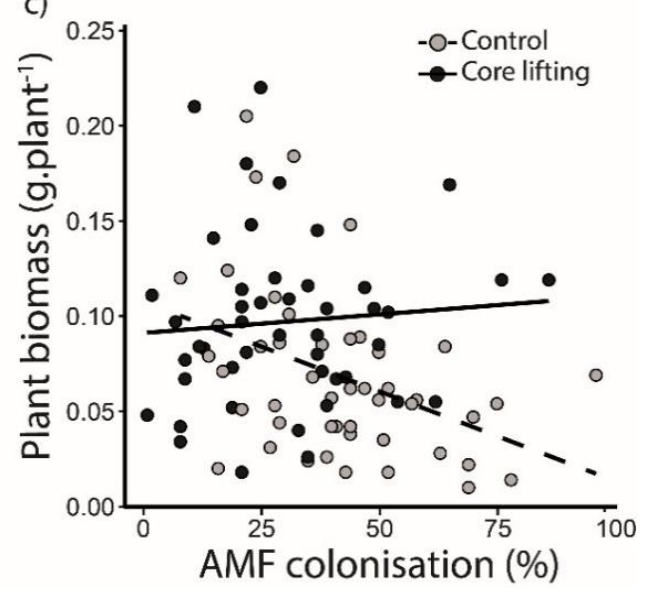

Figure 1 The effect of core lifting on a) mycorrhizal colonisation (mean $\pm \mathrm{SE}) P$ $=0.0005, \mathrm{~b})$ total plant biomass (mean \pm SE) $P=0.002$ and c) on the correlation of plant biomass and mycorrhizal colonisation of plants in lifted cores (black circles, solid line) $(r=-0.41, P=$ 0.005 ) or non-lifted cores (grey circles, dashed line $)(r=0.08, P=0.58)$ Different letters above the bars indicate significant differences between control and core lifted plants. 
a)

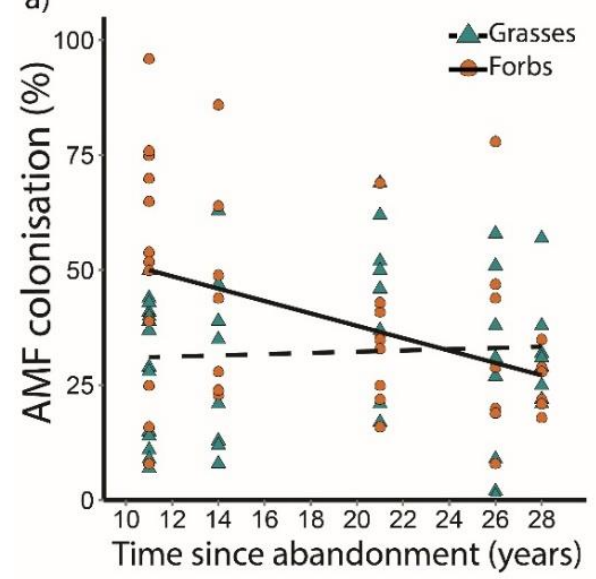

b)

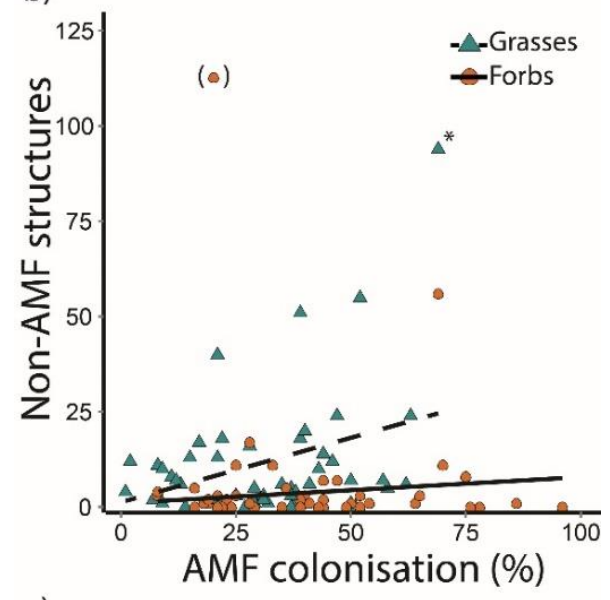

c)

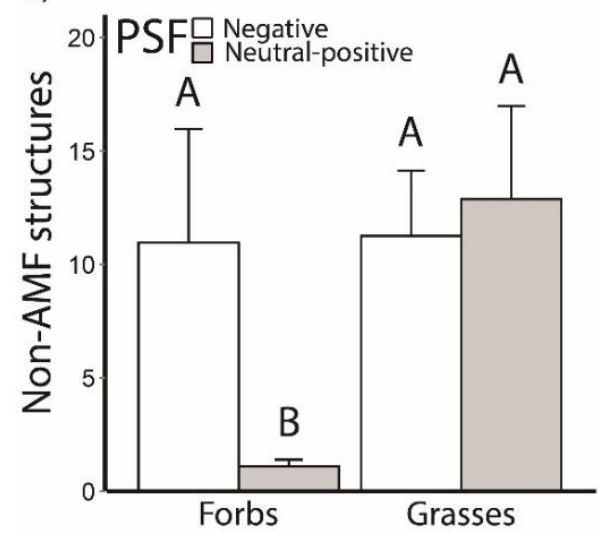

Figure 2 Relation of plant functional group with a) time since abandonment on mycorrhizal colonisation for grasses (green triangle, dashed lines) $(r=0.05, P$ $=0.72$ ) or forbs (orange circle solid line) ( $r=-0.43, P=0.002)$, b) mycorrhizal colonisation on the number of non-AMF structures for grasses (green triangles, dashed line) or forbs (orange circles, solid line). The outlying forb data point () was excluded from analysis and only with the inclusion of the extreme grass data point $*$ the interaction between plant functional group and nonmycorrhizal structures was significant $(P$ $=0.004)$; and $c$ ) the number of nonmycorrhizal structures (mean $\pm \mathrm{SE}$ ) for forbs or grasses per plant-soil feedback type $($ white $=$ negative, grey $=$ neutralpositive). Different letters above the bars indicate significant differences between plant-soil feedback types 
There appeared to be a significant interaction between AMF-colonisation and plant functional group on the abundance of the non-mycorrhizal structures in the roots $(P=$ 0.004), however this significance solely depended on one outlying data point (Table S6, Fig. 2b). When that point was not included in the analysis, significance disappeared $(P=$ $0.35)$.

The numbers of seedlings that did not establish were 21 and 25 for the lifted and non-lifted plants, respectively. These numbers were out of a total of 128 seedlings per group and there was no significantly different mortality between the core lifting treatments $\left(\chi^{2}=0.4542, P=\right.$ 0.797).

\section{Discussion}

We used core lifting as a modified core rotation approach in order to study the effect of plant exposure to surrounding mycorrhizal networks, while keeping the local soil microbial community composition and soil abiotic conditions otherwise the same. Core lifting prevented plant exposure to the mycorrhizal network of the surrounding plant species while allowing for mycorrhizal colonisation within the core. Core lifting is likely to reduce the chance of AMF that are linked to surrounding plant species to enter the core. As expected, root colonisation was lower in lifted than in control cores, which is in line with studies that used core rotation (Zhang et al. 2011; Blanke et al. 2012; Liu et al. 2014). In our experiment, the reduction in AMF colonisation by core lifting was on average $10 \%$ and thereby represents a similar percentage as compared to other studies that used a core rotation approach.

We found that seedling biomass was higher when the mycorrhizal network was interrupted by core lifting than of seedlings which were exposed to surrounding mycorrhizal networks as they were not disturbed, which is in support of our first hypothesis. We also found that root colonisation by AMF negatively correlated with seedling biomass, but only for the seedlings that were grown in the control cores where AMF connections between the soil within and outside the cores were not interrupted. This result suggests that seedlings that are connected to other plants via a mycorrhizal network have reduced benefit compared to seedlings that are colonised by mycorrhiza but are not connected to other plants via the mycorrhizal network. Biomass reduction of seedlings in a mycorrhizal network is presumably due to carbon withdrawal for maintenance and extension of the network with too little returns of plant growth limiting nutrients. 


\section{Chapter 5}

In contrast to our results, several other studies found that mycorrhizal networks support seedling growth (Weremijewicz \& Janos 2013; Liu et al. 2014). In these studies, however, all plants were of similar age, whereas in our field experiment most external plants were older, which may have resulted in asymmetric competition for nutrient benefits between the older plants and the seedlings via the mycorrhizal network (Kytöviita et al. 2003). This is in line with research under highly controlled conditions showing that mycorrhiza supply more nutrients to plants that provide larger amounts of carbon (Kiers et al. 2011; Weremijewicz et al. 2016). Therefore, seedlings that cannot yet fix large amounts of carbon are unlikely to benefit from the mycorrhizal network when they grow in a mixed plant community with older plants.

Plant-plant competition for nutrients might change with succession age of the soil, because both plant and AMF community compositions and soil nutrient levels change during secondary succession (Koziol et al. 2015; Martínez-García et al. 2015; Hannula et al. 2017). We expected that competition between plants exposed to a mycorrhizal network would be greater in later succession fields and therefore biomass of seedlings exposed to the network would be lower in later than in early succession fields. However, we found no evidence that seedlings were differentially affected by the mycorrhizal network in fields of different succession age. The reason for this may be that the youngest field in our study has already been abandoned from agriculture for 11 years, and this might not be young enough to find contrasting results with the older fields. Yet, in contrast to what might be expected, all forb species showed a lower level of colonisation by AMF with increased age of secondary succession of the field site.

All four forbs that were used in our experiment are typical mid succession grassland species, and were expected to be colonised equally well or more in the late-mid succession soils compared to the young-mid succession soils. A reason for reduced AMF colonisation may be that AMF communities from later succession stages might have different host preferences (de León et al. 2016), and might be more efficient or less aggressive colonisers. However, it has been argued that percentage colonisation does not have to be a measure of dependency, or benefit, because of non-linear relations between level of AMF colonization and level of plant growth benefits (Klironomos 2000; De Deyn et al. 2009) so that it is hard to link the reduced level of AMF colonisation in the forbs to implications for plant growth.

Plant functional group is an important predictor of AMF responsiveness; in field studies grass biomass response to AMF is generally lower or even negative compared to forbs as 
shown in several meta-analyses (Hoeksema et al. 2010; Lin et al. 2015). However, we found no evidence that grass and forb seedling growth was affected differentially when the mycorrhizal network was interrupted by core lifting. A reason for this unexpected result may be that the majority of field studies in the meta-analyses of Hoeksema et al. (2010) and Lin et al. (2015) concerned agricultural and forest systems, which are different from seminatural grasslands that have been used in our study. Our fields may have higher plant diversity, different plant identities and, therefore, likely also different AMF community composition (Martínez-García et al. 2015; Vályi et al. 2015) and AMF effect on plant performance (Klironomos et al. 2000; Van der Heijden et al. 2003) than agricultural and forest systems. Moreover, in the meta-analysis studies of Hoeksema et al. (2010) and Lin et al. (2015), effects of the presence of AMF were tested rather than effects of the mycorrhizal network. To our knowledge the present study is the first that specifically analysed the effect of presence/absence of mycorrhizal networks on grasses and forbs grown simultaneously across a gradient of succession grasslands.

We found no support for our hypothesis that plants with neutral to positive PSF would suffer and plant species with negative PSF would benefit from core lifting. There was a trend that grass seedlings with positive PSF were suppressed in control cores, whereas biomass of seedlings with negative PSF were not affected by core lifting. Also in contrast to our expectation, there was no difference in AMF root colonisation of plants with positive and negative PSF, in both lifted and control cores. In the study of Cortois et al. (2016), plants with positive PSF had higher AMF colonisation percentage than plants with negative PSF. Our results are not in line with Cortois et al. (2016) probably because the direction of PSF that develops in the field is not as pronounced as in controlled greenhouse studies due to the relative short growth period of the seedlings, and due to direct and indirect interactions with the surrounding vegetation (Heinze et al. 2016; Schittko et al. 2016).

The majority of the root samples that were analysed for AMF colonisation were also colonized by non-mycorrhizal fungi; these non-mycorrhizal structures could be antagonistic or symbiotic endophytes. The impact of fungal root endophytes other than mycorrhiza is often similar to that of AMF: inducing resistance, reducing stress, and transferring nutrients to the host (Schulz 2006). Non-mycorrhizal structures in roots were lower in absolute number in forbs compared to grasses, which was mainly caused by the relatively low numbers of non-mycorrhizal structures in forbs with neutral-positive PSF. Our results are in line with literature (Weishampel \& Bedford 2006; Mandyam et al. 2012), but it is not yet understood why grasses have more endophyte colonisation than forbs. We observed that 


\section{Chapter 5}

AMF and endophyte colonisation were positively correlated. A similar observation comes from alpine meadows, where a positive correlation was found between mycorrhizal vesicles, which appear in older colonized roots, and dark septate endophytes that were most likely saprophytic in this study (Ruotsalainen et al. 2002). In contrast to our observation, a negative correlation between AMF and non-AMF colonisation was found in wetlands (Weishampel \& Bedford 2006) and forests (Bennett et al. 2017), and it has been suggested that this was due to competition between AMF and endophytes for carbon or for space (Weishampel \& Bedford 2006; Bennett et al. 2017).

In conclusion, we showed that in secondary succession grasslands mycorrhizal networks reduce the biomass of grass and forb seedlings and that increasing AMF colonisation percentage, when exposed to a mycorrhizal network with established plants is a disadvantage in the seedling stage. Time since last agricultural practice did not alter the effect of the mycorrhizal network on seedling growth. We conclude that in a plant community with heterospecific plants and of different age, seedling growth will be suppressed by mycorrhizal connectance to established plants. 


\section{Supporting information}

Table S1. Field information and soil chemical analyses.

\begin{tabular}{|c|c|c|c|c|c|}
\hline Succession age & Location & $\begin{array}{l}\text { Time since } \\
\text { abandonment } \\
\text { (years) }\end{array}$ & Coordinates & $\mathrm{C}: \mathrm{N}$ & P-olsen $(\mathrm{mg} / \mathrm{kg})$ \\
\hline \multirow[t]{3}{*}{ Young mid } & Oud Reemst (OR) & 11 & $\begin{array}{l}\text { N 52'2’27 E } \\
5^{\circ} 48^{\prime} 34\end{array}$ & 19.8 & 147 \\
\hline & Reijerscamp (RC) & 11 & $\begin{array}{l}\text { N 52 }{ }^{\circ} 1^{\prime} 0 \\
5^{\circ} 46^{\prime} 21\end{array}$ & 18.4 & 99.6 \\
\hline & Telefoonweg (TW) & 14 & $\begin{array}{l}\text { N } 52^{\circ} 00^{\prime} 9 \\
5^{\circ} 45^{\prime} 8\end{array}$ & 17.9 & 122 \\
\hline \multirow[t]{3}{*}{ Late mid } & De Mossel (DM) & 21 & $\begin{array}{l}\text { N 52 } 2^{\circ}, 40 \quad \mathrm{E} \\
5^{\circ} 45^{\prime} 8\end{array}$ & 15.5 & 105 \\
\hline & Nieuw Reemst (NR) & 26 & $\begin{array}{l}\text { N 52'2'33 E } \\
5^{\circ} 46^{\prime} 29\end{array}$ & 14.8 & 94.6 \\
\hline & Wolfhezerveld (WV) & 28 & $\begin{array}{l}\text { N 51 }{ }^{\circ} 59^{\prime} 43 \mathrm{E} \\
5^{\circ} 47^{\prime} 24\end{array}$ & 14.4 & 100 \\
\hline
\end{tabular}




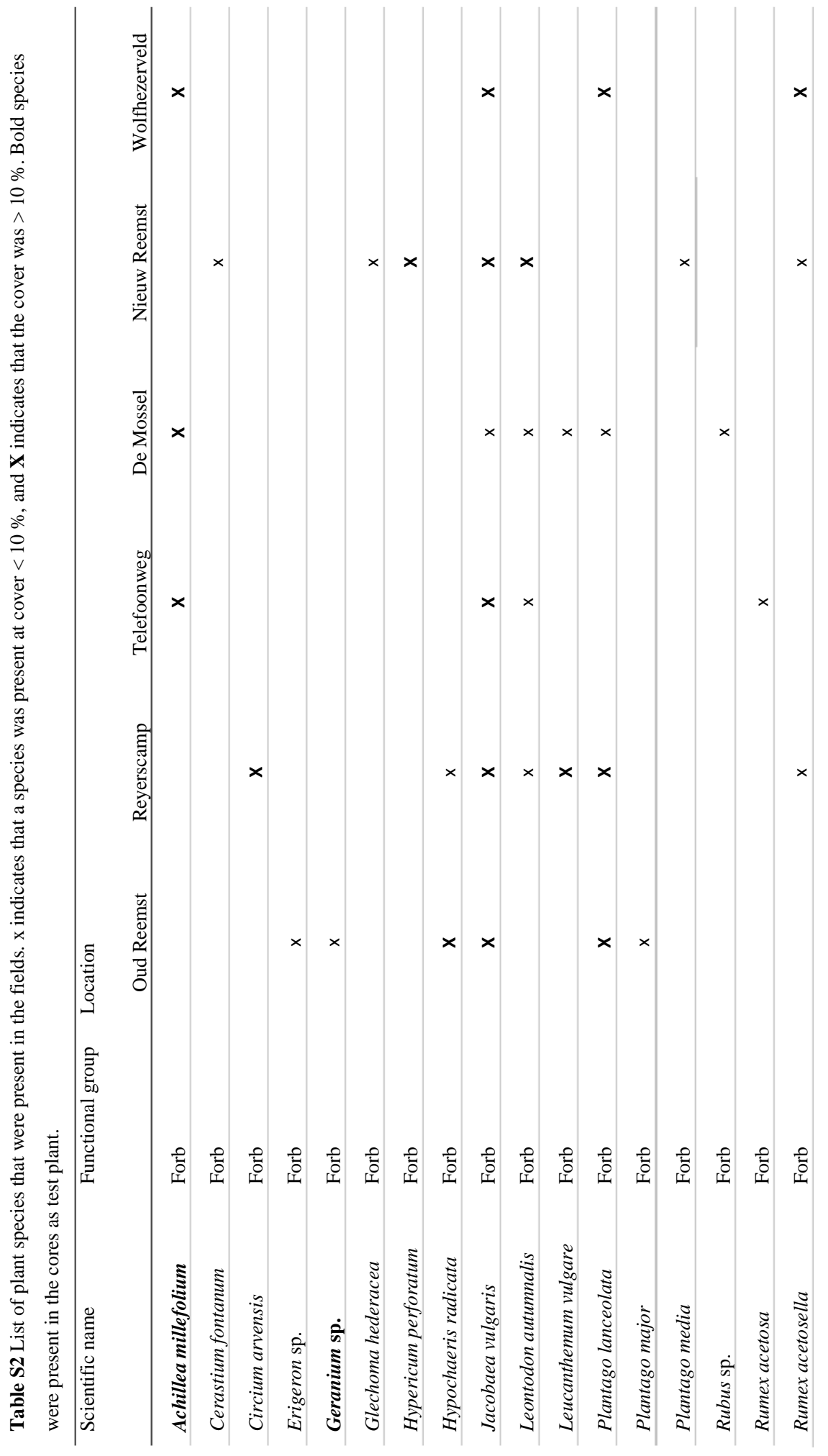


Mycorrhizal networks

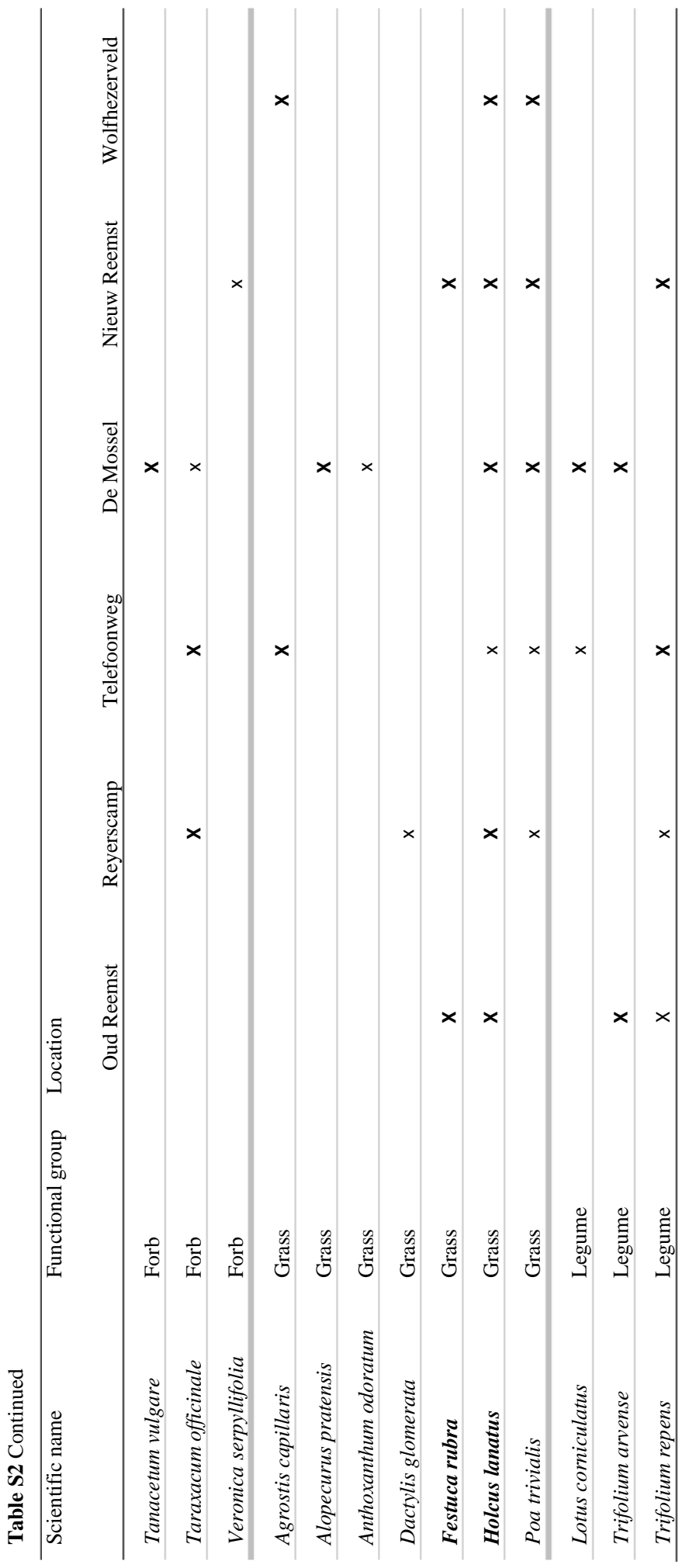


Table S3. Total number of test plants per location. For location abbreviations see Supplementary Table S1, PSF = plant soil feedback type.

\begin{tabular}{|c|c|c|c|c|c|c|}
\hline \multirow{2}{*}{$\begin{array}{l}\text { Succession age } \\
\text { Location }\end{array}$} & \multicolumn{3}{|c|}{ Young mid } & \multicolumn{3}{|c|}{ Late mid } \\
\hline & $\underline{\mathrm{OR}}$ & $\underline{\mathrm{RC}}$ & $\underline{T W}$ & $\underline{\mathrm{DM}}$ & NR & WV \\
\hline Total number of plants & 44 & 42 & 42 & 44 & 42 & 42 \\
\hline Of which: & 22 & 20 & 22 & 20 & 24 & 20 \\
\hline forbs & 22 & 22 & 20 & 24 & 18 & 22 \\
\hline negative PSF & 22 & 22 & 20 & 22 & 20 & 22 \\
\hline neutral-positive PSF & 22 & 20 & 22 & 22 & 22 & 20 \\
\hline
\end{tabular}

Table S4. Total plant biomass response to main effects and interactions of AMF colonisation (Col), time since abandonment (Time), plant functional group (FG), and feedback group of focal plant (PSF) as tested by linear mixed model. Interactions between time and FG were excluded from the analysis because they correlate with colonisation. Presented are the F-test with Kenward-Roger degrees of freedom (Df) and p-values.

\begin{tabular}{llll}
\hline & Df & F-value & p-value \\
\hline AMF colonisation (Col) & 1,46 & 4.56 & $\mathbf{0 . 0 4}$ \\
Time since abandonment (Time) & 1,4 & 0.12 & 0.74 \\
Plant functional group (FG) & 1,3 & 1.74 & 0.29 \\
Feedback type (PSF) & 1,2 & 0.16 & 0.72 \\
Col x Time & 1,79 & 0.05 & 0.82 \\
Col x FG & 1,34 & 2.10 & 0.16 \\
Col x PSF & 1,41 & 2.28 & 0.14 \\
Time x PSF & 1,75 & 0.13 & 0.72 \\
FG x PSF & 1,3 & 0.01 & 0.93 \\
Col x Time x PSF & 1,78 & 0.02 & 0.89 \\
Col x FG x PSF & 1,33 & 0.49 & 0.49 \\
\hline
\end{tabular}


Table S5. Non-mycorrhizal colonisation by septate fungi, resting spores, and microsclerotia in response to main effects of core lifting, succession stage, plant functional group (FG), and feedback group of focal plant (PSF) as tested by Kruskal-Wallis test. Presented are the degrees of freedom (Df), chi square value $\left(X^{2}\right)$ and $\mathrm{p}$-value.

\begin{tabular}{llll}
\hline & Df & $X^{2}$ & p-value \\
\hline Core lifting & 1 & 1.46 & 0.23 \\
Succession stage & 1 & 0.15 & 0.70 \\
Plant functional group & 1 & 7.10 & $\mathbf{0 . 0 0 8}$ \\
Feedback type (PSF) & 1 & 1.25 & 0.26 \\
Grasses x PSF & 1 & 0.07 & 0.79 \\
Forbs x PSF & 1 & 8.61 & $\mathbf{0 . 0 0 3}$ \\
\hline
\end{tabular}

Table S6. Non-mycorrhizal colonisation response for main effects and the interaction of AMF-colonisation and plant functional group (FG) as tested by a generalized linear model. Presented are the degrees of freedom (Df), chi square value $\left(X^{2}\right)$ and p-value.

\begin{tabular}{lccl}
\hline & Df & $X^{2}$ & p-value \\
\hline AMF colonisation & 1,92 & 143 & $<\mathbf{0 . 0 0 0 1}$ \\
Plant functional group (FG) & 1,91 & 295 & $<\mathbf{0 . 0 0 0 1}$ \\
AMF colonisation x FG & 1,90 & 8.27 & $\mathbf{0 . 0 0 4}$ \\
AMF colonisation x FG* & 1,89 & 0.87 & 0.35 \\
\hline
\end{tabular}

Other factors than FG could not be added to the model because this leads to heteroscedasticity which could not be fixed.

* Extreme data point excluded, see material and methods for argumentation. 
Chapter 5 
View over Jena from Der Jenzig. 
Chapter 6

General discussion 


\section{Scope}

Soil biota are critical to the functioning of terrestrial ecosystems. Plant and soil communities are engaged in dynamic interactions, however, plant species richness is declining worldwide and little is known about what this means for soil biodiversity. To date, many studies have focussed on the effects of soil biota on plant performance and plant community dynamics, while the consequences of declining plant species on richness and abundance of soil biota is not yet fully understood. The aim of this thesis was to investigate the impact of plant species richness and community composition on the richness and abundance of soil microbes and nematodes. The work described in this thesis contributes to our understanding of how plant community composition influences the composition of soil biota. I will focus on microbes and nematodes, as these are omnipresent and important components of the soil food web. I especially focussed on effects of plant species and plant trait diversity on microorganisms in and on plant roots and in the bulk soil surrounding the roots and rhizosphere. I also analysed how changes in plant community composition can influence the richness and abundance of biota within different consumer pathways and occupying different trophic positions in the soil food web.

\section{Plant species richness effects on soil biota richness and abundance}

The general assumption that plant species richness positively relates to the richness of nearly all other organisms (Scherber et al. 2010) was not confirmed by the work presented in my thesis (Chapter 2, 3, 4). Instead, I found that the richness of soil fungi, rootassociated fungi and root-associated fungal saprophytes increased with plant species richness, yet, there was no such response observed for fungal plant pathogens, arbuscular mycorrhizal fungi (AMF), bacteria, protists, or various feeding types of nematodes. These results indicate that the various groups of soil biota that I studied have contrasting responses to plant species richness. This finding is in line with another study that simultaneously analysed the response of bacterial and fungal richness to plant species richness (Sugiyama et al. 2008). However, my results are in contrast to those reported by Lange et. al. (2015), who found that in the same Jena Experiment both fungal and bacterial richness were positively related to plant species richness. It should be noted that my studies differed from those of Lange et. al. (2015) in several aspects. For example, we used different molecular tools, which may impact the result found. Another possible explanation for these different findings is that microbial communities need time to develop measurable responses to plant species richness. The samples in the study of Lange et al. (2015) 


\section{Chapter 6}

originate from 2012, which is 2 years later than the collection of my soil samples. The possibility that time after establishing the experiment may influence the strength of plant species richness effects on soil communities is indirectly supported by my finding that fungal diversity in soil was positively related to plant species richness in soil samples taken 8 years after establishment of the Jena Main Experiment but not in samples taken 3 years after establishment of the Jena Trait-Based Experiment (Chapter 2, 4).

Plant species richness not only affected fungal richness, I also found a negative relation with fungal saprophyte abundance and a positive relation with bacterivore and fungal feeding nematodes (Chapter 4). These results show that the effect of plant species richness can influence richness and abundance of soil biota in different directions. The positive effect of plant species richness on fungal saprophytes richness might be caused by an increasing heterogeneity of food sources (Torsvik \& Øvreås 2002). The reduction in fungal saprophyte relative abundance with increased plant species richness could be caused by predation due to fungal feeding nematodes. This is plausible because their abundance increased and their potential to selectively feed on saprophytes. In agricultural soils, it has been shown that larger fungal biomass is positively related to the total abundance of fungal feeding nematodes (Glavatska et al. 2017). In this thesis, I did not analyse fungal biomass and therefore I cannot check whether fungal biomass and fungal feeders are positively related in the Jena Experiment. Although I found that relative abundance of fungal saprophytes reduced, the total abundance of fungi generally increases with plant species richness (Zak et al. 2003; Chung et al. 2007; Lange et al. 2014). Selective feeding of fungal feeding nematodes on fungi can therefore subsequently cause changes in the relative abundance of different groups of soil fungi.

\section{From richness to traits}

There are also other measures of plant community composition that might be better in explaining changes in soil communities than plant species richness. In this thesis I, explored the effects of plant functional group identity, functional diversity (quantified as variation in morphological and development traits) and specific plant traits. This focus is in line with a general shift towards more trait-based approaches in biodiversity studies (Díaz et al. 2003; Baxendale et al. 2014; Bergmann et al. 2016). This trait-based approach can be viewed as an expansion from the use of plant functional groups in which plant species are grouped into plants with roughly similar traits such as grasses, legumes and forbs. I found that the richness and abundance of soil biota in legume plots was different from plots with other 
plant functional groups (Chapter 2). This is not unexpected, as legumes are quite different from other plants because they obtain nitrogen from the atmosphere whereas most other plant species depend on soil mineral nitrogen. Their different demand for soil nutrients might alter the community composition of the microbes with which they are associated and legumes might alter the soil nutrient status to which soil biota respond. In a number of biodiversity studies, legumes had pronounced effects on plant community biomass, nutrient cycling, and decomposition (Hooper \& Vitousek 1998; Spehn et al. 2002; SchererLorenzen 2008). In the subsequent chapters, I show that the community composition of fungi in soil and roots, as well as the community composition of soil nematodes, correlated with the percentage cover of grasses and forbs (Chapter 3, 4), in experiments lacking legume species.

Expanding from plant functional groups to more continuous traits, I found that specific plant traits, such as root length density and flowering start, related to plant morphology and development could explain more variation in soil community composition than plant functional group or plant species richness (Chapter 3, 4). Nearly all specific plant traits affected soil fungal richness, whereas traits did not significantly affect root-associated fungal richness. As suggested earlier in this general discussion, the richness effects in soil are probably related to increased resource heterogeneity (Torsvik \& Øvreås 2002). Specific plant trait effects on richness of root-associated fungi could have been buffered by the host plants (Goldmann et al. 2016). Although specific plant traits did not alter root-associated fungal richness, specific plant traits did alter AMF and saprophyte abundance in roots. The root-associated fungi might be specialists or good competitors that increase in abundance due to resource homogeneity (Tiedje et al. 2001).

Functional diversity explained very little of the variation in microbial and nematode community composition. Reduction in plant species richness or functional diversity does not necessarily remove particular traits from the plant community but rather reduces the variation within particular traits. My findings that functional diversity has relative little impact on soil microbial communities is in line with another study from the Jena TraitBased Experiment (Steinauer et al. 2017) as well as with another study that used perennial grasses to study trait and trait diversity effects on drought resilience (Barkaoui et al. 2016). It has been suggested that functional diversity effects need more time to establish than specific trait effects (Van Ruijven \& Berendse 2009; Ravenek et al. 2014; Barkaoui et al. 2016). In addition, it has also been suggested that trait plasticity should be incorporated into 


\section{Chapter 6}

models that analyse specific trait and trait diversity effects to improve the predictability of plant diversity effects on ecosystem responses (Roscher et al. 2018).

In this thesis, I show that morphological traits and developmental traits can partly predict soil biota community composition, thereby making these traits a useful addition to plant chemical traits. However, the amount of explained variation in my studies remains relatively low. This may be due to the small range of trait values and the relatively few traits that are included in the Jena Trait-Based Experiment. Including a broader range of traits (for example including plant life span, seed morphology characteristics related to dispersal, and germination rate) presents an opportunity to further improve predictions of soil biota community responses.

\section{Soil biota and plant-soil feedback}

I performed a field experiment in which I tested the effect of mycorrhizal networks on seedling performance in a chronosequence of ex-arable fields in the Veluwe area of the Netherlands. I used seedlings of plant species with negative and positive plant-soil feedback, because AMF are thought to contribute to positive plant-soil feedback by increased nutrient supply (Aerts 2002; Read \& Perez-Moreno 2003) and reduced susceptibility to root pathogens (Harrier \& Watson 2004; Wehner et al. 2011). Most plantsoil studies have been performed in pots or microcosms. Hence, little is known about the effects of well-established mycorrhizal networks in the field on seedling performance. In the Veluwe chronosequence it has been found that plant-soil feedback changes from negative in early to positive in mid-later succession stages, which coincides with an increase of AMF biomass abundance in the soil (Kardol et al. 2006). The community composition of non-mycorrhizal fungi (Hannula et al. 2017) and other soil biota (Hannula et al. 2017) were also altered by successional stages of the Veluwe chronosequence.

Despite the changes in soil community composition along the chronosequence, seedling biomass was not altered by the soils of different stages of succession (Chapter 5). These results might indicate that changes in soil biota community composition do not necessarily lead to different feedback effects on the short term. These finding might be due to microbial redundancy (Setälä \& McLean 2004; Delgado-Baquerizo, Giaramida et al. 2016; Nelson et al. 2016; but see Louca et al. 2018), and that mycorrhizal communities, although different in community composition, preserve their general functioning (Gosling et al. 2016). In addition, seedlings might be sensitive to parasitic mycorrhiza (Moora \& Zobel 1996; 
Bennett et al. 2017), and this coincides with the finding that disturbance of the mycorrhizal network connections with the surrounding vegetation, enhanced seedling biomass.

\section{Consequences of soil biodiversity loss}

The consequences of losing microbial and nematode species for the functioning of ecosystems has been examined in a recent controlled experiment, in which species were excluded based on their size (Wagg et al. 2014). It was shown that species loss had profound effects on soil processes as well as on plant species richness, indicating limited redundancy when whole groups of soil biota are excluded based on their size. Another study showed that the loss of fungal saprophytes reduces capabilities for the decomposition of various substrates, although the authors suggest that there is a high degree of redundancy in fungal communities for decomposition processes because fungal diversity effects were only observed at the low end of the diversity gradient (Setälä \& McLean 2004). Despite these studies that show the importance of microbial richness for soil processes, we still no not have a good grasp of how much diversity needs to be maintained to safeguard specific ecosystems functions. Whether species loss has consequences for the community composition of the remaining species and on ecosystem processes might depend on the function of the lost species and on its position in the soil food web. For example, losing plant pathogens might have a larger effect on plant performance than losing saprophytes because pathogens are generally host specific whereas saprophytes have a larger overlap in their resource usage.

Simulation studies of species removal in terrestrial and aquatic food webs revealed that random removal of species has on average little impact on food web stability, whereas removal of a species in a key trophic position destabilises the food web (Dunne et al. 2002). Also in microbial food webs there are so-called keystone taxa, which are relatively well correlated to the abundance of other taxa. It has also been suggested that losses of microbial keystone taxa have profound consequences on microbial community composition (Banerjee et al. 2018). This suggestion could mean that also losses of a few species (when they are key species) can cause alterations in the abundance of remaining species and the ecological roles of the microbial community.

Despite decades of study, the question of the consequences and underlying mechanisms of plant diversity loss for ecosystem functioning remains very timely. A major finding of my 


\section{Chapter 6}

thesis work is that specific plant traits can have much stronger impacts on soil biodiversity than plant species richness per se (Chapters 2, 4). This finding can help to explain why the variation between different plant communities composed of the same number of plant species can be so large, larger than the differences between the average effects of monocultures versus highly species rich plant communities. To illustrate this point, in the Jena Main Experiment on average 90 fungal OTUs were found in soil, with on average 85 OTUs in monocultures and 95 OTUs in the most species-rich plant communities (comprising 60 sown plant species). As a comparison, communities composed of the same number of plant species could differ between 80 and 110 OTUs.

Based on my work and current literature, I propose that changes in plant community composition induce non-random microbial species loss when microbes are associated with specific plant traits (Chapters 3, 4) and plant identity. Therefore, I expect that the observed species loss altered soil ecosystem processes locally. However, in the Jena Experiment, ecosystem functions that involve soil biota do not seem to be hampered by low microbial richness, for example, I found no relation between microbial richness and plant biomass (Chapter 4), plant communities restore fast after flooding (Wright et al. 2017) and bare soil after weeding is quickly covered by new plants. These findings may indicate that there is microbial redundancy in the Jena Experiment. However, one has to keep in mind that as conditions change or more functions are addressed simultaneously more species rich communities, including rare species, can help to safeguard the multiple functions (Isbell et al. 2015; Delgado-Baquerizo, Maestre et al. 2016; Soliveres et al. 2016).

\section{Strengths and limitations of the Jena Experiment for plant-soil biota diversity analyses}

The disadvantage of testing hypotheses in the natural environment is that one cannot control for all disturbances. It is therefore debatable whether field experiments are suitable for analysing refined interactions such as plant-microbe diversity relations. A main advantage of the Jena Experiment for soil microbial research is that there is relatively little variation in $\mathrm{pH}$, which is known to have a major influence on microbial community composition (Lauber et al. 2008; Rousk et al. 2010). Therefore, effects of plant community composition, which might be more subtle, are less likely to be overruled by such abiotic factor. The Jena biodiversity field experiments next to the river Saale, are located on a natural gradient of sandy to clay soils, which is an advantage to test soil structure effects on soil microbial communities. I found that soil texture is an important factor impacting bacterial, fungal and protist community composition (Chapter 2), which is in line with 
other studies that show soil texture to influence microbial richness (Sessitsch et al. 2001), abundance (de Vries et al. 2012), and community composition (Lauber et al. 2013) via pore size and chemistry.

A disadvantage of having this field site next to the river is that the field has been flooded in 2013), which may have caused disturbances in soil. In addition, there is also an elevation gradient in the field, which means that some plots have been flooded more severe than others. Flooding might alter plant community diversity effects on soil microbial communities, but this is not yet confirmed. Plant communities with higher plant species richness were less hampered by flooding than communities low in plant species richness (Wright et al. 2017). It has been suggested that high diversity plots have less dense soils and higher nutrient availability, which supports better plant regrowth (Wright et al. 2017). Soils with poor porosity might have suffered more from anaerobic conditions, which can cause shifts in microbial community composition and activity (van Agtmaal et al. 2015), because activity of many microbes is dependent on oxygen. In addition, anaerobic microbes can produce toxic compounds that reduce the abundance of certain fungal species (Blok et al. 2000). It is therefore more apparent that flooding increases, instead of reduces, the effects of plant species richness on soil microbial communities.

Perhaps, the major disturbance in the Jena Experiment is the impact of weeding. Especially in plots with high weed pressure, the soil becomes more compact because in some cases, the gardeners spend hours removing all the weeds. Plots with low species number have relatively low coverage of target species. These plots therefore have a greater degree of weed pressure and thus a larger level of disturbance due to weeding. Weeding also disturbs the natural dynamics in plant communities by accidental removal of target species and removal of seedlings which are often more difficult to identify. Plant roots are not always removed and this may also influence soil nutrient status via decomposition. This is especially undesirable in cases where the weeds belong to plant functional groups that should not be present in the plot like legumes or grasses. Grass roots, for example, reduce decomposition rates (Chen, Mommer et al. 2017) and legume roots likely influence soil nitrogen status. However, in biodiversity experiments that aim to maintain target species only, weeding is unavoidable. In the Jena Experiment, the researchers keep track of the weeding pressure so that this disturbance can be used as a co-variable in biodiversity studies.

\section{Outlook}




\section{Chapter 6}

In this thesis, I investigated the impact of plant community composition on the richness and abundance of soil microbes and nematodes. This work includes the analyses of large and complex communities. Despite the increasing depth to which microbial communities can be interrogated by high-throughput molecular analysis, the link between species identity and their actual functional contributions to soil ecosystem processes still often remains elusive. Great strides still need to be made in the identification of unknown microbes (Enault et al. 2005; Nguyen et al. 2016) and in tracking down microbial functions.

Ecosystem processes related to plant-soil interactions often cannot be attributed to a single group of soil organisms. Nonetheless, the vast majority of plant-soil interaction studies focus on a single component of the soil biota, despite the fact that methods to do so are generally available, affordable and feasible. The main groups studied are bacteria, fungi and AMF. Recent developments have shown that protists occur at all positions in the soil food web and serve important ecosystem functions (Geisen 2016), but there are still few studies that analysed protist communities simultaneously with other microbial groups (Xiong et al. 2018). Another microbial group that have received relatively little attention are nonmycorrhizal root-endophytes. It has been argued that neutral interactions between endophytes and their host might not exist (Schulz \& Boyle 2005), and that depending on specific environmental conditions root endophytes can act as mutualists as well as antagonists. Identification and characterisation of root-endophytes might also contribute to our understanding of which soil biota are involved in plant-soil feedback.

Soil microbial community composition responds to root exudates (Steinkellner et al. 2007; Sasse et al. 2018) and volatile organic compounds (Schulz-Bohm et al. 2017). The coupling of soil microbial communities to specific patterns of root exudation or volatile organic compounds is an interesting yet little explored field in plant-soil feedback studies. Furthermore, in this thesis, I analysed several aspects of plant community composition based on morphological and development traits and their effect on soil biota. The results from this thesis indicate that further exploring of the relation between plant physiology and microbial community composition can give new insights on plant-soil feedback and other on plant-soil interactions. The findings in this thesis also highlighted that under field conditions plant-soil interactions might be different from pot experiments, which emphasises the importance of testing specific plant-microbe interactions under field conditions before general conclusions are drawn. 
Because soil microbial richness also responds to abiotic environmental factors (Lauber et al. 2008b; Rousk et al. 2010), the search for general patterns of plant species richness on soil microbial richness among different experiments is even more complicated, and might even be an illusion because of the high context dependency (Jonsson \& Wardle 2008; Smith-Ramesh \& Reynolds 2017). There are not yet enough studies available to assess whether plant and microbial species richness are positively related and in which conditions. Other factors, like soil abiotics and climate, can overrule plant diversity effects. Therefore, additional studies dedicated to testing plant species richness effects on multiple groups of soil biota simultaneously are needed, and preferably analysed with high-throughput sequencing techniques to obtain a deep level of coverage of the soil microbial diversity and their link to their functioning. Moreover, in these future studies, environmental factors should be included to elucidate the context dependency. 


\section{References}

Abbas M, Ebeling A, Oelmann Y et al. (2013) Biodiversity Effects on Plant Stoichiometry. PLOS ONE, 8, e58179.

Achatz M, Rillig MC (2014) Arbuscular mycorrhizal fungal hyphae enhance transport of the allelochemical juglone in the field. Soil Biology and Biochemistry, 78, 76-82.

Aerts R (2002) The role of various types of mycorrhizal fungi in nutrient cycling and plant competition. Mycorrhizal Ecology, 157, 117-133.

Aerts R, Chapin FS (1999) The Mineral Nutrition of Wild Plants Revisited: A Reevaluation of Processes and Patterns. Advances in Ecological Research, 30, 1-67.

van Agtmaal M, van Os GJ, Gera Hol WH et al. (2015) Legacy effects of anaerobic soil disinfestation on soil bacterial community composition and production of pathogensuppressing volatiles. Frontiers in Microbiology, 6, 1-12.

Aguilar-Trigueros CA, Rillig MC (2016) Effect of different root endophytic fungi on plant community structure in experimental microcosms. Ecology and Evolution, 6, 81498158.

Allen MF, Boosalis MG (1983) Effects of two species of VA mycorrhizal fungi on drought tolerance of winter wheat. New Phytologist, 93, 67-76.

Anderson MJ, Crist TO, Chase JM et al. (2011) Navigating the multiple meanings of $\beta$ diversity: a roadmap for the practicing ecologist. Ecology Letters, 14, 19-28.

Arndt D, Xia J, Liu Y et al. (2012) METAGENassist: A comprehensive web server for comparative metagenomics. Nucleic Acids Research, 40, 88-95.

Bakker MG, Otto-Hanson L, Lange a. J, Bradeen JM, Kinkel LL (2013) Plant monocultures produce more antagonistic soil Streptomyces communities than highdiversity plant communities. Soil Biology and Biochemistry, 65, 304-312.

Banerjee S, Schlaeppi K, van der Heijden MGA (2018) Keystone taxa as drivers of microbiome structure and functioning. Nature Reviews Microbiology, 1-10.

Barberán A, Mcguire KL, Wolf JA et al. (2015) Relating belowground microbial composition to the taxonomic, phylogenetic, and functional trait distributions of trees in a tropical forest. Ecology Letters, 18, 1397-1405.

Bardgett RD (2002) Causes and consequences of biological diversity in soil. Zoology, 105, 367-374.

Bardgett RD, Mommer L, De Vries FT (2014) Going underground: root traits as drivers of ecosystem processes. Trends in Ecology \& Evolution, 29, 692-699.

Bardgett RD, van der Putten WH (2014) Belowground biodiversity and ecosystem functioning. Nature, 515, 505-511.

Bardgett RD, Wardle DA (2010) Aboveground-Belowground Linkages; Biotic Interactions, Ecosystem Processes, and Global Change. Oxford University Press, New York.

Barkaoui K, Roumet C, Volaire F (2016) Mean root trait more than root trait diversity determines drought resilience in native and cultivated Mediterranean grass mixtures. Agriculture, Ecosystems and Environment, 231, 122-132.

Bartelt-Ryser J, Joshi J, Schmid B, Brandl H, Balser T (2005) Soil feedbacks of plant diversity on soil microbial communities and subsequent plant growth. Perspectives in Plant Ecology, Evolution and Systematics, 7, 27-49.

Bates ST, Berg-Lyons D, Caporaso JG et al. (2011) Examining the global distribution of dominant archaeal populations in soil. The ISME Journal, 5, 908-917.

Bates D, Maechler M, Bolker B, Walker S (2015) Fitting Linear Mixed-Effects Models Using lme4. Journal of Statistical Software, 67, 1-48. 
Baxendale C, Orwin KH, Poly F, Pommier T, Bardgett RD (2014) Are plant-soil feedback responses explained by plant traits? Journal of Physiology, 204, 408-423.

Becklin KM, Hertweck KL, Jumpponen A (2012) Host Identity Impacts Rhizosphere Fungal Communities Associated with Three Alpine Plant Species. Microbial Ecology, 63, 682-693.

Behnke A, Engel M, Christen R et al. (2011) Depicting more accurate pictures of protistan community complexity using pyrosequencing of hypervariable SSU rRNA gene regions. Environmental Microbiology, 13, 340-349.

Bennett AE, Daniell TJ, Öpik M et al. (2013) Arbuscular mycorrhizal fungal networks vary throughout the growing season and between successional stages. PLoS ONE, 8.

Bennett JA, Maherali H, Reinhart KO et al. (2017) Plant-soil feedbacks and mycorrhizal type influence temperate forest population dynamics. Science, 355, 181-184.

Bent SJ, Pierson JD, Forney LJ et al. (2007) Measuring Species Richness Based on Microbial Community Fingerprints: the Emperor Has No Clothes. Applied and Environmental Microbiology, 73, 2399-2401.

Berg G, Smalla K (2009) Plant species and soil type cooperatively shape the structure and function of microbial communities in the rhizosphere. FEMS microbiology ecology, 68, $1-13$.

Bergmann J, Verbruggen E, Heinze J et al. (2016) The interplay between soil structure, roots, and microbiota as a determinant of plant-soil feedback. Ecology and Evolution, 6, 7633-7644.

Bever JD, Mangan SA, Alexander HM (2015) Maintenance of Plant Species Diversity by Pathogens. Annual Review of Ecology, Evolution, and Systematics, 46, 305-325.

Bever JD, Westover KM, Antonovics J (1997) Incorporating the soil community into plant population dynamics: the utility of the feedback approach. Journal of Ecology, 85, $561-573$.

Bezemer TM, Lawson CS, Hedlund K et al. (2006) Plant species and functional group effects on abiotic and microbial soil properties and plant-soil feedback responses in two grasslands. Journal of Ecology, 94, 893-904.

Blanke V, Bassin S, Volk M, Fuhrer J (2012) Nitrogen deposition effects on subalpine grassland: The role of nutrient limitations and changes in mycorrhizal abundance. Acta Oecologica, 45, 57-65.

Blok WJ, Lamers JG, Termorshuizen AJ, Bollen GJ (2000) Control of Soilborne Plant Pathogens by Incorporating Fresh Organic Amendments Followed by Tarping. Phytopathology, 90, 253-259.

De Boer W, Kowalchuk GA, van Veen JA (2006) "Root-Food" and the Rhizosphere Microbial Community Composition. The New Phytologist, 170, 3-6.

Bonkowski M (2004) Protozoa and plant growth: The microbial loop in soil revisited. New Phytologist, 162, 617-631.

Bradford MA (2016) Re-visioning soil food webs. Soil Biology and Biochemistry, 102, 1-3.

Bradford MA, Jones TH, Bardgett RD et al. (2002) Impacts of soil faunal community composition on model grassland ecosystems. Science, 298, 615-618.

Broeckling CD, Broz AK, Bergelson J, Manter DK, Vivanco JM (2008) Root exudates regulate soil fungal community composition and diversity. Applied and Environmental Microbiology, 74, 738-744.

Buée M, de Boer W, Martin F, van Overbeek L, Jurkevitch E (2009) The rhizosphere zoo: An overview of plant-associated communities of microorganisms, including phages, bacteria, archaea, and fungi, and of some of their structuring factors. Plant and Soil, 321, 189-212. 
Buée M, Reich M, Murat C et al. (2009) 454 Pyrosequencing analyses of forest soils reveal an unexpectedly high fungal diversity. New Phytologist, 184, 449-456.

Bulgarelli D, Schlaeppi K, Spaepen S, van Themaat EVL, Schulze-Lefert P (2013) Structure and Functions of the Bacterial Microbiota of Plants. Annual Review of Plant Biology, 64, 807-838.

Burns JH, Anacker BL, Strauss SY, Burke DJ (2015) Soil microbial community variation correlates most strongly with plant species identity, followed by soil chemistry, spatial location and plant genus. AoB PLANTS, 7, 1-10.

Cantarel AAM, Pommier T, Desclos-Theveniau M et al. (2015) Using plant traits to explain plant-microbe relationships involved in nitrogen acquisition. Ecology, 96, 788-799.

Cardinale BJ, Duffy JE, Gonzalez A et al. (2012) Biodiversity loss and its impact on humanity. Nature, 486, 59-67.

Carmona D, Lajeunesse MJ, Johnson MTJ (2011) Plant traits that predict resistance to herbivores. Functional Ecology, 25, 358-367.

Van Cauwenberghe J, Michiels J, Honnay O (2015) Effects of local environmental variables and geographical location on the genetic diversity and composition of Rhizobium leguminosarum nodulating Vicia cracca populations. Soil Biology and Biochemistry, 90, 71-79.

Cavicchioli R, Curmi PMG, Saunders N, Thomas T (2003) Pathogenic archaea: Do they exist? BioEssays, 25, 1119-1128.

Chakraborty S (2001) grassland plant diseases: management and control. In: Proceedings of the XIX International Grassland Congress: Grassland ecosystems: an outlook into the 21 st century, pp. 223-230.

Chen M, Chen B, Marschner P (2008) Plant growth and soil microbial community structure of legumes and grasses grown in monoculture or mixture. Journal of Environmental Sciences, 20, 1231-1237.

Chen H, Mommer L, van Ruijven J et al. (2017) Plant species richness negatively affects root decomposition in grasslands. Journal of Ecology, 105, 209-218.

Chen YL, Xu T Le, Veresoglou SD et al. (2017) Plant diversity represents the prevalent determinant of soil fungal community structure across temperate grasslands in northern China. Soil Biology and Biochemistry, 110, 12-21.

Chung H, Zak DR, Reich PB, Ellsworth DS (2007) Plant species richness, elevated $\mathrm{CO}_{2}$, and atmospheric nitrogen deposition alter soil microbial community composition and function. Global Change Biology, 13, 980-989.

Clay K (1999) Fungal Endophyte Symbiosis and Plant Diversity in Successional Fields. Science, 285, 1742-1744.

Cole JR, Wang Q, Fish JA et al. (2014) Ribosomal Database Project: Data and tools for high throughput rRNA analysis. Nucleic Acids Research, 42, 633-642.

Compant S, Duffy B, Nowak J, Cle C, Barka E a (2005) Use of Plant Growth-Promoting Bacteria for Biocontrol of Plant Diseases : Principles, Mechanisms of Action, and Future Prospects MINIREVIEW Use of Plant Growth-Promoting Bacteria for Biocontrol of Plant Diseases : Principles, Mechanisms of Action, and. Applied and environmental microbiology, 71, 4951-4959.

Cornwell WK, Cornelissen JHC, Amatangelo K et al. (2008) Plant species traits are the predominant control on litter decomposition rates within biomes worldwide. Ecology Letters, 11, 1065-1071.

Cortez J, Garnier E, Pérez-Harguindeguy N, Debussche M, Gillon D (2007) Plant traits, litter quality and decomposition in a Mediterranean old-field succession. Plant and 
Soil, 296, 19-34.

Cortois R, Schröder-Georgi T, Weigelt A, van der Putten WH, De Deyn GB (2016) Plantsoil feedbacks: role of plant functional group and plant traits. Journal of Ecology, 104, 1608-1617.

Cortois R, Veen GFC, Duyts H et al. (2017) Possible mechanisms underlying abundance and diversity responses of nematode communities to plant diversity. Ecosphere, $\mathbf{8}$, e01719.

Dassen S, Cortois R, Martens H et al. (2017) Differential responses of soil bacteria, fungi, archaea and protists to plant species richness and plant functional group identity. Molecular Ecology, 26, 4085-4098.

Delgado-Baquerizo M, Giaramida L, Reich PB et al. (2016) Lack of functional redundancy in the relationship between microbial diversity and ecosystem functioning. Journal of Ecology, 104, 936-946.

Delgado-Baquerizo M, Maestre FT, Reich PB et al. (2016) Microbial diversity drives multifunctionality in terrestrial ecosystems. Nature Communications, 7, 10541.

Delgado-Baquerizo M, Powell JR, Hamonts K et al. (2017) Circular linkages between soil biodiversity, fertility and plant productivity are limited to topsoil at the continental scale. New Phytologist, 215, 1186-1196.

De Deyn GB, Biere A, van der Putten WH, Wagenaar R, Klironomos JN (2009) Chemical defense, mycorrhizal colonization and growth responses in Plantago lanceolata L. Oecologia, 160, 433-442.

De Deyn GB, Quirk H, Bardgett RD (2011) Plant species richness, identity and productivity differentially influence key groups of microbes in grassland soils of contrasting fertility. Biology Letters, 7, 75-78.

De Deyn GB, Raaijmakers CE, Van Ruijven J, Berendse F, Van Der Putten WH (2004) Plant species identity and diversity effects on different trophic levels of nematodes in the soil food web. Oikos, 106, 576-586.

Díaz S, Cabido M (2001) Vive la différence: Plant functional diversity matters to ecosystem processes. Trends in Ecology and Evolution, 16, 646-655.

Díaz S, Symstad AJ, Chapin FS, Wardle DA, Huenneke LF (2003) Functional diversity revealed by removal experiments. Trends in Ecology and Evolution, 18, 140-146.

Dignam BEA, O'Callaghan M, Condron LM et al. (2016) Challenges and opportunities in harnessing soil disease suppressiveness for sustainable pasture production. Soil Biology and Biochemistry, 95, 100-111.

Dodt M, Roehr J, Ahmed R, Dieterich C (2012) FLEXBAR_Flexible Barcode and Adapter Processing for Next-Generation Sequencing Platforms. Biology, 1, 895-905.

Dudenhöffer JH, Ebeling A, Klein AM, Wagg C (2018) Beyond biomass: Soil feedbacks are transient over plant life stages and alter fitness. Journal of Ecology, 106, 230 241.

Dumbrell AJ, Ashton PD, Aziz N et al. (2011) Distinct seasonal assemblages of arbuscular mycorrhizal fungi revealed by massively parallel pyrosequencing. New Phytologist, 190, 794-804.

Dumbrell AJ, Nelson M, Helgason T, Dytham C, Fitter AH (2010) Relative roles of niche and neutral processes in structuring a soil microbial community. The ISME Journal, 4, 337-345.

Dunne JA, Williams RJ, Martinez ND (2002) Network structure and biodiversity loss in food webs: robustness increase with connectance. Ecology Letters, 5, 558-567.

Ebeling A, Pompe S, Baade J et al. (2014) A trait-based experimental approach to understand the mechanisms underlying biodiversity-ecosystem functioning 
relationships. Basic and Applied Ecology, 15, 229-240.

Edgar RC (2010) Search and clustering orders of magnitude faster than BLAST. Bioinformatics, 26, 2460-2461.

Edgar RC, Haas BJ, Clemente JC, Quince C, Knight R (2011) UCHIME improves sensitivity and speed of chimera detection. Bioinformatics (Oxford, England), 27, 2194-200.

Ehrenfeld JG, Ravit B, Elgersma K (2005) Feedback in the Plant-Soil System. Annual Review of Environment and Resources, 30, 75-115.

Eisenhauer N, Bessler H, Engels C et al. (2010) Plant diversity effects on soil microorganisms support the singular hypothesis. Ecology, 91, 485-96.

Eisenhauer N, Migunova VD, Ackermann M, Ruess L, Scheu S (2011) Changes in Plant Species Richness Induce Functional Shifts in Soil Nematode Communities in Experimental Grassland. PLoS ONE, 6, e24087.

Enault F, Suhre K, Claverie JM (2005) Phydbac "gene function predictor": A gene annotation tool based on genomic context analysis. BMC Bioinformatics, 6, 1-10.

Erktan A, Cécillon L, Graf F et al. (2016) Increase in soil aggregate stability along a Mediterranean successional gradient in severely eroded gully bed ecosystems: combined effects of soil, root traits and plant community characteristics. Plant and Soil, 398, 121-137.

Ettema CH, Wardle DA (2002) Spatial soil ecology. Trends in Ecology and Evolution, 17, 177-183.

Faucon MP, Houben D, Lambers H (2017) Plant Functional Traits: Soil and Ecosystem Services. Trends in Plant Science, 22, 385-394.

Fellbaum CR, Mensah JA, Cloos AJ et al. (2014) Fungal nutrient allocation in common mycorrhizal networks is regulated by the carbon source strength of individual host plants. New Phytologist, 203, 646-656.

Fierer N, Jackson RB (2006) The diversity and biogeography of soil bacterial communities. Proceedings of the National Academy of Sciences of the United States of America, 103, 626-631.

Fierer N, Schimel JP, Holden PA (2003) Variations in microbial community composition through two soil depth profiles. Soil Biology and Biochemistry, 35, 167-176.

Fischer C, Tischer J, Roscher C et al. (2015) Plant species diversity affects infiltration capacity in an experimental grassland through changes in soil properties. Plant and Soil, 397, 1-16.

Francis R, Read DJ (1994) The contributions of mycorrhizal fungi to the determination of plant community structure. Plant and Soil, 159, 11-25.

Francis R, Read DJ (1995) Mutualism and antagonism in the mycorrhizal symbiosis, with special reference to impacts on plant community structure. Canadian Journal of Botany, 73, 1301-1309.

Freschet GT, Cornwell WK, Wardle D a. et al. (2013) Linking litter decomposition of above- and below-ground organs to plant-soil feedbacks worldwide. Journal of Ecology, 101, 943-952.

Gage DJ (2004) Infection and invasion of roots by symbiotic, nitrogen-fixing rhizobia during nodulation of temperate legumes. Microbiology and molecular biology reviews: $M M B R, \mathbf{6 8}, 280-300$.

Garbeva P, van Elsas JD, van Veen J a. (2008) Rhizosphere microbial community and its response to plant species and soil history. Plant and Soil, 302, 19-32.

Geisen S (2016) The bacterial-fungal energy channel concept challenged by enormous functional versatility of soil protists. Soil Biology and Biochemistry, 102, 22-25. 
Geisen S, Kostenko O, Cnossen MC et al. (2017) Seed and root endophytic fungi in a range expanding and a related plant species. Frontiers in Microbiology, 8, 1-11.

Georg Niedrist, Tasser E, Lüth C, Dalla Via J, Tappeiner U (2009) Plant Diversity Declines with Recent Land Use Changes in European Alps. , 202, 195-210.

Glaser K, Kuppardt A, Boenigk J et al. (2015) The influence of environmental factors on protistan microorganisms in grassland soils along a land-use gradient. Science of the Total Environment, 537, 33-42.

Glavatska O, Müller K, Butenschoen O et al. (2017) Disentangling the root- and detritusbased food chain in the micro-food web of an arable soil by plant removal. PLoS ONE, 12, 1-18.

Goldmann K, Schröter K, Pena R et al. (2016) Divergent habitat filtering of root and soil fungal communities in temperate beech forests. Scientific Reports, 6, 1-10.

Gosling P, Jones J, Bending GD (2016) Evidence for functional redundancy in arbuscular mycorrhizal fungi and implications for agroecosystem management. Mycorrhiza, 26, $77-83$.

Griffiths BS, Ritz K, Bardgett RD et al. (2000) Ecosystem Response of Pasture Soil Communities to Fumigation-Indicuded Microbial Diversity Reudctions: An Examination of the Biodiversity - Ecosystem Function Relationship. Oikos, 90, 279294.

Grime JP (2002) Declining plant diversity: Empty niches or functional shifts? Journal of Vegetation Science, 13, 457-460.

Grüter D, Schmid B, Brandl H (2006) Influence of plant diversity and elevated atmospheric carbon dioxide levels on belowground bacterial diversity. BMC microbiology, 6, 68 .

Guiz J, Ebeling A, Eisenhauer N et al. (2018) Interspecific competition alters leaf stoichiometry in 20 grassland species. Oikos, 1-12.

Güsewell S, Gessner MO (2009) N:P ratios influence litter decomposition and colonization by fungi and bacteria in microcosms. Functional Ecology, 23, 211-219.

Gweon HS, Oliver A, Taylor J et al. (2015) PIPITS: An automated pipeline for analyses of fungal internal transcribed spacer sequences from the Illumina sequencing platform. Methods in Ecology and Evolution, 6, 973-980.

Gyssels G, Poesen J, Bochet E, Li Y (2005) Impact of plant roots on the resistance of soils to erosion by water: A review. Progress in Physical Geography, 29, 189-217.

Hannula SE, Boschker HTS, de Boer W, van Veen JA (2012) 13C pulse-labeling assessment of the community structure of active fungi in the rhizosphere of a genetically starch-modified potato (Solanum tuberosum) cultivar and its parental isoline. New Phytologist, 194, 784-799.

Hannula SE, Morriën E, de Hollander M et al. (2017) Shifts in rhizosphere fungal community during secondary succession following abandonment from agriculture. The ISME Journal, 1-11.

Harrier LA, Watson CA (2004) The potential role of arbuscular mycorrhizal(AM) fungi in the bioprotection of plants against soil-borne pathogens in organic and/or other sustainable farming systems. Pest Management Science, 60, 149-157.

Hart MM, Reader RJ, Klironomos JN (2003) Plant coexistence mediated by arbuscular mycorrhizal fungi. Trends in Ecology \& Evolution, 18, 418-423.

Hättenschwiler S, Tiunov A V., Scheu S (2005) Biodiversity and Litter Decomposition in Terrestrial Ecosystems. Annual Review of Ecology, Evolution, and Systematics, 36, 191-218.

Hector A (1999) Plant Diversity and Productivity Experiments in European Grasslands. Science, 286, 1123-1127. 
Hedlund K, Santa Regina I, van der Putten WH (2003) Plant species diversity, plant biomass and responses of the soil community on abandoned land across Europe: idiosyncracy or above-belowground time lags. Oikos, 1, 45-58.

Van der Heijden MGA, Bakker R, Verwaal J et al. (2006) Symbiotic bacteria as a determinant of plant community structure and plant productivity in dune grassland. FEMS Microbiology Ecology, 56, 178-187.

Van der Heijden MGA, Bardgett RD, Van Straalen NM (2008) The unseen majority: Soil microbes as drivers of plant diversity and productivity in terrestrial ecosystems. Ecology Letters, 11, 296-310.

Van der Heijden MGA, Horton TR (2009) Socialism in soil? the importance of mycorrhizal fungal networks for facilitation in natural ecosystems. Journal of Ecology, 97, 11391150.

Van der Heijden MGA, Klironomos JN, Ursic M et al. (1998) Mycorrhizal fungal diversity determines plant biodiversity, ecosystem variability and productivity. Nature, 396, 69-72.

van der Heijden MGA, Schlaeppi K (2015) Root surface as a frontier for plant microbiome research: Fig. 1. Proceedings of the National Academy of Sciences, 112, 2299-2300.

Van der Heijden MGA, Wiemken A, Sanders IR, Wiemken A, Sanders IR (2003) Different arbuscular mycorrhizal fungi alter coexistence and resource distribution between cooccurring plant. , 157, 569-578.

Heinze J, Sitte M, Schindhelm A, Wright J, Joshi J (2016) Plant-soil feedbacks: a comparative study on the relative importance of soil feedbacks in the greenhouse versus the field. Oecologia, 181, 559-569.

Helios De Rosario-Martinez (2015) phia: Post-Hoc Interaction Analysis.

Hendriks M, Mommer L, de Caluwe H et al. (2013) Independent variations of plant and soil mixtures reveal soil feedback effects on plant community overyielding. Journal of Ecology, 101, 287-297.

Hiiesalu I, Pärtel M, Davison J et al. (2014) Species richness of arbuscular mycorrhizal fungi: Associations with grassland plant richness and biomass. New Phytologist, 203, 233-244.

Hodge A (2004) The plastic plant: Root responses to heterogeneous supplies of nutrients. New Phytologist, 162, 9-24.

Hoeksema JD (2015) Experimentally testing effects of mycorrhizal networks on plant ecology and distinguishing among mechanisms. In: Mycorrhizal Networks (ed Horton TR), pp. 255-277. Springer, Ecological Studies 224.

Hoeksema JD, Chaudhary VB, Gehring CA et al. (2010) A meta-analysis of contextdependency in plant response to inoculation with mycorrhizal fungi. Ecology Letters, 13, 394-407.

Hol WHG, Bezemer TM, Biere A (2013) Getting the ecology into interactions between plants and the plant growth-promoting bacterium Pseudomonas fluorescens.

Frontiers in Plant Science, 4, 1-9.

de Hollander M (2016) GitLab Community Edition: Open Source Software to Collaborate on Code [Online]. Available at: https://gitlab.bioinf.nioo.knaw.nl/ amplicon metagenomics/illumina_paired_end.

Hooper DU, Dukes JS (2004) Overyielding among plant functional groups in a long-term experiment. Ecology Letters, 7, 95-105.

Hooper DU, F. S. Chapin I, Ewel JJ et al. (2005) Effects of Biodiversity on Ecosystem

Functioning: A Consensus of Current Knowledge. Ecological Monographs, 75, 3-35.

Hooper DU, Vitousek PM (1998) Effects of plant composition and diversity on nutrient 
cycling. Ecological Monographs, 68, 121-149.

Hunt HW, Wall DH (2002) Modelling the effects of loss of soil biodiversity on ecosystem function. Global Change Biology, 8, 33-50.

Hunter S, Corbett M, Denise H et al. (2014) EBI metagenomics - A new resource for the analysis and archiving of metagenomic data. Nucleic Acids Research, 42, 600-606.

Isbell F, Adler PR, Eisenhauer N et al. (2017) Benefits of increasing plant diversity in sustainable agroecosystems. Journal of Ecology, 105, 871-879.

Isbell F, Craven D, Connolly J et al. (2015) Biodiversity increases the resistance of ecosystem productivity to climate extremes. Nature, 526, 574-577.

Jansa J, Smith FA, Smith SE (2008) Are there benefits of simultaneous root colonization by different arbuscular mycorrhizal fungi? New Phytologist, 177, 779-789.

Jin L, Wang Q, Wang Q, Wang X, Gange AC (2017) Mycorrhizal-induced growth depression in plants. Symbiosis, 72, 81-88.

Jing J, Bezemer TM, van der Putten WH (2015) Complementarity and selection effects in early and mid-successional plant communities are differentially affected by plant-soil feedback. Journal of Ecology, 103, 641-647.

Johnson D, Gilbert L (2015) Interplant signalling through hyphal networks. New Phytologist, 205, 1448-1453.

Johnson NC, Graham JH, Smith FA (1997) Functioning of mycorrhizal associations along the mutualism - parasitism continuum. New Phytologist, 575-585.

Johnson D, Leake JR, Read DJ (2001) Novel in-growth core system enables functional studies of grassland mycorrhizal mycelial networks. New Phytologist, 152, 555-562.

Jonsson M, Wardle DA (2008) Context dependency of litter-mixing effects on decomposition and nutrient release across a long-term chronosequence. Oikos, 117, 1674-1682.

Jumpponen A, Jones KL, Blair J (2010) Vertical distribution of fungal communities in tallgrass prairie soil. Mycologia, 102, 1027-1041.

Kardol P, Bezemer MT, van der Putten WH (2006) Temporal variation in plant-soil feedback controls succession. Ecology Letters, 9, 1080-1088.

Kardol P, De Deyn GB, Laliberté E, Mariotte P, Hawkes CV (2013) Biotic plant-soil feedbacks across temporal scales. Journal of Ecology, 101, 309-315.

Kardol P, Veen GF (Ciska), Teste FP, Perring MP (2015) Peeking into the black box: a trait- based approach to predicting plant - soil feedback. New Phytologist, 206, 1-4.

Ke P, Miki T, Ding T et al. (2015) The soil microbial community predicts the importance of plant traits in plant - soil feedback. New Phytologist, 3, 329-341.

Kier G, Mutke J, Dinerstein E et al. (2005) Global patterns of plant diversity and floristic knowledge. Journal of Biogeography, 32, 1107-1116.

Kiers ET, Duhamel M, Beesetty Y et al. (2011) Mycorrhizal Symbiosis. 333, 880-883.

Klironomos JN (2000) Host-specificity and functional diversity among arbuscular mycorrhizal fungi. Microbial biosystems: New frontiers, 845-851.

Klironomos JN (2003) Variation in plant response to native and exotic arbuscular mycorrhizal fungi. Ecology, 84, 2292-2301.

Klironomos J, McCune J, Hart M, Neville J (2000) The influence of arbuscular mycorrhizae on the relationship between plant diversity and productivity. Ecology Letters, 3, 137-141.

König S, Wubet T, Dormann CF et al. (2010) TaqMan Real-Time PCR Assays To Assess Arbuscular Mycorrhizal Responses to Field Manipulation of Grassland Biodiversity: Effects of Soil Characteristics, Plant Species Richness, and Functional Traits. Applied and Environmental Microbiology, 76, 3765-3775. 
Korthals GW, Smilauer P, Van Dijk C, Van Der Putten WH (2001) Linking above- and below-ground biodiversity: abundance and trophic complexity in soil as a response to experimental plant communities on abandoned arable land. Functional Ecology, 15, 506-514.

Köster J, Rahmann S (2012) Snakemake--a scalable bioinformatics workflow engine. Bioinformatics (Oxford, England), 28, 2520-2.

Kowalchuk GA, Buma DS, de Boer W, Klinkhamer PGL, van Veen JA (2002) Effects of above-ground plant species composition and diversity on the diversity of soil-borne microorganisms. International Journal of General and Molecular Microbiology, 81, 509-520.

Koziol L, Bever JD, Hawkes C V. (2015) Mycorrhizal response trades off with plant growth rate and increases with plant successional status. Ecology, 96, 1768-1774.

Kristin A, Miranda H (2013) The root microbiota-a fingerprint in the soil? Plant and Soil, 370, 671-686.

Krumins JA, Oevelen D Van, Bezemer TM, Deyn GB De, Hol WHG (2013) Soil and Freshwater and Marine Sediment Food Webs: Their Structure and Function. BioScience, 63, 35-42.

Kulmatiski a., Beard KH, Heavilin J (2012) Plant-soil feedbacks provide an additional explanation for diversity-productivity relationships. Proceedings of the Royal Society B: Biological Sciences, 279, 3020-3026.

Kulmatiski A, Beard KH, Stevens JR, Cobbold SM (2008) Plant-soil feedbacks: a metaanalytical review. Ecology Letters, 11, 980-992.

Kuramae EE, Verbruggen E, Hillekens R et al. (2013) Tracking fungal community responses to maize plants by DNA- and RNA-based pyrosequencing. PloS one, $\mathbf{8}$, e69973.

Kytöviita MM, Vestberg M, Tuomi J (2003) A test of mutual aid in common mycorrhizal networks: Established vegetation negates benefit in seedlings. Ecology, 84, 898-906.

Lange M, Eisenhauer N, Sierra CA et al. (2015) Plant diversity increases soil microbial activity and soil carbon storage. Nature communications, 6, 6707.

Lange M, Habekost M, Eisenhauer N et al. (2014) Biotic and Abiotic Properties Mediating Plant Diversity Effects on Soil Microbial Communities in an Experimental Grassland. PLoS ONE, 9, e96182.

Lauber CL, Ramirez KS, Aanderud Z, Lennon J, Fierer N (2013) Temporal variability in soil microbial communities across land-use types. The ISME Journal, 7, 1641-1650.

Lauber CL, Strickland MS, Bradford MA, Fierer N (2008) The influence of soil properties on the structure of bacterial and fungal communities across land-use types. Soil Biology and Biochemistry, 40, 2407-2415.

LeBlanc N, Kinkel LL, Kistler HC (2015) Soil Fungal Communities Respond to Grassland Plant Community Richness and Soil Edaphics. Microbial Ecology, 70, 188-195.

Ledeganck P, Nijs I, Beyens L (2003) Plant functional group diversity promotes soil protist diversity. Protist, 154, 239-249.

Leff JW, Bardgett RD, Wilkinson A et al. (2018) Predicting the structure of soil communities from plant community taxonomy, phylogeny, and traits. ISME Journal, $1-12$.

Legay N, Baxendale C, Grigulis K et al. (2014) Contribution of above- and below-ground plant traits to the structure and function of grassland soil microbial communities. Annals of Botany, 114, 1011-1021.

Lekberg Y, Bever JD, Bunn RA et al. (2018) Relative importance of competition and plantsoil feedback, their synergy, context dependency and implications for coexistence. 
Ecology Letters, 1-14.

Lekberg Y, Gibbons SM, Rosendahl S, Ramsey PW (2013) Severe plant invasions can increase mycorrhizal fungal abundance and diversity. The ISME Journal, 7, 14241433.

Lekberg Y, Koide RT (2005) Is plant performance limited by abundance of arbuscular mycorrhizal fungi? A meta analysis of studies published between 1988 and 2003. New Phytologist, 168, 189-204.

de León DG, Moora M, Öpik M et al. (2016) Symbiont dynamics during ecosystem succession: Co-occurring plant and arbuscular mycorrhizal fungal communities. FEMS Microbiology Ecology, 92, 1-9.

Lin G, McCormack ML, Guo D (2015) Arbuscular mycorrhizal fungal effects on plant competition and community structure. Journal of Ecology, 103, 1224-1232.

Lipowsky A, Roscher C, Schumacher J et al. (2015) Plasticity of functional traits of forb species in response to biodiversity. Perspectives in Plant Ecology, Evolution and Systematics, 17, 66-77.

Liu G, Wang L, Jiang L et al. (2018) Specific leaf area predicts dryland litter decomposition via two mechanisms. Journal of Ecology, 106, 218-229.

Liu W, Zheng C, Fu Z et al. (2014) Facilitation of seedling growth and nutrient uptake by indigenous arbuscular mycorrhizal fungi in intensive agroecosytems. Biology and Fertility of Soils, 50, 381-394.

Loreau M, Hector A (2001) Partitioning selection and complementarity in biodiversity experiments. Nature, 412, 72-6.

Loreau M, Naeem S, Inchausti P et al. (2001) Biodiversity Current and and Future Functioning : Challenges Knowledge. Science, 294, 804-808.

Louca S, Polz MF, Mazel F et al. (2018) Function and functional redundancy in microbial systems. Nature Ecology \& Evolution.

Luster J, Göttlein A, Nowack B, Sarret G (2009) Sampling, defining, characterising and modeling the rhizosphere-the soil science tool box. Plant and Soil, 321, 457-482.

Ma Z, Guo D, Xu X et al. (2018) Evolutionary history resolves global organization of root functional traits. Nature, 555, 94-97.

Maherali H (2014) Is there an association between root architecture and mycorrhizal growth response? New Phytologist, 204, 192-200.

Mandyam K, Fox C, Jumpponen A (2012) Septate endophyte colonization and host responses of grasses and forbs native to a tallgrass prairie. Mycorrhiza, 22, 109-119.

Mandyam K, Jumpponen A (2008) Seasonal and temporal dynamics of arbuscular mycorrhizal and dark septate endophytic fungi in a tallgrass prairie ecosystem are minimally affected by nitrogen enrichment. Mycorrhiza, 18, 145-155.

Maron JL, Marler M, Klironomos JN, Cleveland CC (2011) Soil fungal pathogens and the relationship between plant diversity and productivity. Ecology Letters, 14, 36-41.

Marquard E, Schmid B, Roscher C et al. (2013) Changes in the Abundance of Grassland Species in Monocultures versus Mixtures and Their Relation to Biodiversity Effects. PLoS ONE, 8, e75599.

Marschner P, Yang CH, Lieberei R, Crowley DE (2001) Soil and plant specific effects on bacterial community composition in the rhizosphere. Soil Biology \& Biochemistry, 33, 1437-1445.

Martínez-García LB, Richardson SJ, Tylianakis JM, Peltzer D a, Dickie I a (2015) Host identity is a dominant driver of mycorrhizal fungal community composition during ecosystem development. New Phytologist, 205, 1565-1576.

Masella AP, Bartram AK, Truszkowski JM, Brown DG, Neufeld JD (2012) PANDAseq: 
Paired-end assembler for illumina sequences. BMC Bioinformatics, 13, 1-7.

McDonald D, Clemente JC, Kuczynski J et al. (2012) The Biological Observation Matrix (BIOM) format or: how I learned to stop worrying and love the ome-ome. GigaScience, 1, 7.

Meding SM, Zasoski RJ (2008) Hyphal-mediated transfer of nitrate, arsenic, cesium, rubidium, and strontium between arbuscular mycorrhizal forbs and grasses from a California oak woodland. Soil Biology and Biochemistry, 40, 126-134.

Mellado-Vázquez PG, Lange M, Bachmann D et al. (2016) Plant diversity generates enhanced soil microbial access to recently photosynthesized carbon in the rhizosphere. Soil Biology and Biochemistry, 94, 122-132.

De Meyer SE, Van Hoorde K, Vekeman B, Braeckman T, Willems A (2011) Genetic diversity of rhizobia associated with indigenous legumes in different regions of Flanders (Belgium). Soil Biology and Biochemistry, 43, 2384-2396.

Meyer ST, Ebeling A, Eisenhauer N et al. (2016) Effects of biodiversity strengthen over time as ecosystem functioning declines at low and increases at high biodiversity. Ecosphere, 7.

Mikkelsen BL, Rosendahl S, Jakobsen I (2008) Underground resource allocation between individual networks of mycorrhizal fungi. New Phytologist, 180, 890-898.

Milcu A, Partsch S, Langel R, Scheu S (2006) The response of decomposers (earthworms, springtails and microorganisms) to variations in species and functional group diversity of plants. Oikos, 112, 513-524.

Millard P, Singh BK (2010) Does grassland vegetation drive soil microbial diversity? Nutrient Cycling in Agroecosystems, 88, 147-158.

Millennium Ecosystem Assessment (2005) Ecosystems and Human Well-being: Synthesis. Island Press, Washington, DC.

Moll J, Hoppe B, König S et al. (2016) Spatial Distribution of Fungal Communities in an Arable Soil. PLoS ONE, 11, 1-19.

Mommer L, Cotton TEA, Raaijmakers JM et al. (2018) Lost in diversity: the interactions between soil-borne fungi, biodiversity and plant productivity. New Phytologist, 218, 542-553.

Moora M, Zobel M (1996) Effect of Arbuscular Mycorrhiza on Inter- and Intraspecific Competition of Two Grassland Species. Ecology, 108, 79-84.

Moore JC, Berlow EL, Coleman DC et al. (2004) Detritus, trophic dynamics and biodiversity. Ecology Letters, 7, 584-600.

Mora-Romero GA, Cervantes-Gámez RG, Galindo-Flores H et al. (2015) Mycorrhizainduced protection against pathogens is both genotype-specific and grafttransmissible. Symbiosis, 66, 55-64.

Moreau D, Pivato B, Bru D et al. (2015) Plant traits related to nitrogen uptake influence plantmicrobe competition. Ecology, 96, 2300-2310.

Morriën E, Hannula SE, Snoek LB et al. (2017) Soil networks become more connected and take up more carbon as nature restoration progresses. Nature Communications, $\mathbf{8}$, 14349.

Naeem S, Thompson LJ, Lawler SP, Lawton JH, Woodfin RM (1994) Declining biodiversity can alter the performance of ecosystems. Nature, 368, 734-737.

Nelson MB, Martiny AC, Martiny JBH (2016) Global biogeography of microbial nitrogencycling traits in soil. Proceedings of the National Academy of Sciences, 113, 80338040.

Nguyen NH, Song Z, Bates ST et al. (2016) FUNGuild: An open annotation tool for parsing fungal community datasets by ecological guild. Fungal Ecology, 20, 241- 


\section{8.}

O’Brien SL, Gibbons SM, Owens SM et al. (2016) Spatial scale drives patterns in soil bacterial diversity. Environmental microbiology, 18, 2039-2051.

Offre P, Spang A, Schleper C (2013) Archaea in biogeochemical cycles. Annual Review of Microbiology, 67, 437-57.

Oksanen AJ, Blanchet FG, Kindt R et al. (2015) Community Ecology Package.

Oostenbrink M (1960) Estimating nematode populations by some selected methods. Nematology, 6, 85-102.

Öpik M, Moora M, Zobel M et al. (2008) High diversity of arbuscular mycorrhizal fungi in a boreal herb-rich coniferous forest. New Phytologist, 179, 867-876.

Oram NJ, Ravenek JM, Barry KE et al. (2018) Below-ground complementarity effects in a grassland biodiversity experiment are related to deep-rooting species. Journal of Ecology, 106, 265-277.

Orwin KH, Buckland SM, Johnson D et al. (2010) Linkages of plant traits to soil properties and the functioning of temperate grassland. Journal of Ecology, 98, 1074-1083.

Osanai Y, Bougoure DS, Hayden HL, Hovenden MJ (2013) Co-occurring grass species differ in their associated microbial community composition in a temperate native grassland. Plant and Soil, 368, 419-431.

Palmer KM, Young JPW (2000) Higher diversity of Rhizobium leguminosarum biovar viciae populations in arable soils than in grass soils. Applied and Environmental Microbiology, 66, 2445-2450.

Pangesti N, Pineda A, Pieterse CMJ, Dicke M, van Loon JJA (2013) Two-way plant mediated interactions between root-associated microbes and insects: from ecology to mechanisms. Frontiers in Plant Science, 4, 1-11.

Philippot L, Raaijmakers JM, Lemanceau P, van der Putten WH (2013) Going back to the roots: the microbial ecology of the rhizosphere. Nature Reviews Microbiology, 11, 789-799.

Philippot L, Spor A, Hénault C et al. (2013) Loss in microbial diversity affects nitrogen cycling in soil. The ISME journal, 7, 1609-19.

Pietikäinen A, Kytöviita MM (2007) Defoliation changes mycorrhizal benefit and competitive interactions between seedlings and adult plants. Journal of Ecology, 95, 639-647.

Pinheiro J, Bates D, DebRoy S, Sarkar D, Team TRDC (2015) nlme: Linear and Nonlinear Mixed Effects Models.

Poorter H, Niklas KJ, Reich PB et al. (2011) Biomass allocation to leaves, stems and roots: meta-analysis of interspecific variation and environmental control. New Phytologist, 193, 30-50.

Porazinska DL, Bardgett RD, Blaauw MB et al. (2003) Relationships at the AbovegroundBelowground Interface: Plants, Soil Biota, and Soil Processes. , 73, 397-399.

Priebe S, Kreisel C, Horn F, Guthke R, Linde J (2015) FungiFun2: A comprehensive online resource for systematic analysis of gene lists from fungal species. Bioinformatics, 31, 445-446.

Prober SM, Leff JW, Bates ST et al. (2015) Plant diversity predicts beta but not alpha diversity of soil microbes across grasslands worldwide. Ecology Letters, 18, 85-95.

Prosser JI (2012) Ecosystem processes and interactions in a morass of diversity. FEMS Microbiology Ecology, 81, 507-519.

Pruesse E, Peplies J, Glöckner FO (2012) SINA: accurate high-throughput multiple sequence alignment of ribosomal RNA genes. Bioinformatics (Oxford, England), 28, $1823-9$. 
Van der Putten WH, Bardgett RD, Bever JD et al. (2013) Plant-soil feedbacks: The past, the present and future challenges. Journal of Ecology, 101, 265-276.

Quast C, Pruesse E, Yilmaz P et al. (2013) The SILVA ribosomal RNA gene database project: improved data processing and web-based tools. Nucleic acids research, $\mathbf{4 1}$, D590-6.

Quince C, Lanzen A, Davenport RJ, Turnbaugh PJ (2011) Removing Noise From Pyrosequenced Amplicons. BMC Bioinformatics, 12, 38.

Quist CW, Schrama M, de Haan JJ et al. (2016) Organic farming practices result in compositional shifts in nematode communities that exceed crop-related changes. Applied Soil Ecology, 98, 254-260.

Ramirez KS, Leff JW, Barberán A et al. (2014) Biogeographic patterns in below-ground diversity in New York City's Central Park are similar to those observed globally. Proceedings of the Royal Society B: Biological Sciences, 281, 1-9.

Ravenek JM, Bessler H, Engels C et al. (2014) Long-term study of root biomass in a biodiversity experiment reveals shifts in diversity effects over time. Oikos, 123, 1528-1536.

Read DJ, Perez-Moreno J (2003) Mycorrhizas and Nutrient Cycling in Ecosystems: A Journey towards Relevance? New Phytologist, 157, 475-492.

Reich PB (2014) The world-wide 'fast-slow' plant economics spectrum: a traits manifesto. Journal of Ecology, 102, 275-301.

Ritz K, Young IM (2004) Interactions between soil structure and fungi. Mycologist, 18, 5259.

Rodríguez-Echeverría S, Moreno S, Bedmar EJ (2014) Genetic diversity of root nodulating bacteria associated with Retama sphaerocarpa in sites with different soil and environmental conditions. Systematic and Applied Microbiology, 37, 305-310.

Rognes T, Flouri T, Nichols B, Quince C, Mahé F (2016) VSEARCH: a versatile open source tool for metagenomics. PeerJ, 4, e2584.

Roscher C, Schumacher J, Baade J (2004) The role of biodiversity for element cycling and trophic interactions: an experimental approach in a grassland community. Basic and Applied Ecology, 121, 107-121.

Roscher C, Schumacher J, Gubsch M et al. (2018) Origin context of trait data matters for predictions of community performance in a grassland biodiversity experiment. Ecology, 0, 1-13.

Roscher C, Thein S, Weigelt A et al. (2011) N2 fixation and performance of 12 legume species in a 6-year grassland biodiversity experiment. Plant and Soil, 341, 333-348.

Rousk J, Bååth E, Brookes PC et al. (2010) Soil bacterial and fungal communities across a $\mathrm{pH}$ gradient in an arable soil. Isme Journal, 4, 1340-1351.

Van Ruijven J, Berendse F (2009) Long-term persistence of a positive plant diversityproductivity relationship in the absence of legumes. Oikos, 118, 101-106.

Ruotsalainen AL, Väre H, Vestberg M (2002) Seasonality of root fungal colonization in low-alpine herbs. Mycorrhiza, 12, 29-36.

Saks Ü, Davison J, Öpik M et al. (2014) Root-colonizing and soil-borne communities of arbuscular mycorrhizal fungi in a temperate forest understorey 1. Botany, 285, 277285.

Sala OE, Chapin FS, Armesto JJ et al. (2000) Global biodiversity scenarios for the year 2100. Science, 287, 1770-1774.

Sanaullah M, Chabbi A, Maron PA et al. (2016) How do microbial communities in top- and subsoil respond to root litter addition under field conditions? Soil Biology and Biochemistry, 103, 28-38. 
Sasse J, Martinoia E, Northen T (2018) Feed Your Friends: Do Plant Exudates Shape the Root Microbiome? Trends in Plant Science, 23, 25-41.

Sauheitl L, Glaser B, Dippold M, Leiber K, Weigelt A (2010) Amino acid fingerprint of a grassland soil reflects changes in plant species richness. Plant and Soil, 334, 353363.

Sayer EJ, Oliver AE, Fridley JD et al. (2017) Links between soil microbial communities and plant traits in a species-rich grassland under long-term climate change. Ecology and Evolution, 7, 855-862.

Scherber C, Eisenhauer N, Weisser WW et al. (2010) Bottom-up effects of plant diversity on multitrophic interactions in a biodiversity experiment. Nature, 468, 553-556.

Scherer-Lorenzen M (2008) Functional diversity affects decomposition processes in experimental grasslands. Functional Ecology, 22, 547-555.

Scheublin TR, Ridgway KP, Young JPW, Van Der Heijden MGA (2004) Nonlegumes, legumes, and root nodules harbor different arbuscular mycorrhizal fungal communities. Applied and Environmental Microbiology, 70, 6240-6246.

Schittko C, Runge C, Strupp M, Wolff S, Wurst S (2016) No evidence that plant-soil feedback effects of native and invasive plant species under glasshouse conditions are reflected in the field. Journal of Ecology, 104, 1243-1249.

Schlatter DC, Bakker MG, Bradeen JM, Kinkel LL (2015) Plant community richness and microbial interactions structure bacterial communities in soil. Ecology, 96, 134-142.

Schloss PD, Westcott SL, Ryabin T et al. (2009) Introducing mothur: Open-source, platform-independent, community-supported software for describing and comparing microbial communities. Applied and Environmental Microbiology, 75, 7537-7541.

Schnitzer SA, Klironomos JN, HilleRisLambers J, Kinkel LL, Reich PB (2011) Soil microbes drive the classic plant diversity-productivity pattern. Ecology, 92, 296-303.

Schulz-Bohm K, Martín-Sánchez L, Garbeva P (2017) Microbial volatiles: Small molecules with an important role in intra- and inter-kingdom interactions. Frontiers in Microbiology, 8, 1-10.

Schulz B (2006) Mutualistic Interactions with Fungal Root Endophytes. In: Soil Biology (eds Schulz BJE, Boyle CJC, Sieber TN), p. vol 9. Springer, Berlin, Heidelberg.

Schulz B, Boyle C (2005) The endophytic continuum. Mycological Research, 109, 661686.

Sessitsch A, Weilharter A, Martin H et al. (2001) Microbial Population Structures in Soil Particle Size Fractions of a Long-Term Microbial Population Structures in Soil Particle Size Fractions of a Long-Term Fertilizer Field Experiment. Applied and Environmental Microbiology, 67, 4215-4224.

Setälä H, McLean MA (2004) Decomposition rate of organic substrates in relation to the species diversity of soil saprophytic fungi. Oecologia, 139, 98-107.

Simard SW, Beiler KJ, Bingham MA et al. (2012) Mycorrhizal networks: Mechanisms, ecology and modelling. Fungal Biology Reviews, 26, 39-60.

Simpson EH (1949) Measurement of Diversity. Nature, 163, 688-688.

Smith-Ramesh LM, Reynolds HL (2017) The next frontier of plant-soil feedback research: unraveling context dependence across biotic and abiotic gradients. Journal of Vegetation Science, 28, 484-494.

Smith SE, Read DJ (2010) Mycorrhizal Symbiosis. Academic Press, London, UK.

Soliveres S, Manning P, Prati D et al. (2016) Locally rare species influence grassland ecosystem multifunctionality Locally rare species influence grassland ecosystem multifunctionality.

Spehn EM, Jasmin J, Schmid B, Alphei J, Körner C (2000) Plant diversity effects on soil 
heterotrophic activity in experimental grassland ecosystems. Plant and Soil, 224, 217-230.

Spehn EM, Scherer-Lorenzen M, Schmid B et al. (2002) The role of legumes as a component of biodiversity in a cross-European study of grassland biomass nitrogen. Oikos, 98, 205-218.

Steinauer K, Fischer FM, Roscher C, Scheu S, Eisenhauer N (2017) Spatial plant resource acquisition traits explain plant community effects on soil microbial properties. Pedobiologia, 65, 50-57.

Steinbeiss S, Beßler H, Engels C et al. (2008) Plant diversity positively affects short-term soil carbon storage in experimental grasslands. Global Change Biology, 14, 2937 2949.

Steinkellner S, Lendzemo V, Langer I et al. (2007) Flavonoids and strigolactones in root exudates as signals in symbiotic and pathogenic plant-fungus interactions. Molecules, 12, 1290-1306.

Strecker T, Barnard RL, Niklaus PA et al. (2015) Effects of Plant Diversity, Functional Group Composition, and Fertilization on Soil Microbial Properties in Experimental Grassland. Plos One, 10, e0125678.

Sugiyama S, Zabed HM, Okubo A (2008) Relationships between soil microbial diversity and plant community structure in seminatural grasslands. Grassland Science, 54, $117-124$.

Sun R, Dsouza M, Gilbert JA et al. (2016) Fungal community composition in soils subjected to long-term chemical fertilization is most influenced by the type of organic matter. Environmental Microbiology, 18, 5137-5150.

Tedersoo L, Bahram M, Cajthaml T et al. (2015) Tree diversity and species identity effects on soil fungi, protists and animals are context dependent. The ISME Journal, 1-17.

Teste FP, Simard SW, Durall DM, Guy RD, Berch SM (2010) Net carbon transfer between Pseudotsuga menziesii var. Glauca seedlings in the field is influenced by soil disturbance. Journal of Ecology, 98, 429-439.

Tiedje JM, Cho JC, Murray A et al. (2001) Soil Teeming with Life : New Frontiers for Soil Science. In: Sustainable Management of Soil Organic Matter (eds Rees RM, Ball BC, Campbell CD, Watson CA), pp. 393-412. Wallingford, U.K.

Tilman D, Reich PB, Knops J et al. (2001) Diversity and productivity in a long-term grassland experiment. Science, 294, 843-845.

Tilman D, Wedin D, Knops J (1996) Productivity and sustainability influenced by biodiversity in grassland ecosystems. Nature, 379, 718-720.

Torsvik V, Øvreås L (2002) Microbial diversity and function in soil: from genes to ecosystems. Curr Opin Microbiol, 5, 240-245.

Turner TR, Ramakrishnan K, Walshaw J et al. (2013) Comparative metatranscriptomics reveals kingdom level changes in the rhizosphere microbiome of plants. The ISME journal, 7, 2248-58.

Ulrich A, Becker R (2006) Soil parent material is a key determinant of the bacterial community structure in arable soils. FEMS Microbiology Ecology, 56, 430-443.

Valverde-Barrantes OJ, Smemo KA, Feinstein LM, Kershner MW, Blackwood CB (2013) The distribution of below-ground traits is explained by intrinsic species differences and intraspecific plasticity in response to root neighbours. Journal of Ecology, 101, 933-942.

Vályi K, Rillig MC, Hempel S (2015) Land-use intensity and host plant identity interactively shape communities of arbuscular mycorrhizal fungi in roots of grassland plants. The New phytologist, 205, 1577-86. 
Vandenkoornhuyse P, Baldauf SL, Leyval C, Straczek J, Young JPW (2002) Extensive fungal diversity in plant roots. Science, 295, 2051.

Vandenkoornhuyse P, Husband R, Daniell TJ. et al. (2002) Arbuscular mycorrhizal community composition associated with two plant species in a grassland ecosystem_2002_Molecular Ecology.pdf. , 1555-1564.

Venables WN, Ripley BD (2002) Modern applied statistics with S. Springer, New York.

Veresoglou SD, Chen B, Rillig MC (2012) Arbuscular mycorrhiza and soil nitrogen cycling. Soil Biology and Biochemistry, 46, 53-62.

Vierheilig H, Coughlan AP, Wyss URS, Recherche C De (1998) Ink and Vinegar, a Simple Staining Technique for Arbuscular-Mycorrhizal Fungi. Applied and Environmental Microbiology, 64, 5004-5007.

Vierheilig H, Lerat S, Piché Y (2003) Systemic inhibition of arbuscular mycorrhiza development by root exudates of cucumber plants colonized by Glomus mosseae. Mycorrhiza, 13, 167-170.

van de Voorde TFJ, van der Putten WH, Martijn Bezemer T (2011) Intra- and interspecific plant-soil interactions, soil legacies and priority effects during old-field succession. Journal of Ecology, 99, 945-953.

Vos M, Wolf AB, Jennings SJ, Kowalchuk GA (2013) Micro-scale determinants of bacterial diversity in soil. FEMS Microbiology Reviews, 37, 936-954.

de Vries FT, Manning P, Tallowin JRB et al. (2012) Abiotic drivers and plant traits explain landscape-scale patterns in soil microbial communities. Ecology Letters, 15, 12301239.

Wagg C, Bender SF, Widmer F, van der Heijden MGA (2014) Soil biodiversity and soil community composition determine ecosystem multifunctionality. Proceedings of the National Academy of Sciences, 111, 5266-5270.

Wagg C, Ebeling A, Roscher C et al. (2017) Functional trait dissimilarity drives both species complementarity and competitive disparity. Functional Ecology, 31, 23202329.

Wagg C, Jansa J, Stadler M, Schmid B, Van Der Heijden MGA (2011) Mycorrhizal fungal identity and diversity relaxes plant-plant competition. Ecology, 92, 1303-1313.

Walder F, Niemann H, Natarajan M et al. (2012) Mycorrhizal Networks: Common Goods of Plants Shared under Unequal Terms of Trade. Plant Physiology, 159, 789-797.

Waldrop MP, Zak DR, Blackwood CB, Curtis CD, Tilman D (2006) Resource availability controls fungal diversity across a plant diversity gradient. Ecology Letters, 9, 11271135 .

Wang Q, Garrity GM, Tiedje JM, Cole JR (2007) Naive Bayesian classifier for rapid assignment of rRNA sequences into the new bacterial taxonomy. Applied and Environmental Microbiology, 73, 5261-5267.

Wardle D (2002) Communities and Ecosystems: Linking the Aboveground and Belowground Components. Princeton University Press.

Wardle D a. (2004) Ecological Linkages Between Aboveground and Belowground Biota. Science, 304, 1629-1633.

Wardle DA, Yeates GW, Williamson W, Bonner KI (2003) The Response of a Three Trophic Level Soil Food Web to the Identity and Diversity of Plant Species and Functional Groups. Oikos, 102, 45-56.

Wearn JA, Sutton BC, Morley NJ, Gange AC (2012) Species and organ specificity of fungal endophytes in herbaceous grassland plants. Journal of Ecology, 100, 1085 1092.

Wehner J, Antunes PM, Powell JR, Caruso T, Rillig MC (2011) Indigenous Arbuscular 
Mycorrhizal Fungal Assemblages Protect Grassland Host Plants from Pathogens. PLoS ONE, 6, e27381.

Wehner J, Powell JR, Muller LAH et al. (2014) Determinants of root-associated fungal communities within Asteraceae in a semi-arid grassland. Journal of Ecology, 102, 425-436.

Weishampel PA, Bedford BL (2006) Wetland dicots and monocots differ in colonization by arbuscular mycorrhizal fungi and dark septate endophytes. Mycorrhiza, 16, 495-502.

Weremijewicz J, Janos DP (2013) Common mycorrhizal networks amplify size inequality in Andropogon gerardii monocultures. New Phytologist, 198, 203-213.

Weremijewicz J, Sternberg L da SL, Janos DP (2016) Common mycorrhizal networks amplify competition by preferential mineral nutrient allocation to large host plants. New Phytologist, 212, 461-471.

Whittaker RH (1972) Evolution and Measurement of Species Diversity. , 21, 213-251.

Wickham H (2009) Elegant graphics for data analysis. Springer-Verlag New York.

Wilson GW, Hartnett DC (1998) Interspecific variation in plant responses to mycorrhizal colonization in tallgrass prairie. American journal of botany, 85, 1732-1738.

Wintzingerode F, Göbel UB, Stackebrandt E (1997) Determination if microbial diversity in environmental samples: pitfalls of PCR-based analysis. FEMS Microbiol. Rev., 21, 213-229.

Wright AJ, de Kroon H, Visser EJW et al. (2017) Plants are less negatively affected by flooding when growing in species-rich plant communities. New Phytologist, 213, 645-656.

Xiong W, Jousset A, Guo S et al. (2018) Soil protist communities form a dynamic hub in the soil microbiome. ISME Journal, 12, 634-638.

Yang T, Adams JM, Shi Y et al. (2017) Soil fungal diversity in natural grasslands of the Tibetan Plateau: associations with plant diversity and productivity. New Phytologist, 215, 756-765.

Yeates GW, Bongers T, De Goede RG, Freckman DW, Georgieva SS (1993) Feeding habits in soil nematode families and genera-an outline for soil ecologists. Journal of nematology, 25, 315-331.

Zak DR, Holmes WE, White DC, Peacock AD, Tilman D (2003) Plant Diversity, Soil Microbial Communities, and Ecosystem Function: Are There Any Links? Ecology, 84, 2042-2050.

Zhalnina K, Dias R, de Quadros PD et al. (2015) Soil pH Determines Microbial Diversity and Composition in the Park Grass Experiment. Microbial Ecology, 69, 395-406.

Zhang C, Liu G, Xue S, Wang G (2015) Changes in rhizospheric microbial community structure and function during the natural recovery of abandoned cropland on the Loess Plateau, China. Ecological Engineering, 75, 161-171.

Zhang T, Sun Y, Song Y, Tian C, Feng G (2011) On-site growth response of a desert ephemeral plant, Plantago minuta, to indigenous arbuscular mycorrhizal fungi in a central Asia desert. Symbiosis, 55, 77-84. 
Summary/Samenvatting 
Soil biota are critical to terrestrial ecosystem functioning. Plant and soil communities are engaged in dynamic interactions, however, plant species richness is declining worldwide and little is known about what this means for soil biodiversity. To date, many studies have focussed on the effects of soil biota on plant performance and plant community dynamics, while the consequences of declining plant species diversity on richness and abundance of soil biota is not yet well understood. In this thesis I aim to increase mechanistic understanding of the impact of plant species richness and community composition on the richness and abundance of soil microbes and nematodes. I investigated (a) the relative impact of plant community changes (plant species richness and functional group identity) on soil communities compared to abiotic factors, (b) which plant community changes (plant species richness, functional group identity, specific traits, and functional diversity) affect microbial communities in roots and soil most, (c) which microbial groups of the soil food web are most sensitive to changes in plant community composition, and (d) how connections with mycorrhizal networks affect plant performance.

I performed my study in the Jena plant species and trait diversity experiments (Germany) and in a chronosequence of ex-arable fields in the Veluwe area of the Netherlands. In the Jena plant diversity experiment, I show that soil abiotic properties within the same field alter community composition of bacteria, mycorrhizal and non-mycorrhizal fungi, protists, and archaea more strongly than plant species richness or plant functional group identity (Chapter 2). Among the abiotic factors measured, soil texture (the proportions of sand, silt, and clay) explained most of the variation in community composition of all the microbial groups analysed. In the Jena Experiment the $\mathrm{pH}$ gradient is relatively narrow. I show that such minor variation near neutral soil $\mathrm{pH}$ does not have a substantial impact on microbial community composition.

Despite the considerable impact of abiotic soil properties my results show that multiple aspects of plant species and community diversity affect microbial and nematode community composition, and that plant traits have stronger effects than plant species diversity. I found that the richness of fungi in the bulk soil, root-associated fungi and root-associated fungal saprophytes increased with plant species richness. However, I found no such response of fungal plant pathogens, arbuscular mycorrhizal fungi (AMF), bacteria, protists, or nematodes with respect to plant species richness (Chapters 2, 3, 4). In contrast, plant functional group identity altered richness and abundance of soil biota with marked difference between plant communities with and without legumes (Chapter 2). The 


\section{Summary}

community composition of fungi in soil and roots, as well as of nematodes in soil correlated with the percentage cover of grasses and forbs (Chapters 3, 4).

The community weighted mean of specific morphology and development-related plant traits explained more variation in soil community composition than plant functional group or plant species richness (Chapters 3, 4). However, functional diversity (quantified as variation in plant morphological and development traits), had limited effects on community composition of soil biota. Bacterial richness was positively related to functional diversity, but there were no such effect on the richness or abundance of nematodes, or of soil- and root-associated fungi (Chapters 3, 4). For the group of soil biota examined, I show that compositional changes in the plant community have the strongest effect on the richness and abundance on fungal communities, a smaller effect on nematode communities, and the least effect on bacterial communities (Chapters 2, 4).

I examined how plant traits alter the richness and community composition of bacteria, fungi and nematodes from the green (living plant-based) and brown (dead plant-based) pathways in the soil food web. These pathways reflect the energy channels in which organic matter flows from the primary producer (the plant) to consumer groups. Differences in plant trait composition affect the abundance of soil biota from the green pathway of the soil food web more than soil biota from the brown pathway of the soil food web. I also analysed whether plant traits and diversity effects differ between lower and higher trophic positions in the soil food web. I found that plant traits and diversity effects have the strongest impact on the abundance of lowest trophic position organisms in the soil food web (Chapter 4).

I tested the functional consequences of plant seedlings being linked to the mycorrhizal network in soil. In contrast to the original expectations, my results show that established mycorrhizal networks did not promote the performance of seedlings. Instead, I found biomass reduction of seedlings that were connected to a mycorrhizal network and this was independent of successional stage, plant functional group identity and previously reported negative or positive plant-soil feedback interactions. In addition, increased levels of AMF colonisation correlated with decreased seedling performance (Chapter 5). Apparently, seedling establishment in the field is hampered, as opposed to promoted by AMF networks in restored grasslands. 
I conclude that:

- In a long-term grassland biodiversity experiment, increasing plant species richness leads to higher root-associated and bulk soil fungal species richness but has no significant impact on the richness of AMF, bacteria, protists, or archaea in soil.

- Soil microbial community composition in bulk soil can be influenced more by changes in abiotic soil properties and plant functional group identity, than by changes in plant species richness per se.

- Soil and root-associated fungal richness and community composition is influenced more by plant structural and development traits than by plant species richness.

- Plant species richness does not affect richness and abundance of symbionts (AMF), fungal plant pathogens and root feeding nematodes in soil. Instead, these functional groups of soil biota that are associated with positive and negative plantsoil feedback are altered by changes in plant trait composition.

- Plant trait composition affects the abundance of soil biota from the green pathway (living plant-based) in the soil food web more than from the brown pathway (dead plant-based) of the soil food web.

- Differences in plant trait composition have the strongest impact on the abundance of lowest trophic position organisms in the soil food web.

- Plant functional diversity (quantified as variation in plant morphological and development traits) does not seem to be a significant determinant of rootassociated fungi, fungi in soil and nematode community composition or richness.

- In secondary succession grasslands, mycorrhizal networks reduce the biomass of seedlings, independent of successional stage, plant functional group identity, and negative or positive plant-soil feedback interactions.

- Biomass reduction in seedlings connected to a mycorrhizal network is associated with increased levels of AMF colonisation. 


\section{Samenvatting}

\section{Samenvatting}

Het bodemleven is van groot belang voor het functioneren van terrestrische ecosystemen doordat plant- en bodemgemeenschappen verwikkeld zijn in dynamische interacties. Echter, plantensoortenrijkdom neemt wereldwijd af en er is weinig kennis over de gevolgen van deze afname op de diversiteit van het bodemleven. Tot op heden hebben wetenschappelijke studies zich voornamelijk gericht op de effecten van het bodemleven op plantenontwikkeling en plantgemeenschapsdynamiek, terwijl de consequenties van afnemende plantensoortenrijkdom op soortenrijkdom en abundantie van het bodemleven nog niet duidelijk bekend zijn. In dit proefschrift streef ik ernaar om meer inzicht te verkrijgen in de impact van de soortenrijkdom en samenstelling van plantengemeenschappen op de rijkdom en talrijkheid van microben en nematoden in de bodem en in plantenwortels. Ik onderzocht (a) de relatieve invloed van veranderingen in plantengemeenschappen (plantensoortenrijkdom en functionele plantengroepen) op bodemgemeenschappen ten opzichte van abiotische factoren, (b) welke veranderingen in de plantengemeenschap (plantensoortenrijkdom, functionele plantengroepen, specifieke planteigenschappen en functionele diversiteit) de grootste invloed hebben op microbengemeenschappen in de bodem en plantenwortels, (c) welke microbiële- en nematodengroepen in het bodemvoedselweb het meest gevoelig zijn voor veranderingen in de samenstelling van plantengemeenschappen, en (d) hoe verbinding met het netwerk van mycorrhizaschimmels plantengroei beïnvloedt.

De eerste drie onderzoekhoofdstukken van dit proefschrift zijn uitgevoerd in het graslandbiodiversiteitsexperiment in Jena (Duitsland). Voor het eerste onderzoekhoofdstuk maakte ik gebruik van het Jena hoofdexperiment, waarin plantengemeenschappen variëren in soortenrijkdom en aantal functionele plantengroepen (grassen, vlinderbloemigen, kleine en grote kruiden). Voor het tweede en derde onderzoekhoofdstuk makkte ik gebruik van het 'trait-based' experiment, waarin een gradiënt is gerealiseerd met plantengemeenschappen die zeer gelijkaardige tot zeer uiteenlopende eigenschappen hebben. De gebruikte planteneigenschappen zijn maximale worteldiepte, worteldichtheid, planthoogte, bladoppervlak, groeistart en bloeistart.

In het Jena hoofdexperiment liet ik zien dat abiotische bodemfactoren de gemeenschapssamenstelling van bacteriën, mycorrhiza en andere schimmels, protisten en archaea verandert. Daarnaast liet ik zien dat bodem-abiotiek een sterkere invloed heeft op de gemeenschapssamenstelling van micro-organismen dan plantensoortenrijkdom of de 
identiteit van de functionele plantengroepen (Hoofdstuk 2). Van de gemeten abiotische factoren verklaarde bodemtextuur (de proportie zand, silt en klei) de meeste variatie in de microbiële gemeenschapssamenstelling. De pH (zuurgraad) variatie tussen de verschillende veldjes in het Jena experiment is relatief klein. Ik liet zien dat zulke geringe $\mathrm{pH}$ variaties geen substantieel effect hebben op de samenstelling van microbiële gemeenschappen, ook al is bodem $\mathrm{pH}$ op grotere (regionale tot wereldwijde) schaal vaak een factor die sterk relateert aan de samenstelling van het microbiële leven in de bodem.

Ondanks dat bodem-abiotiek een aanzienlijke invloed heeft op de gemeenschapssamenstelling van micro-organismen, laten mijn resultaten ook zien dat verschillende aspecten van plantensoorten en diversiteit in de plantengemeenschap invloed hebben op de samenstelling van microben en nematoden in de bodem. Toenemende plantensoortenrijkdom had een positief effect op het aantal schimmelsoorten in de bodem en in en op plantenwortels. Daarbij nam in plantenwortels ook het aantal saprotrofe schimmels, die van dood organisch materiaal leven, toe in soortenaantal. In tegenstelling tot de totale gemeenschap aan schimmels en saprotrofe schimmels had plantensoortenrijkdom geen effect op de soortenaantallen van plantpathogene schimmels, arbusculaire mycorrhizaschimmels, bacteriën, protisten, archaea of nematoden (hoofdstukken 2, 3, 4). De functionele planten groep met het sterkste effect op de samenstelling van microorganismen in de bodem was de familie van vlinderbloemigen (Hoofdstuk 2). In het 'traitbased' experiment, waarin geen vlinderbloemigen voorkomen, liet ik zien dat ook het bedekkingspercentage van grassen en kruiden samenhing met de gemeenschapssamenstelling van nematoden en schimmels in de bodem en met schimmels in de plantenwortels (Hoofdstukken 3, 4).

Uit het 'trait-based' experiment bleek dat diversiteit in planteneigenschappen binnen een plantengemeenschap vrijwel geen invloed heeft op de soortenrijkdom en gemeenschapssamenstelling van microben en nematoden in de bodem of in de wortels. Alleen de soortenrijkdom van bodembacteriën nam toe met toenemende diversiteit in planteneigenschappen, terwijl alle andere microbiële- en nematodengroepen niet zichtbaar reageerden op toenemende variatie in planteneigenschappen. Wel bleek dat veranderingen van specifieke eigenschappen van invloed waren op het bodemleven en dat het bodemleven sterker beïnvloed wordt door specifieke planteneigenschappen dan door plantensoortenrijkdom, de identiteit van de functionele plantengroepen, of de mate van variatie in planteneigenschappen (Hoofdstukken 3, 4). Uit mijn resultaten komt ook naar voren dat voornamelijk schimmelgemeenschappen reageren op veranderingen van 


\section{Samenvatting}

planteigenschappen, terwijl die eigenschappen in mindere mate effect hebben op nematoden en het minste effect hebben op bacteriegemeenschappen (Hoofdstukken 2, 4).

Micro-organismen en nematoden vervullen een belangrijke rol in het bodemvoedselweb. Deze organismen breken dood en levend organisch materiaal af en maken voor planten beschikbare voedingsstoffen vrij. Organismen die zich als pathogeen voeden op levend plantenmateriaal, of in symbiose met planten leven, behoren tot het "groene energiekanaal" van het bodemvoedselweb. Organismen die van dood plantenmateriaal en plantenresiduen leven, behoren tot het "bruine energiekanaal" van het bodemvoedselweb. Ik heb gekeken hoe veranderingen in planteneigenschappen de soortenrijkdom en talrijkheid van organismen in het groene en bruine energiekanaal van het bodemvoedselweb beïnvloeden. Mijn resultaten lieten zien dat veranderingen in planteneigenschappen voornamelijk de talrijkheid van organismen in het groene energiekanaal beïnvloeden. Ook laten de resultaten zien dat veranderingen in planteneigenschappen een groter effect hebben op organismen van lager trofisch (voedsellaag) niveau (bacteriën, schimmel- en plantenetende nematoden) vergeleken met organismen van hoger trofisch niveaus (bacterie- en schimmeletende nematoden, omnivoren en predatoren) (Hoofdstuk 4).

Planten- en bodemgemeenschappen beïnvloeden elkaar wederzijds. Sommige plantensoorten veroorzaken een negatieve terugkoppeling, doordat ze een toename van ziekteverwekkers in de bodem veroorzaken. Dit is de voornaamste reden waarom in de landbouw vruchtwisseling wordt toegepast. Ook zijn er plantensoorten die een positieve terugkoppeling hebben, doordat ze symbiotische micro-organismen, zoals arbusculaire mycorrhizaschimmels, aantrekken. Deze groep schimmels kan een symbiose aangaan met het overgrote deel van alle plantensoorten, hetgeen inhoudt dat de schimmels bodemmineralen, met name fosfaat, en water uitwisselen voor plantensuikers. Uit kasexperimenten is relatief veel bekend over de invloed van deze schimmels op plantengroei. Een eigenschap van deze groep schimmels is dat ze met hun schimmeldraden een netwerk kunnen vormen, waarin meerdere planten, ook van verschillende soorten, met elkaar in verbinding staan. Hoewel kasexperimenten fundamentele kennis opleveren, wordt de complexiteit van natuurlijke systemen er gewoonlijk niet in meegenomen. Het blijkt dat de gevolgen van de symbioses bepaald kunnen worden door het feit of er al dan niet een ontwikkeld mycorrhizaschimmeldradennetwerk in de bodem aanwezig is. Zowel de omringende plantengemeenschap als andere bodemorganismen kunnen via het mycorrhizanetwerk direct of indirect de interactie tussen een plant en een mycorrhizaschimmel beïnvloeden. 
In een veldexperiment heb ik getest wat de consequenties waren voor zaailingen van verschillende plantensoorten om op te groeien met of zonder aansluiting tot een mycorrhizanetwerk. Dit laatste onderzoekhoofdstuk van mijn proefschrift is uitgevoerd in zes verschillende graslanden op de Veluwe. Deze graslanden bevinden zich op voormalige landbouwgronden en zijn op verschillende momenten in de tijd uit productie genomen, waarna ze natuurlijke ontwikkeling tot half-natuurlijk grasland ondergingen. Tegen de verwachtingen in lieten mijn resultaten zien dat verstoring van het mycorrhizanetwerk positief uitpakte voor de biomassa van de zaailingen. Ik liet ook zien dat het niet uitmaakt of het een zaailing van een gras of kruidachtige plant betrof, of de plant bekend staat als een soort met positieve of negatieve terugkoppeling, of dat de zaailing zich ontwikkelde op vrij recent of op langer geleden uit productie genomen landbouwgrond. De resultaten gaven ook aan dat toenemende kolonisatie van mycorrhizaschimmels samenhangt met geringere biomassa van de zaailingen (Hoofdstuk 5). Hiermee toonde ik aan dat onder deze omstandigheden mycorrhizanetwerken de groei van zaailingen in graslanden blijken te remmen, in plaats van bevorderen.

Ik concludeer dat:

- Microbiële bodemgemeenschappen worden sterker beïnvloed door veranderingen in bodem-abiotiek en functionele plantengroep identiteit dan door plantensoortenrijkdom per se.

- Toenemende plantensoortenrijkdom in grasland leidt tot grotere soortenaantallen van bodem- en wortelschimmels, maar heeft geen aantoonbaar effect op de soortenaantallen van plantpathogene schimmels, arbusculaire mycorrhizaschimmels, bacteriën, protisten, archaea of nematoden.

- Soortenrijkdom en compositie van schimmels in de bodem en wortels worden sterker beïnvloed door veranderingen in specifieke planteigenschappen dan door plantensoortenrijkdom per se.

- Mycorrhizaschimmels, plantpathogene schimmels en plant-etende nematoden, organismen die geassocieerd worden met positieve en negatieve plant-bodem terugkoppeling, worden beïnvloed door veranderingen van specifieke planteneigenschappen en niet door plantensoortenrijkdom per se.

- Veranderingen in planteneigenschappen hebben een sterkere invloed op organismen in het groene energiekanaal van het bodemvoedselweb (consumeren van levende planten) dan in het bruine energiekanaal (consumeren van dode planten en plantresiduen). 
- Veranderingen in planteneigenschappen hebben een sterkere invloed op de talrijkheid van organismen met een lage dan hogere trofische (voedsellaag) positie.

- Diversiteit van planteneigenschappen in een plantengemeenschap lijkt geen goede indicator te zijn voor de soortenrijkdom en gemeenschapssamenstelling van nematoden, bodem- en wortelschimmels.

- In half-natuurlijke graslanden, die zich hebben ontwikkeld op voormalige landbouwgronden, reduceren mycorrhizanetwerken de biomassa van zaailingen, ongeacht de tijd die verstreken is sinds het veld uit productie is genomen, de identiteit van de functionele plantengroep, en of de plant een positieve dan wel negatieve terugkoppeling geeft met de bodem als geheel.

- Toenemende kolonisatie van mycorrhizaschimmels hangt samen met de biomassaafname van zaailingen in een mycorrhizanetwerk. 

Saemenvatting in et Stellingwarfs 
Et bojemleven is ommeraek van belang veur et warken van laandekosystemen. Plaante- en bojemgemienschoppen warken maankmeneer in levendige henne-weer-dennes, mar de plaantesoorteriekdom wodt over de hiele wereld minder en d'r is weinig weet van de gevolgen veur de verscheidenhied in et bojemleven. An now toe hebben wetenschoppelike studies heur veurnaemelik richt op 'e effekten van et bojemleven op plaanteontwikkeling en verspreiding van plaantesoorten in de plaantegemienschop, wiels de gevolgen van ofnemende plaantesoorteriekdom op soorteriekdom en anwezighiedsgraod van bojemleven nog niet dudelik is. In dit proefschrift perbere ik meer dudelikhied te kriegen over de uutwarking van plaantesoorteriekdom en gemienschopssaemenstelling op riekdom en anwezighiedsgraod van bojemmikroben en aolties.

Mikro-orgenismen en aolties doen belangriek wark in et bojemeteri'jeweb. Die orgenismes breken dood en levend organisch materiaol of en maeken veur plaanten beschikbere voedingsstoffen vri'j. Plaante- en bojemgemienschoppen warken van weerskaanten op mekeer in. Pattie plaantesoorten geven verkeerd spul weeromme, deur die plaantesoorten kommen d'r meer ziekteverwekkers in de bojem en dat is de veurnaemste reden in de laandbouw veur wisselbouw. D'r bin ok plaantesoorten die een geunstige warking weerommegeven; een groep orgenismen die d'r bi'j betrokken is bin mycorrhizaschimmels. Die groep schimmels kan tegere leven gaon mit et grootste pat van de plaantesoorten, de schimmel geft bojemmineraolen of veur plaantesukers. 
De onderzukings in dit proefschrift bin veur et grootste pat daon in et experiment veur gröslaand-bioverscheidenhied in Jena (Duutslaand). Op dat proefveld bin velties inzi'jd die verschillend binnen in tallen plaantesoorten, d'r bin velties mit 1, 2, 4, 8, 16 en 60 plaantesoorten, van grössen liekewel as van kruden. Ok bin op dit proefveld plaantegemienschoppen maekt mit eigenschoppen die hiel geliek van aord binnen en mit slim uut mekeer lopende eigenschoppen. Plaanteeigenschoppen die d'r veur bruukt binnen weren de grootste wotteldiepte, wotteldichthied, plaantehoogte, bladoppervlak, gruuistart en bluuistart. Et laeste onderzuuksheufdstok van dit proefschrift is uutvoerd op gröslanen op 'e Veluwe. Ik maekte gebruuk van zes eerdere laandbouwgronden die op ongeliekese mementen uut de perduktie neumen binnen.

Om inzicht te kriegen in wat veur mikro-orgenismen en aolties in de bojem zatten nam ik grondmoonsters. Veur et gadderen van aolties wodden grondmoonsters wusken mit waeter. Aolties bin lichter as gronddielties en deur spoelen, bezinken en zeven kuj' ze scheiden van de grond. Aolties bin goed te zien onder een mikroskoop en zo kan soorte bi'j soorte brocht wodden. Mikro-orgenismen wo'n weerkend uut DNA dat we uut de bojem ofscheided hebben. Hoe dat percies in zien wark gong is lastig om hier kot uut te duden, mar ie kun d'r wel van uutgaon dat et identificeren oons goed lokt is.

Mien risseltaoten laoten zien dat de bojemtextuur (de verholing tussen zaand, silt en klei) de gemienschopssaemenstelling veraandert van mikro-orgenismes. Daornaost leut ik zien dat dit niet-levendige diel van de bojem meer invloed het op de gemienschops-saemenstelling van mikro-orgenismes as plaantesoorteriekdom of de aord van 
plaantefunktionele groepen (grössen, vlinderbloemigen, kleine en grote kruden). De variaosie in de $\mathrm{pH}$ (zoertegraod) tussen de verschillende velties in et Jena-experiment is naor verholing klein. Ik hebbe zien laoten dat zokke kleine $\mathrm{pH}$-variaosies gien wezenlike uutwarking hebben op 'e saemenstelling van mikrolevengemienschoppen.

Ok al het et niet-levendige pat beheurlik invloed, mien risseltaoten laoten ok zien dat verschillende kaanten van plaantesoorten en verscheidenhied in de plaantegemienschop invloed hebben op 'e saemenstelling van mikroben en aolties in de bojem. Een wassende plaanteriekdom gaf een geunstig effekt op et tal schimmelsoorten in de bojem en in en op plaantewottels. Daorbi'j nam in plaantewottels ok et tal saprofyte schimmels, schimmels die van dood organisch materiaol leven, toe in tal soorten, mar d'r was gien effekt op plaanteziekteverwekkende schimmels, arbusculaire mycorrhizaschimmels, bakteries, protisten, oerbakteries of aolties. De plaantefunktionele groep mit et starkste effekt op 'e saemenstelling van mikro-orgenismes in de bojem was de femilie van vlinderbloemigen. Daornaost he'k zien laoten dat as d'r gien vlinderbloemigen binnen, dat dan ok et bedekkingspercentage van grössen en kruden invloed hebben kan op de gemienschopssaemenstelling van aolties en schimmels in de bojem en op schimmels in plaantewottels. Uut veerder onderzuuk dee blieken dat veraanderings van eigenlike plaante-eigenschoppen van invloed weren op et bojemleven. Een belangrieke anwiezer veur de schimmel- en aoltiessaemenstelling was de wotteldichthied van een plaantegemienschop. Ok vun ik dat et bojemleven starker beïnvloeded 
wodt deur eigenlike plaante-eigenschoppen as deur plaantesoorteriekdom, de eigen aord van plaantefunktionele groepen, of de maote van variaosie in plaante-eigenschoppen.

Mikro-organismes en aolties speulen een veurnaeme rolle in et bojemeteri'jeweb. Die orgenismes breken dood en levend organisch materiaol of en maeken veur plaanten beschikbere voedingsstoffen vri'j. Orgenismes die op levend plaantemateriaol teren of in de maande mit plaanten leven heuren bi'j de grune gang van et bojemeteri'jeweb, orgenismes die van dood plaantemateriaol en plaanteoverbliefsels leven heuren bi'j de brune gang van dat web. Ik hebbe keken hoe veraanderings in plaante-eigenschoppen op de soorteriekdom en maote van veurkommen van orgenismes in de grune en brune gang van et bojemeteri'jeweb warken. Mien risseltaoten leuten zien dat veraanderings in plaante-eigenschoppen veural invloed hebben op 'e maote van veurkommen van orgenismes in et grune trajekt. De risseltaoten tonen ok dat veraanderings in plaanteeigenschoppen een groter effekt hebben op orgenismes van leger eteri'jewebnivo (bakteries, schimmels en plaante-etende aolties) vergeleken mit orgenismes van hoger vormingsnivo (bakteries- en schimmeletende aolties, allesetende aolties en roofaolties).

Van kasproefnemings uut is d'r naor verholing veul bekend over de invloed van die mycorrhizaschimmels op plaantegruui. Een eigenschop van die groep schimmels is dat ze mit heur schimmeldraoden een netwark maeken kunnen; verscheiden plaanten, ok van verschillende soorten, kun daor dan mit mekeer in verbunnen wezen. Alhoewel kasproefnemings follementekennis opleveren, kommen ze netuurlik niet toe an de ingewikkeldhied van netuurlike 
systemen. De plaantegemienschop ommetoe liekewel as ere bojemorgenismes kun de henne-weer-denne-warking tussen een plaante en een mycorrhizaschimmel beïnvloeden.

In een veldproefneming hebbe ik uutperbeerd wat de konsekweensies weren veur zi'jplaanten om op te gruuien mit of zonder ansluting op een mycorrhizanetwark. Dit laeste onderzuuksheufdstok van dit proefschrift is uutvoerd op gröslanen op 'e Veluwe. Ik hebbe gebruuk maekt van een zestal eerdere laandbouwgronden die op ongeliekese tiedstippen uut de perduktie neumen binnen. Eers as dat verwaacht wodde, leuten mien risseltaoten zien dat versteuring van et mycorrhizanetwark geunstig uutpakte veur de biomassa van de zi'jplaanten. Ik hebbe ok zien laoten dat et niet uutmaekt of de zi'jplaante een grössoorte of een kruudachtige plaante is, of de plaante bekend staot as een soorte mit positieve of negatieve reaktie weeromme en of de zi'jplaante op vroeg of laete uut de perduktie neumen gröslaand staot. De risseltaoten gavven ok an dat wassende kelonisaosie van mycorrhizaschimmels verbaand hul mit et aachteruutgaon van de biomassa van zi'jplaanten. Bliekber kun mycorrhizanetwarken et ankommen van zi'jplaanten in gröslanen ofremmen in plaets van d'r bi'j te helpen.

Uut mien studie dot blieken dat veraanderings in de plaantegemienschop invloed hebben op de saemenstelling van mikroorgenismes en aolties in de bojem, en dat die veraanderings veurnaemelik tewegebrocht wodden deur de him wiezigende saemenstelling van anwezige eigenschoppen van plaanten, en niet veural deur et tal plaantesoorten. 



\section{Acknowledgements / Dankwoord}

Dear colleagues, you made TE a fantastic environment to work in! I continue to think that I am spoiled and that I will never find such a great atmosphere again.

Gerlinde, Wim en George, bedankt dat ik deze mooie positie heb gekregen. Ik had vooraf niet kunnen bedenken dat ik zoveel nieuwe vaardigheden zou gaan leren en zo'n fantastisch team aan begeleiders zou krijgen. Ik wil jullie bedanken voor jullie positieve instelling en de stimulerende werkomgeving. Ik kon het zeer waarderen dat jullie mij de vrijheid gaven om dingen zelf uit te zoeken. In het begin leidde dat tot lange dwalingen, maar ik leerde gaandeweg om focus te vinden. Jullie hebben alle drie een geheel andere schrijfstijl, dat was soms een uitdaging, maar ik heb daardoor ook geleerd om zó te schrijven dat het voor iedereen helder is ;-). Bedankt voor jullie begrip dat ik mijn promotieonderzoek tijdelijk op een laag pitje zette om bij mijn familie te kunnen zijn. Ook vond ik het erg fijn dat ik de ruimte kreeg om op de tuinderij te gaan werken, zodat ik naast het denk en schrijfwerk ook buiten met mijn handen kon werken.

Janneke, Freddy, Roel, Tanja, you helped me out a lot during practical work. I am glad that you always made time to explain me how things work and where to find the material I was looking for. Janneke, thank you for your time in helping me sorting out the right primers, to ease my mind when I thought I messed things up completely and for you humour that most of all. Henk Martens, thank you for teaching me the whole circus from soil sampling in Jena to sequencing the soil microbiomes. You are great company and a dedicated technician! Iris, thanks a lot for your help in the chemical lab and your patience to show me new techniques, we had a lot of fun! Henk Duyts, thank you for your time and help in identifying all nematode samples. Without your help, my promotion would have last much longer. Eke and Gregor, you are great help in and around the Fytotron. Thank you for keeping things organised Gregor! Eke, thank you for always being around to help and your creativity is outstanding! Roel and Gerard, thank you for the nice conversations and teaching me all I need to know about nematodes ;-).

Marta and Rutger, you are my PhD buddies! We shared so much fun, thoughts, frustrations, food, drinks, etc. I am very happy that you were always there. Marta, one day we will make a great biking tour, perhaps around your home place, which I still love to see. Thank you for making me feel always being welcome in your office for random or urgent questions, for chocolate and at moments of panic (yes, I panic too now and then!). Rutger, it was great 
to organise the writing weeks together, those were amongst the best weeks of my $\mathrm{PhD}$. Also traveling together to the ESA and our trip in Florida were amazing. I will never nearly accidentally feed you to an alligator again, promise! Thank you for teaching me so many new plant and bird species. I appreciate the many lunches, dinners and nice drinks. It was an honour to be your paranimph, and the 'regenwormen' game is one of the best presents I ever got, thanks buddy!

Stefan, thank you for all the advice you gave me on writing and analyses and for the many beers that you brought from Germany. You and Rutger taught me that beer (wine, whiskey) is a great remedy for writer's blocks. Casper, Jasper, Guusje, Robin, Tis en Heike bedankt voor jullie gezelligheid, vrolijkheid, mooie discussies en verhalen! Roeland, mijn held! Ik vond het tof dat je nog regelmatig even langskwam om bij te kletsen. Janna, bedankt voor de leuke en informatieve wandelingetjes! Mattias en Basten, bedankt voor het uitdoktoren van de pipelines, dat was zonder jullie nooit gelukt! Dedmer en Peter, et wie in eare om tegearre et Nederlânsk Ynstitút foar Ekology te fertsjintwurdigje! Ik sil jim tegearre misse ;-). Olga, thank you for your help with statistics and your encouragements, Emilia, thank you for the nice discussions and your regular show ups in the office to check how I was doing, and for your help and advice on sequence data clean up and analysis. Haikun, I enjoyed sharing the office with you, you work very hard and I admire your perseverance. And my previous office mates Ming Gang and Stijn, I missed the liveliness in our office when you left!

Katja and Maddy, it was already great to meet you via the Jena consortium and that the both of you are now in Wageningen is so much fun! Your kindness, fantastic food, advice, sharing of stories and frustrations all helped me to get through my $\mathrm{PhD}$. You are lovely people! Natalie, Julia, Jan, Lionel, Jordan, Thomas, and all other PhDs of our Jena Experiment generation, thank you for the great times we had in Jena, during the retreats and workshops but most of all on the field. I will never forget the summer that we all camped on the field site, some of you camped for weeks in a row. We all worked hard during the day and in the afternoon the field was our camping, we swam in the river, had lovely barbeques, had plenty of beers, and played the funniest games with the material that we found on the field. When the gardeners came back in the early morning, it was 'their' field again. Dear gardeners of the Jena Experiment, thank you for keeping the field so good and thank you for your patience while explaining how to identify the different grass and clover species over and over again. I also like to apologise for the time that I wrote 'sternschnuppe' with yellow paint on the purple field truck. I thought it would wash away 
with the rain, but the rain did not come in time. Cameron and Anne, thank you for keeping things organised on the field and your great help in negotiating between $\mathrm{PhDs}$, gardeners and PIs. It was great to work with you and I enjoyed the times that we discussed about science and shared drinks and food.

Sophia, Terhi, Jacqueline and the rest of Bernhard's group, I am very happy that I got to know you. We met several times at fantastic locations. You are my science travel buddies! The time we spend together in Florida during the ESA is a great memory. Sophia, a special thanks for your friendship and hospitality, and one day you will be a professor! Bernhard, the writing week in the Swiss Alps was wonderful, it was a pleasure to join and nice to be part of another science group for two weeks.

Natalie, you are a fantastic colleague. We shared a lot of time together during practical work in Jena and in Wageningen. Thank you for helping me out so much with the initiation of the Veluwe experiment. It was great to work together and there is still some work to do for the near future :-). I am glad that you know how to keep things organised. I enjoyed our discussion about science and life and I love the beautiful postcards that you send and the fantastic cakes that you made, thank you so much!

I would like to thank all the members of the secret Van Der Putten Plein Organisation. It was an honour to be your secretary and I wish you happy sessions for the future. Let the legend continue!

Lennart, Claudius en de rest van het PE\&RC-team, hartelijk dank voor de fantastische ondersteuning en alle mooie activiteiten die jullie organiseren. Jullie bijdrage is onmisbaar!

Anneke, Jorien, Christoph, Saskia, Joost, Jorrit (nogmaals dank voor je hulp in het lab!), Johan, Jornt, Casper, Noël, Linde, Folmer en alle andere vrienden, bedankt voor alle gezelligheid, etentjes, biertjes, geklets, feestjes, fiets-, klim-, langlauf- en skitochten, kampeerweekenden, avondjes in het park, aan de Rijn, in de sauna, adventure races, bordspellen, curling! En alle andere mooie momenten. Jullie zijn fantastische vrienden, bedankt voor al het goeds dat ik van jullie gekregen heb!

Homies! Jochem, Paula, Maite, Miguel, Lucia, Airen, Marlou, Robertooo! And all others former housemates of Huize Kakelbont. It was a fantastic time together, thank you for all the moments (coffee, pancakes, movies, chats, dinners, breakfasts, drinks, puzzles, walks, parties, etc.) it was great to share our lives together. Thank you for taking good care of me! 
Ik wil de Stichting Stellingwarver Skieversronte, mit naeme Henk Bloemhoff, hattelik bedaanken veur hun hulp mit de vertaeling van de saemenvatting van mien proefschrift naor et Stellingwarfs. Ik bin d'r slim bliede mit!

Ik ben dol op trekker rijden. Als ik van het land van Tuinderij de Stroom terugreed naar boerderij De Lingehof en op het asfalt de klei uit de banden vloog dan had ik een glimlach van oor tot oor. Het werk op de tuinderij gaf mij veel voldoening: buiten aan het werk zijn met zaaien, planten, oogsten, wieden, wieden, wieden, watergeven, bestellingen klaarmaken en bij klanten afleveren. Ik krijg alleen maar meer zin om een eigen tuinderij te beginnen! Ik heb genoten van de prachtige verhalen van de vrijwilligers, de goede gesprekken, het uitwisselen van kennis over landbouw, de heerlijke taarten en cakes en vooral van het vele lachen samen. Linde, Welmoet, Angelien, Jechina, Dorien, Mark, Ben, Ton, Paulien, ...(oei, ik vergeet vast een paar mensen) ..., én Petra, André, Peter en Twan, bedankt voor de mooie tijd!

Ik geniet altijd van de dagjes en weekenden in Boyl, dan ben ik er even helemaal uit. De gastvrijheid, de prachtige omgeving en de ruimte om te doen en laten waar ik zin in heb zijn voor mij ultieme vrijheid en ideaal om weer even op te laden voor het denk- en schrijfwerk in Wageningen. Bij thuiskomst wordt meestal direct een lijstje met klusjes en activiteiten opgesteld om mij bezig te houden. Ik ontsnap altijd graag even naar de buren, familie Kleinhoven, voor de koffie, koeien kijken en jonge katjes aaien :-). Ik vind het heerlijk om bij jullie te zijn. Ik heb nog altijd het idee dat als ik bij jullie binnenloop, een boterham smeer en die op de bank opeet, dat jullie je daar niet over zullen verbazen. Ik heb er altijd van genoten dat ik van kleins af aan bij jullie op de boerderij mee mocht helpen en dat heeft zeker invloed gehad op mijn interesse voor landbouw en milieu. Wim, Albert, Jeannette, Martine, Ruben en in gedachten Alie, jullie zijn de beste buren die ik mij kan wensen.

Niels, Jorne \& Mary, jullie zijn mijn fantastische broers en schoonzus. Jullie hebben de afgelopen jaren goed voor mij gezorgd :-). Samen met jullie op stap en samen aan het werk waren heerlijke afwisselingen met het promotiewerk. Niels, bedankt voor alle ritjes achter op de motor en de stiekeme motorrijlessen. We hebben heel wat uren samen in de tuin gewerkt, door het bos gesjouwd, samen een voederakkertje aangelegd, dingen geklust (dingen doen met spullen), en samen prakkeseren. En nogmaals dank voor alle fantastische verjaardagscadeaus! Je hebt mij ook leren motorzagen en de dagen samen met de familie in het bos zagen waren heerlijk, 's avonds bekaf en languit op de bank! Jorne, bedankt dat jij 
je kleine zusje meenam naar de bodem van de Noordzee. De weekendjes wrakduiken waren hele mooie avonturen. Het is zó gaaf om langzaam af te dalen langs de ankerlijn, de donkere en ruige diepte in, en dan opeens doemt daar een scheepswrak op, omringt door zeeleven. Mary, bedankt voor jouw heerlijke kookkunsten en de keren dat we samen gingen snuffelen in tweedehandswinkels. Ik vond het heel erg mooi dat ik met jou mee mocht naar de Filipijnen en jouw familie daar heb leren kennen :-). Binnenkort woon ik weer wat dichter bij het nest en ik kijk ernaar uit om jou en mijn broers weer vaker te zien.

Sander, wij zijn slechts $5.62 \mathrm{~km}$ bij elkaar vandaan opgegroeid, maar kwamen elkaar pas dit jaar tegen. Ik ben dolblij dat wij elkaar gevonden hebben en in de korte tijd dat we elkaar kennen hebben we al heel veel samen ondernomen. Ik waardeer jouw positiviteit en doorzettingsvermogen en ben heel nieuwsgierig naar onze toekomst :-) Lydia, Jan, Mark, Anke, Amarinske en Heico, jullie zijn lieverds!

Lieve pa en ma, ik wil jullie enorm bedanken, niet alleen zozeer voor de jaren van mijn promotieonderzoek, maar voor alle jaren. Jullie hebben mij grotendeels gevormd tot wie ik ben en ik heb van jullie geleerd om door te zetten, positief te blijven, te relativeren en te genieten van de kleine dingen in het leven. Die eigenschappen en jullie onvoorwaardelijke steun hebben mij geholpen tijdens het promotieonderzoek, maar ook tijdens de studiejaren daarvoor. Ik besefte tijdens mijn jaren in Wageningen dat het een voorrecht is geweest om op te groeien met zoveel ruimte en natuur om mij heen, dat jullie mij en mijn broers het vertrouwen gaven om in het bos te spelen, dat wij huisdieren mochten hebben om voor te zorgen, dat wij soms mee moesten helpen in de tuin en mee mochten op jacht. Pa, de periode dat jij in het ziekenhuis lag was een moeilijke tijd, ik hoop dat jij er nog lang zult zijn, want ik kan nog veel van jou leren. Jouw schuur vol gereedschap en machines om hout mee te bewerken en om aan auto's, motoren en trekkers te sleutelen is een heerlijke plek om samen te klussen. Ook vind ik het prachtig dat wij nu samen op jacht kunnen, door de velden kunnen struinen met de groep van Boyl, of te gast mee in Boschoord en Vledder. Ma, jouw zorgzaamheid en jouw kijk op het leven zijn prachtig. Jij leerde mij dat je niet rijk hoeft te zijn om je rijk te voelen, wat jij en pa opgebouwd hebben is voor mij een paradijsje.

\section{Sigrid}

September 2018 


\section{About the author}

Sigrid Dassen was born in Boyl on the $29^{\text {th }}$ of October, 1985. She grew up in the beautifull surroundings of 'het Drents-Friese Wold'. Being raised nearly as much as by the neighbour on their farm as by her own parents the interest for nature, farming, and

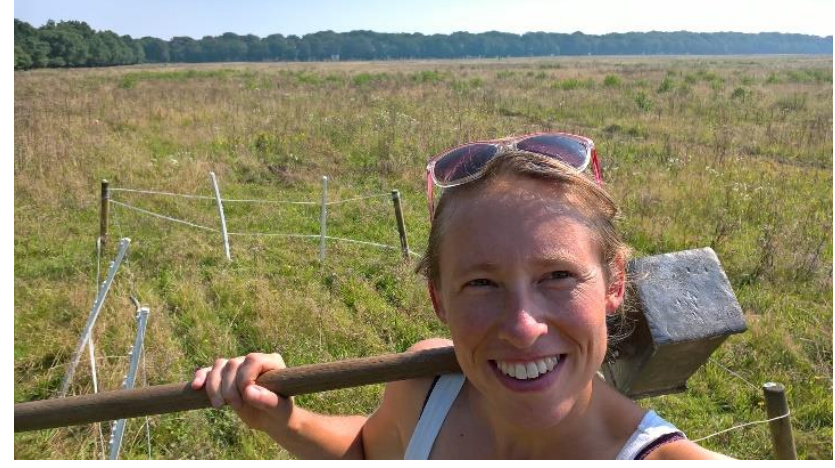
outdoors came naturally. At the Van Hall Institute in Leeuwarden she did a BSc in biotechnology and specialised in plant biotechonolgy. For her BSc she did an intership at the Institute of Biological, Environmental and Rural Science (IBERS) in Wales and another internship at Genetwister in Wageningen. For her BSc-thesis she worked on the detection, multiplication and identification of soil bound phages against plant pathogens, which was performed at Plant Reseach International, Wageningen. She continued her studies at Wageningen University with a MSc in Plant Sciences and specialised in plant pathology and entomology. During her MSc-internship at Crop System Analysis she performed germination studies on the plant parasitic weed Rhamphicarpa fistulosa and studied the interaction with the host plant rice. Her MSc-thesis was performed at the Plant Physiology group where she studied the chemical composition and ecological aspects of cucumber flower volatiles. Soon after her MSc graduation Sigrid started a $\mathrm{PhD}$ project under supervision of Wim van der Putten, Gerlinde De Deyn and George Kowalchuk on soil microbial and nematode communities and plant-soil feedback. Beside her PhD, Sigrid works on a organic farm and is involved in nature education. 


\section{List of publications}

Dassen S., Cortois R., Martens H., de Hollander M., Kowalchuk G.A., van der Putten W.H., De Deyn G.B. (2017) Differential responses of soil bacteria, fungi, archaea and protists to plant species richness and plant functional group identity. Molecular Ecology, 26, 4085-4098.

\section{Submitted and in preparation}

Dassen S., van der Putten W.H., De Deyn G.B. (in review in New Phytologist) Mycorrhizal networks in restoration grasslands suppress seedling biomass.

Musa Bandowe B.A., Leimer S., Meusel H., Velescu A., Dassen S., Hoffmann T., Oelmann Y., Wilcke W. (in review in Soil Biology and Biochemistry) Plant diversity enhances the natural attenuation of polycyclic aromatic compounds (PAHs and oxygenated PAHs) in grassland soils.

Dassen S., Hannula S.E., Kowalchuk G.A. Oram N.J., Ebeling A., Wagg C., van der Putten W.H., De Deyn G.B (in preparation) Plant traits drive root-associated fungal community composition more than plant species richness.

Dassen S., De Deyn G.B., Kowalchuk G.A. Duyts H., Hannula E.S., Snoek B., Ebeling A., Wagg C., van der Putten W.H. (in preparation) Plant trait effects on richness and abundance in the soil food web. 


\section{PE\&RC training and Education Statement}

With the training and education activities listed below the $\mathrm{PhD}$ candidate has complied with the requirements set by the C.T. de Wit Graduate School for Production Ecology and Resource Conservation (PE\&RC) which comprises of a minimum total of 32 ECTS $(=22$ weeks of activities)

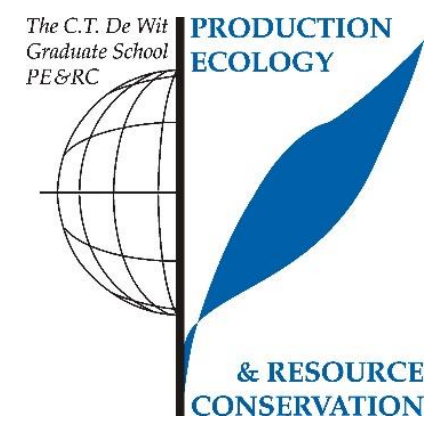

\section{Review of literature (6 ECTS)}

- Plant-soil feedback and diversity-productivity relationships (2014)

\section{Writing of project proposal (4.5 ECTS)}

- Plant-soil feedback and diversity-productivity relationships (2014)

\section{Post-graduate courses (5.6 ECTS)}

- $\quad$ Introduction into R; PE\&RC (2013)

- Nematode identification course; NIOO (2015)

- $\quad$ Soil Ecology; PE\&RC (2016)

\section{Invited review of (unpublished) journal manuscript (7 ECTS)}

- Molecular Ecology: root endophyte communities in dune grasses (2017)

- New Phytologist: soil microbial communities of tree species (2017)

- Agriculture, Ecosystems and Environment: N-fertilization effects on AMF community composition (2017)

- Frontiers in Microbiology: plant-plant competition mediated by AMF (2017)

- Plos One: land abandonment effects on plant community composition (2017)

- Molecular Ecology: climate change effects on soil processes (2017)

- Molecular Ecology: bacterial and fungal soil and plant endophyte community composition (2018)

\section{Competence strengthening / skills courses (5.3 ECTS)}

- $\quad$ Voice matters (2013)

- $\quad$ Reviewing a scientific paper (2014) 
- Competence assessment (2014)

- $\quad$ Techniques for Writing and Presenting a Scientific Paper (TWP) (2014)

- $\quad$ Project and time management (2014)

- Courses organised by the Jena PhD's (writing, analysis in $\mathrm{R}$, presentation skills) (2014-2016)

PE\&RC Annual meetings, seminars and the PE\&RC weekend (2.1 ECTS)

- $\quad$ PE\&RC Day $(2013,2015,2016)$

- $\quad$ PE\&RC Midterm weekend (2015)

- $\quad$ PE\&RC Last years weekend (2017)

Discussion groups / local seminars / other scientific meetings (7.2 ECTS)

- $\quad$ Mini symposium Writing a world class paper (2013)

- $\quad$ Digging deeper UK, Londen (2013)

- $\quad$ Organisation of the Jena PhD workshops and meetings (2013-2015)

- $\quad$ WEES (2014)

- Vegetation - Soil interaction symposium (2014)

- $\quad$ Plant-soil interactions (2016-2017)

- $\quad$ Frontiers in Ecology (2017)

International symposia, workshops and conferences (17.1 ECTS)

- $\quad$ Rhizosphere 4; Maastricht (2014)

- Global soil biodiversity conference; Dijon France (2014)

- $\quad$ NERN, NAEM; Lunteren (2014-2017)

- $\quad$ BES; Liverpool (2016)

- $\quad$ ESA; Florida (2016)

- $\quad$ ICOM9; Prague (2017)

\section{Lecturing / Supervision of practicals / tutorials (2.1 ECTS)}

- Ecological aspects of bio-interactions, supervision of practical

- Presentation about arthropod diversity in the Jena Experiment for the WUR biodiversity course 


\section{Colophon}

The research presented in this thesis was conducted at the Department of Terrestrial Ecology at the Netherlands Institute of Ecology (NIOO-KNAW) in Wageningen.

This is NIOO thesis 158

The research in this thesis was funded by the Dutch Organisation for Scientific Research (NWO) and the German Research Foundation (DFG).

Text, figures and photos: Sigrid Dassen

Cover design and layout: Sigrid Dassen

Printed by: Digiforce || Proefschriftenmaken 



\title{
Propositions
}

1. Decreasing plant species richness affects microbial richness and abundance by reducing variation in plant morphological and developmental traits.

(this thesis)

2. Changes in plant community trait composition affect the base of the green pathway of the soil food web more than the brown pathway.

(this thesis)

3. The governmentally imposed practice of liquid manure injection is disastrous for soil life, insects and farmland birds.

4. Wild animals benefit more from improved breeding areas than from hunting bans.

5. Teenager and young adult depressions will be reduced by teaching children not to measure their own happiness against the happiness of others.

6. Banning pesticides, pavements and gravel from private gardens will inspire children's creativity and passion for nature.

Propositions belonging to the thesis entitled:

\section{Diversity relations of plants and soil microbes}

\author{
Sigrid Dassen
}

Wageningen, 29 October 2018 